Assessment of Effectiveness of

Geologic Isolation Systems

\title{
Relationships of Dispersive Mass Transport and Stochastic Convective Flow Through Hydrologic Systems
}

C. S. Simmons

January 1981

Prepared for the

Office of Nuclear Waste Isolation

Under its Contract with the

U.S. Department of Energy

Pacific Northwest Laboratory

Operated for the U.S. Department of Energy

by Battelle Memorial Institute 
This report was prepared as an account of work sponsored by the United States Government. Neither the United States nor the Department of Energy, nor any of their employees. nor any of their contractors, subcontractors. or their employees, makes any warranty, express or implied, or assumes any legal liability or responsibility for the accuracy, completeness or usefulness of any information, apparatus. product or process disclosed, or represents that its use would not intringe privately owned rights.

The views, opinions and conclusions contained in this report are those of the contractor and do not necessarify represent those of the United States Government or the United States Department of Energy.

\author{
PACIFIC NORTHWEST LABORATORY \\ operated by \\ BATTELLE \\ for the \\ UNITED STATES DEPARTMENT OF ENERCY \\ Under Contract DE-AC06-76RLO 1830
}
Printed in the United States of America Available from
National Technical Intormation Service United States Department of Commerce 5285 Port Royal Road Springfield. Virginia 22151

Price: Printed Copy 5 $\therefore$ Microliche $\$ 3,00$

$\begin{array}{cc}\text {-Pages } & \begin{array}{c}\text { NTIS } \\ \text { Selling Price }\end{array} \\ 001-025 & \$ 4.00 \\ 026-050 & \$ 4.50 \\ 051-075 & \$ 5.75 \\ 076-100 & \$ 6.00 \\ 101-125 & 56.50 \\ 126-150 & 57.25 \\ 151-175 & \$ 8.00 \\ 176-200 & 59.00 \\ 201-225 & \$ 9.25 \\ 226-250 & \$ 9.50 \\ 251-275 & \$ 10.75 \\ 276-300 & \$ 11.00\end{array}$




\section{5}

RELATIONSHIPS OF DISPERSIVE MASS TRANSPORT AND STOCHASTIC CONVECTIVE FLOW THROUGH HYDROLOGIC SYSTEMS

C. S. Simmons

January 1981

Prepared for the Office of Nuclear Waste Isolation under its Contract with the U.S. Department of Energy

Pacific Northwest Laboratory

Richland, Washington 99352 


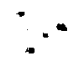

$\because$

?

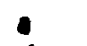

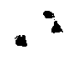




\section{PREFACE}

The objectives of the Transport Methodology Development tasks with in the Assessment of Effectiveness of Geologic Isolation Systems (AEGIS) Program include studies into the best theoretical description of contaminant transport under uncertainty. Uncertainty in water flow velocity appears to be a major factor in determining the magnitude of contaminant dispersion expected in a ground water system. This report discusses some concepts and mathematical methods relating dispersive contaminant transport to stochastic aspects of ground water flow. The theory developed here should not be construed as absolutely rigorous mathematics, but is presented with the intention of clarifying the physical concepts. However, it is hoped that theory can provide a basis for tractable computational methods. 


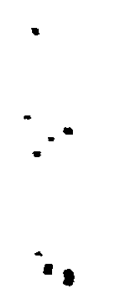

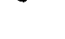

: 
Present research into fluid flow technology indicates that the classical convective-dispersion equation description of mass transport in a permeable media at a macroscopic scale should be replaced by a more general stochastic flow field methodology. The dispersion component of transport in an aquifer is best characterized at the greater megascopic scale by variations or uncertainties in the flow velocity. Typically, laboratory measurements of the disperison coefficient (or dispersivity) from core samples do not account for the considerably greater dispersion observed in field experiments. Hence, predictions of contaminant transport in a ground water system, based on mean flow velocities and laboratory-measured dispersion parameters, will be unreliable.

In some cases, an effective dispersion coefficient relevant to an aquifer's length scale can be establishd by solving an inverse fitting problem for a tracer field experiment. However, such a coefficient may characterize only the orignal region of the tracer measurements and does not necessarily apply beyond that region. Moreover, the convective-dispersion equation used as a mathematical model, based on Fick's Law, may not correctly describe the concentration distribution as a consequence of uncertainty in velocity. Indeed, vast contaminant travel times through an actual aquifer often eliminate the possibility of a direct field experiment.

Various mathematical formulations of transport founded on a stochastic convective flow approach are reviewed. All proposed methods connect the expectation and variance of solute concentration with a stochastic description of flow velocity. The foundation of those methods is an ensemble viewpoint of a ground water system. That is, any one sampling or simulation of transport represents only one member of an ensemble of possible transport predictions. The "true" results are realized only as an average over the ensemble. Perturbation methods, utilizing only mean and spatial covariance of velocity, are an effective approach if the velocity variation is small relative to its mean. Methodology of geostatistics (Kriging) can be applied to obtain the required statistical description of spatial variability in hydraulic properties causing uncertainty in velocity. A direct velocity ensemble approach can treat 
arbitrarily large velocity variation, but is limited in application because the complete velocity field probability distribution is seldom known. Random flow tube models of transport are an exception, since the ensemble is obtained directly from a ground water system as the streamlines. Such models have a simple representation in terms of a solute travel time distribution associated with the velocity ensemble.

Random motion models similar to random walk methods also show promise. In these models, the transport can be simulated from a conceptualized movement process based on the statistical properties of the velocity uncertainty observed at a relevant length scale.

The main conclusion deduced from stochastic models is that a considerable restructuring of present computer simulation models for contaminant transport is required in order to describe properly dispersion effects over an entire ground water system. 


\section{CONTENTS}

SUMMARY

I. INTRODUCTION

Concepts of Hydrodynamic Dispersion

Difficulties with the Convective-Dispersion Equation . $\quad$ • $\quad 2$

Relationship of Dispersion and Uncertainty . . . . . . 5

The Ensemble Concept . . . . . . . . . . 8

Objectives and Limitations . . . . . . . . . . . . 10

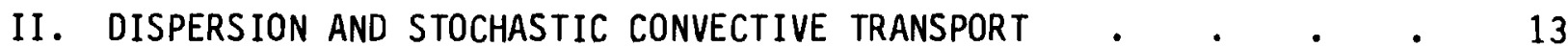

A. Ensemble Methods . . . . . . . . . . 13

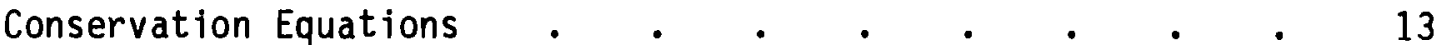

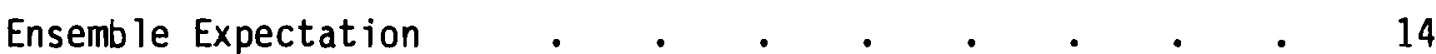

Solutions of Convective Transport Equation $\quad$ - . . . . 17

Initial value problems $\quad . \quad$. $\quad . \quad \ldots \quad . \quad$. 17

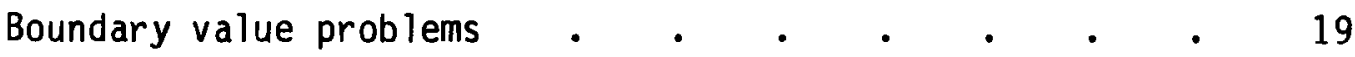

Higher dimensional solutions $\quad . \quad$ e $\quad . \quad$. $\quad . \quad$. 19

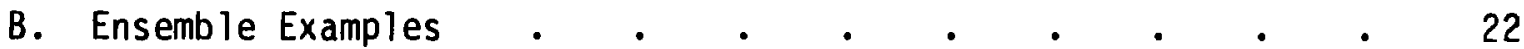

1. Constant Velocity Ensemble . . . . . 22

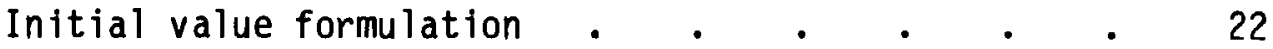

Boundary value formulation . . . . . . $\quad$. 25

Conservative convective flux . $\quad$. $\quad$. $\quad$. 34

2. Spatially Variable Ensembles . . . . . . 38

3. Purely Random, Stationary, Gaussian Processes Ensembles . 42

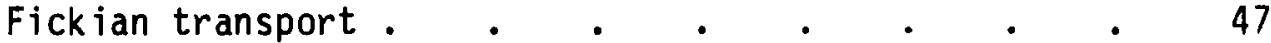


Ensemble Statistical Moments . . . . . . . 48

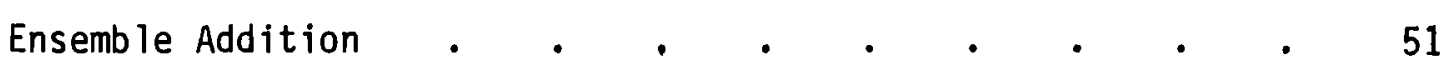

C. Travel Time Ensembles . . . . . . . . . . . . . 54

Definition . . . . . . . . . . . 54

Boundary Condition Formulation . . . . . . . 56

Moment Generating Functions . • • • • • 59

Initial Condition Formulation . $\quad . \quad$. $\quad$. 64

Connection between travel time and distance . . . 68

Spatially stationary ensembles . $\quad$ - . . . . . 71

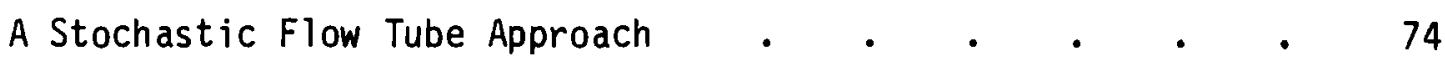

Non-uniformly distributed release $\quad$ - $\quad$. $\quad$. 77

Deterministic simulations . . . . . . . . 78

Observation of a travel time p.d.f. $\quad . \quad$. $\quad . \quad$. 79

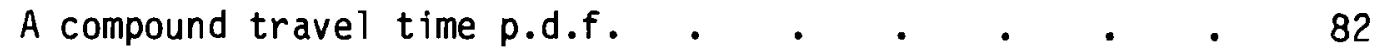

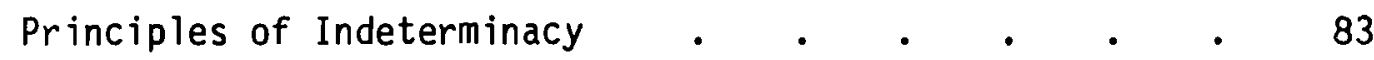

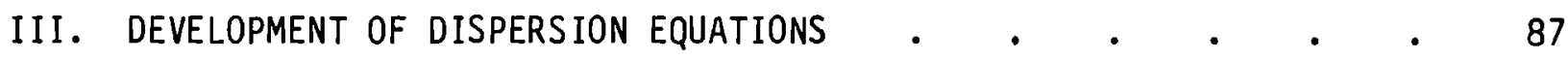

General Results . . . . . . . . . . . 87

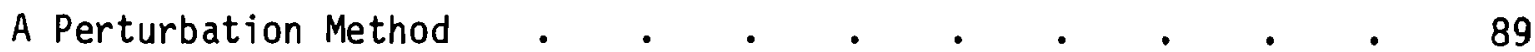

Constant Mean Velocity Cases . . . . . . . . . 92

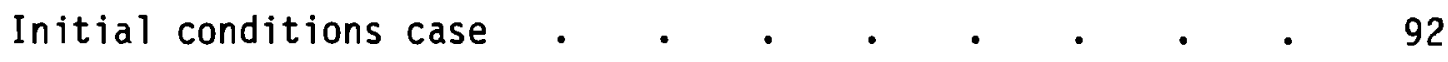

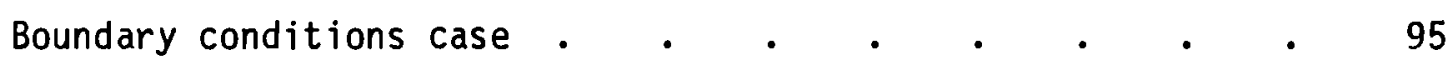

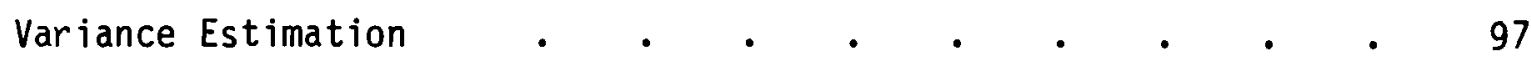

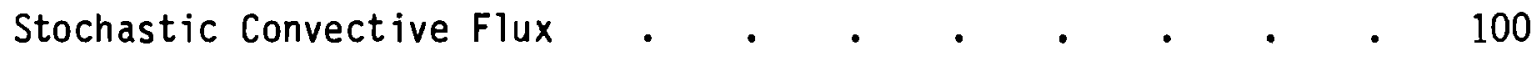

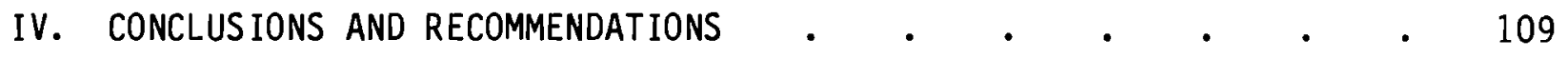

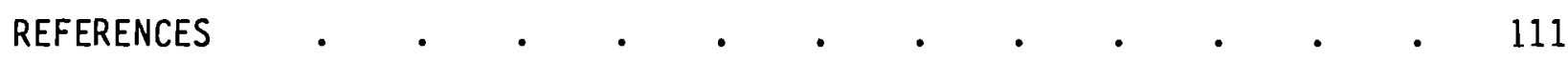




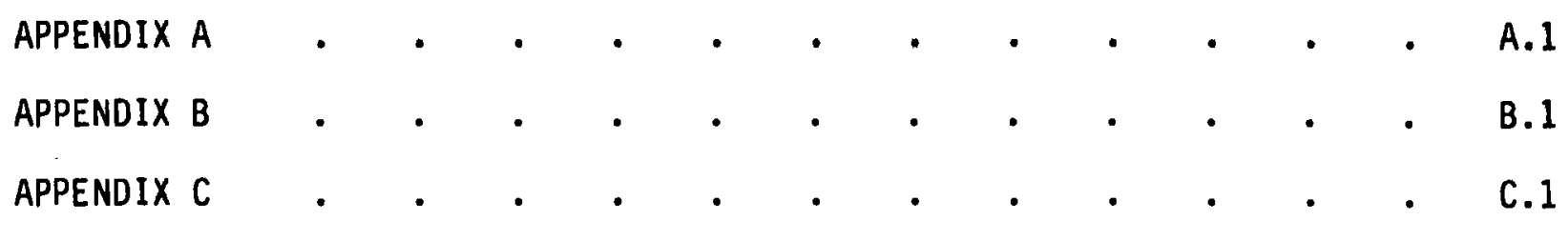
1

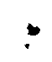

$\because$

$+$ 


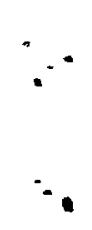

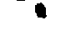

•

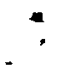

1 


\section{INTRODUCTION}

\section{CONCEPTS OF HYDRODYNAMIC DISPERSION}

Dispersion is an important physical phenomenon that must be taken into account when evaluating the environmental consequences of transport of a hazardous substance by a ground water system. A helpful aspect of dispersion is its reduction of possible peak contaminant concentrations arriving at the biosphere. However, detrimental aspects are that a contaminant will be dispersed over larger regions and will have earlier arrival into the environment than predicted by mean ground-water velocities alone.

In fluid flow through porous media, dispersion refers to the spreading of a dissolved substance, a solute. As a solute is transported by a flowing ground water system, it spreads occupying regions greater than would be expected based on fluid convection alone. This spreading, that is not accounted for by convection, is called hydrodynamic dispersion and is caused by two mechanisms: molecular diffusion and mechanical dispersion. Diffusion results from the migration of solute from nigher to lower concentration regions as a consequence of molecular thermal motion, and it occurs irrespective of fluid flow. Mechanical dispersion results from the varied pathways traveled by solute molecules as they are transported through the pore channels of a porous medium. The varied pathways are a consequence of flow velocity variations within and between pore channels. Mechanical dispersion thus depends on the local ground water velocity, which represents the average of flow velocities through pore channels. In an aquifer, mechanical dispersion is usually the dominant of the two mechanisms.

It is well known that estimation of solute concentrations depend on the sample volume size, or what is called the length scale of a measurement. In a similar way, the quantification of dispersion depends on the measurement scale. A sample volume, which is small relative to the pore and grain size of a medium, determines measurement at a microscopic scale. When a microscopic sample volume is changed in location, the concentration within can display considerable variation. The macroscopic scale is attained at a particular location when the sample volume is sufficiently large enough that variations of concentration within the pore system are not detected as the sample volume 
is further increased. The minimum volume for macroscopic measurement of a property is called the representative elementary volume (Bear 1972). Local measurements of hydraulic properties, such as porosity and hydraulic conductivity, are determined at this macroscopic scale. In an actual aquifer, there is an upper limit on the macroscopic sample volume that represents local hydraulic properties. Beyond that limit a sample volume will encompass different stratigraphic units or medium heterogeneities composed of varying morphology (sand, silt, clay, gravel, stone, etc.). This domain beyond the limit of macroscopic measurement is the megascopic scale, and represents global measurement. It is apparent from the nature of ground water systems that the three scale categories cannot be defined independently of location and time. That is, there is no assurance that a particular sample volume used to measure hydraulic properties will remain in a fixed scale category as location and time change.

Observations of dispersion effects will depend on the measurement scale as demonstrated in field tracer experiments (Pickens et al 1978). Indeed, dispersion at the microscale is caused by diffusion, whereas macroscopic dispersion is caused by mechanical dispersion. Megascopic dispersion effects will appear as a consequence of fluid flow variations at the macroscopic level. The common principle governing dispersion at each scale category is that it results from flow velocity variation in the categories of smaller length scale.

Difficulties With the Convective-Dispersion Equation

Historically, the classical convective-diffusion equation, based on Fick's Law for diffusion flux, was extended to describe transport in porous media by including dependence on a tensorial dispersion paramter, $\overleftrightarrow{D}$, which is a function of the mean local flow velocity. The so-called convectivedispersion transport equation obtained is a phenomenological law verified in statistically homogeneous, granular media, for controlled laboratory experiments which are usually done in soil columns. (See J. J. Fried 1975 for a complete review of the present theory of dispersion.) The genera! macroscopic transport equation for solute in a partially-saturated porous medium is: 


$$
\frac{\partial}{\partial t}(\phi c)+\nabla \cdot(\phi c \vec{v})=\nabla \cdot(\phi \vec{b} \cdot \nabla c)
$$

where

$c=$ concentration of solute in solution on the macroscopic scale

$\phi=$ volumetric water content of the porous medium

$\vec{v}=$ Darcy water velocity

$\overleftrightarrow{\mathrm{D}}=$ solute dispersion tensor.

The convective flux is:

$$
\vec{J}_{c}=\phi c \vec{v}
$$

and generalized Fickian flux is:

$$
\vec{J}_{c}=\stackrel{\leftrightarrow}{b \cdot} \cdot \nabla c
$$

The measure of a porous medium's capability for producing mechanical dispersion is called dispersivity. Diffusivity is the corresponding parameter for diffusion. Dispersivity can be resolved into two principle components: one in the direction of local ground water velocity called longitudinal dispersivity, and the other perpendicular to the velocity called transverse dispersivity. According to theory, the longitudinal and transverse dispersion coeficients are related to dispersivity a by:

$$
\begin{aligned}
& D_{L}=D_{m}+\alpha_{L} \mid \vec{v} \mathbf{I}^{n} \\
& \left.D_{T}=D_{m}+\alpha_{T} \mid \vec{v}\right\}^{n}
\end{aligned}
$$

where $L$ and $T$ denote longitudinal and transverse components, respectively

$$
\begin{aligned}
D_{m} & =\text { the molecular diffusivity } \\
n & =\text { an exponent usually equal to one. }
\end{aligned}
$$

The dispersivities are supposed to represent a fundamental property of the porous medium. Laboratory and field experiments, however, indicate that the 
dispersivity increases with an increase in length scale of the measurement (Pickens et al 1978).

Laboratory column experiments yield dispersivity values determined from breakthrough curves, usually of the order of $10^{-2}$ to $1 \mathrm{~cm}$, whereas calibration of computer models to field experiments yield values in the range of 10 to $10^{2} \mathrm{~m}$ (Pickens et al 1978; Fried 1975). This discrepancy in dispersivity values indicates that dispersion in a field experiment is considerably greater than would be predicted by a laboratory measured dispersivity. Detailed field sampling experiments demonstrate that the greater field measured dispersivities can be attributed to undetected macroscopic variations in ground water flow velocity. An intensive field experiment performed by Biggar and Nielsen (1976) showed that dispersivity. exhibits considerable spatial variability, even over a small region. Their measurements indicate that using only a few sampled dispersivity values would fail to describe dispersion in the experimental region. Indeed, the concept of a fundamental dispersivity in Eq. 4 seems to be contradicted.

A theoretical inconsistency in Eq. 4 has been pointed out, which would imply that under certain initial conditions solute would move counter to the flow velocity direction (Simpson 1979). In the absence of diffusion, it is physically impossible for mechanical dispersion to produce upstream movement of solute under the restriction of occurrence of only laminar flow through porous media at the macro-scale. The validity of Equations 1 and 4 applied to transport in fracture rock has also been questioned. Those limitations, however, will not be discussed here.

Difficulties with the transport Eq. 1, outlined so far, are caused by lack of correspondence between measurement at megascopic scale and the assumption that velocity, porosity, and dispersivity (transport parameters), are deter-ministic at the macroscopic scale. In principle, transport described by Eq. 1 could be treated as a deterministic problem provided the required parameters could be measured in complete detail at the macroscopic scale. The impracticality of sampling an entire ground water system at macro-scale, however, requires dealing with megascopically averaged transport parameters. Direct consideration of Eq. 1 at the megascopic level required evaluation of a stochastic transport description, as a natural consequence of 
the inherent uncertainty in transport parameters. Uncertainty will stem from two sources: the incomplete description of transport parameters and instrument measurement error. Thus, a method of averaging the convective-dispersion equation appears to be needed. Such an approach requires, as a fundamental assumption, that Eq. 1 remains valid at the megascopic scale.

A recent critical review of foundation theories of solute transport in porous media indicates that, at present, there is no physically meaningful and mathematically rigorous method for deriving the macroscopic dispersion coefficient applicable in Fick's Law (Eq. 3) (Sposito et al. 1979). That is, present concepts do not yield a dispersion coefficient based on microscopic dynamics and media properties that is valid over macroscopic regions.

Derivations of Eq. 1, based on entirely kinematic discussions such as fluid mechanics, random walk, and statistical mechanics, do exist, but they all include assumptions that cannot be given physical justification. The conclusions drawn from the review is that the validity of Eq. 1 at the megascopic scale is unknonn. And the implication is that a dispersion coefficient measured in a small region with transport occurring over a short period is not proven valid when extended to a larger region. It therefore appears that the only certain megascopically valid transport equation is the statement of solute conservation, or convection equation alone.

Relationship of Dispersion and Uncertainty

Recent literature describing field experiments and stochastic analys is of mass transport at the global scale of an aquifer has demonstrated that dispersion caused by variations in local flow velocity is often many orders of magnitude greater than local hydrodynamic dispersion (Pickens et a1. 1978, Simpson 1979, Tang and Pinder 1979). Tang and Pinder (1979) studied the effect on the convective-dispersion equation of treating the transport parameters (velocity and dispersivity) as macroscopically uncertain (Tang and Pinder 1979). The approaches taken by various authors are not only different in mathematical method but are also different in viewpoint as regards the origin of randomness in transport parameters. Gelhar et al. (1979) view uncertainty as resulting from hydraulic conductivity variations associated with the inherent spatial variability of a stratified heterogeneous aquifer. 
On the other hand, Tang and Pinder (1979) view uncertainty as due to error in measurement and interpretation of field experiments. The common viewpoint is that global mass transport is a stochastic process, no matter what the origin of randomness. Flow velocity is the major random transport parameter.

In view of the concept that a ground water system in steady state conditions has a uniquely determined flow velocity at each location and, therefore, constitutes a perfectly deterministic system, perhaps the main question is why must the transport in such systems be treated as stochastic. A layered aquifer provides an example flow system having a megascopic velocity uncertainty if only the mean velocity over its layers is known. Although the detailed flow field is deterministic, the observed uncertainty in velocity constitutes a stochastic velocity. For example, a tracer distributed over the layered profile would display a concentration front location described by the mean velocity only if the unknown variation is sufficiently small. The detailed location of a moving tracer front would, in general, display a finger concentration distribution corresponding to the velocity variation over layers. An observation well fully penetrating and sampling the aquifer would reveal only an average concentration over the fingered front. That concentration described by a breakthrough curve as a function of time could possibly indicate dispersion considerably greater than that predicted by the mean velocity and a local dispersion coefficient. A point release of tracer might have its transport described by an effective dispersion coefficient if the exchange between layers were sufficiently random so that the solute distribution over the aquifer profile became Gaussian. In that case the aquifer sampled breakthrough curve could be described by the mean velocity and just one additional parameter related to the velocity's variance. An infinity of velocity distributions and configurations of layers could conceivably produce the same breakthrough curve observed at the well. Although the details of the finger fronts would differ, the same mean velocity and effective dispsersion coefficient would characterize the breakthrough. In this way, the dispersion observed for that infinity of equivalent flow systems is characterized by the original uncertainty as expressed by variance in velocity. Note that the foregoing discussion assumes that velocity is 
constant along the length of each layer so that variance suffices to describe the random variation over layers. In general, a covariance function is required to characterize the random variation. Marle (Fried 1975) has demonstrated this result that a layered system with sufficiently large transverse dispersion causing mixing of solute between layers can be described by Eq. 1 with an effective longitudinal dispersion coefficient (Fried 1975). This is a case for which the transport remains Fickian that is, Eq. 3 holds at the megascopic scale. In contrast, layered systems allowing negligible exchange of solute can exhibit non-Fickian transport when concentration is measured over an aquifer profile (Simpson 1979). That is, the dispersion flux, which is not accounted for by convective flux as the product of mean velocity and concentration, may not be proportional to the concentration gradient. Regardless of whether or not Fick's law governs dispersive transport in a layered aquifer, dispersion perceived on a megascopic scale is mainly due to the stochastic convective transport occurring over layers. It appears that the uncertainty of flow in our hypothetical layered aquifer could be resolved by simply obtaining a complete description of layers from core samples. This approach, however, will generally fail because, as observed in field experiments, regions appearing homogeneous in geological classification will nevertheless manifest velocity variation.

Now a layered ground water system is not a unique example manifesting the indicated stochastic convective dispersion. Indeed, all ground water computer simulation models utilize a discreatized description of a region and its flow parameters, and the uncertainty in calculated velocity between the grid point network (nodal system or finite elements) is not accounted for in a transport analysis. Again, a system with an incomplete description of velocity variation over regions can result in estimating less dispersion than found in a real system. The missing information required from such models is found in the velocity probability distribution for the real system. Statistical information of such detail is not likely to be available, but in many situations a specification of the mean velocity and spatial correlations will characterize uncertainty, provided the random velocity variations are sufficiently small. 
In summary, the actual water flow in a ground-water system while in a steady state condition is not random. A unique hydraulic gradient and hydraulic conductivity or transmissivity exist at each location, and constituent fluxes are all in a balance that results in a distinct flow pattern with perfectly well defined water flux at the boundaries. Uncertainty, however, is introduced by the inexact and incomplete measurement of hydraulic properties. Indeed, considerable spatial variability of hydraul ic conductivity, hydraulic potential, porosity, and storativity is common in an aquifer. Thus, any field sampling scheme, which is inherently incomplete due to practical restrictions, will reflect that spatial variability and give rise to stochastically determined hydraulic properties. Therefore, from an experimentalist's viewpoint, an aquifer consists of a random porous medium. Velocity is uncertain as a consequence of the combined random nature of hydraulic conducitivity $(K)$, hydraulic potential gradient $(\nabla H)$, and porosity $(\phi)$. The statistical variations of those transport parameters are related by Darcy's Law:

$$
\vec{v}=\frac{-K}{\phi} \nabla H .
$$

Variation in hydraulic conductivity is found to be mainly responsible for variaton in Darcy velocity (Eq. 5). The stochastic approach to dispersion in transport, therefore, has a major requirement that the random spatial variability in hydraulic conductivity be described in terms of its statistical parameters.

In a statistically homogeneous aquifer (second order stationary), the Kriging method provides the necessary estimates of means and covariances of the hydraulic properties (Delhomne 1979). That methodology, however, is not discussed here.

The Ensemble Concept

The stochastic approach to transport is based on the concept that a local. description of solute transport by Equations 1 and 5, for a particular set of measured hydraulic properties, constitutes only one realization or estimate of 
the actual transport. The fundamental principle is then that the actual transport description is represented by the expectation (or average) over a 11 possible realizations allowed by the relevant initial and boundary conditions. In this sense, initial and boundary conditions may also be uncertain. A collection or set of admissible realizations of transport is called an ensemble. For a deterministic description of initial or boundary conditions, the ensemble can be identified with the collection of possible flow velocity fields. Thus, the objective of a stochastic approach is to obtain the average of concentration distributions satisfying Eq. 1 for all possible velocity fields allowed by conditions on the transport problem. The concept of an ensemble of systems used here is similar to that introduced into statistical mechanics by Gibbs (1902), and into flow in porous media by Scheidegger (1954). Whereas their application is for the microscopic scale, the use here, however, is for the macroscopic scale. Predictions for a system are then obtained at the megascopic scale.

All stochastic transport approaches depend on a velocity ensemble, either implicitly or explicitly. In the cited references, all velocity ensembles are expressed implicitly by specifying the means and covariances. This report considers the explicit respresentation of an ensemble. In principle, expectations are taken over the entire theoretical ensemble, but in practice only an approximate and incomplete velocity ensembly may be available. Moreover, the individual members of a velocity ensemble may not always be separately observable or measurable in the actual ground water systems. In the layered aquifer example previously discussed, the ensemble members are observed as the velocities associated with each layer, assuming there is no velocity variation within each layer. A transport model, based on independent flow tube pathways, is another example with an observable ensemble. Now the layered aquifer were merged into a single system without altering the distribution of solute mass over velocities, an ensemble without directly observable members is produced. Because all solute molecules are identical, only a mass averaged velocity would be directly measurable or observable as the water velocity. Tracer experiments would be needed for each location in space to determine the fraction of mass crossing a test volume with a 
particular velocity. Thus, an ensemble yielding a particular mean and variance of the concentration may not be uniquely determined unless its members are actually observable.

Objectives and Limitations

This report is concerned with representation of dispersive mass transport by a stochastic convective flow. The classical convection equation (Eq. 1), with dispersion coefficient equal zero, is taken as the fundamental statement of solute conservation, and each concentration distribution is determined as a random function of stochastic velocity. The local Fickian dispersion term is neglected because it is usually small compared with dispersive effects of macroscopic velocity variation. In any case, however, local hydrodynamic dispersion can be represented by a velocity variation that can be superimposed on the macroscopic variation to yield a complete velocity distribution. Statistical behavior of the velocity field is assumed known, and the problem of relating it to variation in the hydraulic properties is not considered here. To simplify discussion, the mathematical exposition is done mainly in one dimension. This is done also because research into higher dimensional cases is still incomplete. The details of dispersion effects caused by chemical interactions with the porous medium are not discussed either. However, when sorbtion on the porous medium can be assumed in instantaneous equilibirum and represented by a retardation factor, the transport can be described in terms of a retarded stochastic velocity (velocity divided by retardation factor). The stochastic formulation, in terms of a velocity ensemble, is introduced and the connection between a velocity probability distribution and travel time is discussed. Conditions for the existence of an effective dispersion coefficient are derived in ensemble context. Two examples are given which show that an ensemble velocity probability density depends on the boundary conditions if the expected dispersion is Fickian.

An ensemble approach provides the basis for study of fundamental concepts, but is not generally useful as a computational tool because the complete velocity probability distribution is seldom known or even obtainable. On the other hand, a perturbation approach provides an efficient numerical method for estimating average and standard deviation of 
concentration in the case that random velocity variation is small relative to mean velocity. Oster and Gibbs (1979) applied the Keller perturbation method to a finite difference model of stochastic convective transport. 13 The particular form of the averaged transport equation, however, was not clarified. Therefore, an explicit equation that displays the dependence on a velocity covariance function is derived in the one dimensional case.

Random motion models for conceptualized solute particle movement, under arbitrary velocity variation conditions provide a particularly useful approach. An ensemble of hypothetical, but representative, solute particles can be moved simultaneously according to both a random walk describing local hydrodynamic dispersion and a megascopic velocity variation (see, for example, Smith and Schwartz, 1980). Such a method was applied by Matheron to discuss existence of an effective dispersion coefficient for a stratified flow system (Simpson 1979). Those results are reviewed since non-Fickian transport is demonstrated. Now most random motion models of transport utilize a Brownian random walk, which is more characteristic of diffusion rather than dispersion. Diffusion processes allow particles to move in all directions, whereas dispersion should not allow particles to move counter to the average local flow direction. Hence, a random motion model based on forward direction displacements is suggested. Developement of such models will be the subject of another report. It is mentioned here without providing details, however, that random motion models provide methods for direct construction of velocity ensemb les. 


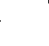




\section{DISPERSION AND STOCHASTIC CONVECTIVE TRANSPORT}

A. ENSEMBLE METHODS

Conservation Equations

The objective of the ensemble approach is to obtain the expected solute concentration by directly averaging over an ensemble of possible concentration distributions $c$ depending on position $\vec{x}$, time $t$, and a specific velocity field, $\vec{v}$. Each member of an ensemble of concentrations is required to satisfy conservation of solute mass as expressed by the continuity equaiton:

$$
\frac{\partial}{\partial t}(\phi c)+\nabla \cdot(\phi c \vec{v})=S(\vec{x}, t),
$$

where $S(\vec{x}, t)$ denotes a source on sink term In a saturated flow system, $\phi$ will denote porosity of the medium, whereas in an insaturated system it will alternatively represent the water content. Eq. 1, which describes concentration with respect to solution volume, when expressed in terms of concentration in the porous medium, $\rho=\phi C$, is:

$$
\frac{\partial \rho}{\partial t}+\nabla \cdot(\rho \vec{v})=S(\vec{x}, t)
$$

Conservation of fluid in the porous medium is described by the continuity equation:

$$
\frac{\partial \phi}{\partial t}+\nabla \cdot \vec{Q}=0
$$

where $\vec{Q}$ is the Darcy flux equal to $\phi \vec{v}$. Combining Equations 1 and 3 gives the convective transport equation:

$$
\frac{\partial C}{\partial t}+\vec{V} \cdot \nabla c=\frac{S(\vec{x}, t)}{\sigma} .
$$


For divergenceless flow, velocity satisfies $\nabla \cdot \vec{v}=0$, and water content also satisfies a convection equation:

$$
\frac{\partial \phi}{\partial t}+\vec{V} \cdot \nabla \emptyset=0
$$

Notice that under this condition, a one-dimensional system has a constant velocity; and without the presesents of sources or sinks, $C$ and $\phi$ would satisfy the same stochastic convection equation. If on the other hand, the flow is in incompressible saturated medium,

$$
\partial \phi / \partial t \equiv 0 ;
$$

and the Darcy flux in 1-D, Q, is constant. In this latter case, spatial variation of velocity is linked to that of porosity by

$$
V=Q / \phi
$$

Although constant over space, Q can take different values over an ensemble of flow systems. If the solute is a radioactive contaminant with decay constant $\lambda$ and sorbtion on the porous medium is described by a retardation factor $k$, then the sink term is:

$$
S=-\phi(K-1) \frac{\partial C}{\partial t}-\lambda K \phi c
$$

for solute concentration in units of activity (Curies).

The sink term in Equations 2 and 4 will be assumed equal to zero in the discussion of ensemble averages. Notice that Eq. 4 with the sink term Eq. 6 can be transformed into an equation of the same form by defining a retarded velocity $\vec{V} / K$ and considering the solutions of the form $e^{-\lambda t} c$.

The solute transport Equations 2 and 4 will in general define two different representations of concentration ensembles. Equation 2 is inherently a statement of solute mass conservation, whereas Eq. 4 is not without the 
constraint of Eq. 3. It is emphasized without details at this juncture in the discussion that a stochastic view of Eqs. 2 and 4 can lead to different physical interpretations depending on restrictions placed on velocity and $\phi$, contrary to a single deterministic view point. This difference in eqs. 2 and 4 is seen by defining the convective flux which is:

$$
\vec{U}=\rho \vec{V}=\overrightarrow{C Q}
$$

Notice that when $\phi$ is constant, Eq. 1 has the form of Eq. 2, and velocity satisfies the restriction of divergenceless flow.

Ensemble Expectation

An ensemble of concentration distributions is a collection of solutions or integrals of one of the convective transport equations. The ensemble is restricted to one of the representations Eqs. 2 or 4 . The collection of integrals is determined by the variety of velocity fields $\vec{v}$ and by the initial and boundary conditions included in the ensemble as random transport parameters. Thus in general the initial and boundary conditions may be uncertain as well as velocity, and velocity may be constrained by the fluid continuity Eq. 3. An ensemble expectation or average of concentration at each location and time is denoted by $\langle c\rangle$ or $\langle\rho\rangle$, and the expectation of any random variable associated with the ensemble is denoted by the symbol $<\cdot>$.

Only ensembles associated with flow velocity variations are considered here, and each ensemble can then be identified with a collection (set) of velocity fields $\vec{v}$ which is denoted as $\dot{E}$. The expectation determines a linear operation on functionals of the velocity, such as concentration integrals $c(\vec{x}, t ; \vec{v})$ depending on $\vec{v}$. It is required that a velocity probability distribution, denoted $P[\vec{v} ; \vec{x}, t]$, be defined on $\mathcal{E}$, which represents the probability of occurrence of a particular velocity field in the ensemble. Formally, expectations are expressed as integrations with respect to the ensemble probability distribution:

$$
\langle\vec{V}\rangle(\vec{x}, t)=\int_{\varepsilon} \vec{V} d P[\vec{v} ; \vec{x}, t]
$$


and

$$
\langle c\rangle(\vec{x}, t)=\int_{e^{0}} c(\vec{x}, t ; \vec{v}) d P[\vec{v} ; \vec{x}, t]
$$

for example, where $c(\vec{x}, t ; \vec{v})$ is a solution of Equations 1 or 4 . The expression Eq. 9 will be used also to represent the expectation of any functional of a random velocity. In some cases, $P[\vec{V} ; x, t]$ may represent a probability measure on $E$, and implied by that is the existence of a certain set of measurable subsets of $\varepsilon$ for which a probability is defined. That is, if $d$ contained in $E$ is measurable, then

$$
0 \leq P[\mathscr{d} ; \vec{x}, t] \leq P[\varepsilon ; \vec{x}, t]=1
$$

where

$$
P[d ; \vec{x}, t]=\int_{\mathscr{L}} d P[\vec{v} ; \vec{x}, t] .
$$

And if $\left\{\mathbb{S}_{i}\right\}$ is a (denumberable) collection of disjoint subsets with union equal $E^{\infty}$, then

$$
\Sigma_{i} P\left[d_{i} ; \vec{x}, t\right]=1
$$

For example, if $\varepsilon^{\infty}$ is a denumerable collection of velocities $\vec{v}_{j}$ representing distinct streamlines of some flow system, and the probability of each velocity is $p_{j}(\vec{x}, t)$ depending on location and time such that

$$
\sum_{i} p_{j}(\vec{x}, t)=1,
$$

then the measure is defined by

$$
P\left[\vec{v}_{j} ; \vec{x}, t\right]=p_{j}(x, t) \text {. }
$$

Expected concentration would be given by

$$
\langle c\rangle(\vec{x}, t) \equiv \Sigma_{i} p_{i}(\vec{x}, t) c\left(\vec{x}, t ; \vec{v}_{j}\right) \text {. }
$$


This expectation requires that the concentration solution exist for each velocity in $\varepsilon$, which in general corresponds to requiring proper measurability of $c(\vec{x}, t ; \vec{v})$. Moreover, this expectation may be infintte everywere when the ensemble is infinite, unless properties of the probability measure and concentration assure integrability.

In general, however, the ensemble expectation is not required to be representable as an integration with respect to a properly defined probability measure. Some examples of ensemble expectation will be given to clarify this concept. But first, since the ensemble method depends on performing an explicit averaging of integrals of the convective transport equation, some elementary solutions are described.

Solutions to Convective Transport Equation

To simplify the discussion, solutions are restricted to satisfy entirely either an initial value or boundary value formulation. An initial value problem will not include solute flux inputs or outputs, and the total solute mass is conserved in the system. On the other hand, a boundary value problem with either concentration or flux specified at the boundary will not include an inital distribution. Principles of superposition can be applied to yield the complete solution in general cases.

Initial Value Problems: One dimensional cases. The initial condition is

$$
c(x, 0)=c_{0}(x)
$$

where the initial distribution $C_{0}$ vanishes outside of a bounded region.

Position independent velocity: Assume the velocity is $V(t)$. The solution of Eq. 4 is:

$$
c(x, t)=c_{0}\left[x-\int_{0}^{t} v(Y) d \gamma\right]
$$

The solution of Eq. 2 yields the convective flux: 


$$
U(x, t)=V(t) \rho_{0}\left[x-\int_{0}^{t} V(r) d y\right] .
$$

where now po denotes the intial concentration.

Steady State Velocity: The case velocity depends only on position is perhaps the most relevant to a groundwater system. Solution of Eq. 4 is:

$$
c(x, t)=f[T(x)-t]
$$

where the travel time is

$$
T(x)=\int_{x_{0}}^{x} d y / V(y)
$$

and the function $f$ satisfies

$$
f[T(x)]=C_{0}(x)
$$

If the inverse $T^{-1}$ of Eq. 14 exists, which is the case for positive velocity, then the solution is:

$$
c(x, t)=c_{0}\left[T^{-1}(T(x)-t)\right],
$$

where $C_{0}(x)=0$ if $x \leq x_{0}$. The domain of definition is $0 \leq t \leq T(x)$. Alternatively, Eq. 2 can be used to determine the flux, eq. 7. Multiplying Eq. 2 by velocity $V(x)$ gives

$$
\frac{\partial U}{\partial t}+V(x) \frac{\partial U}{\partial x}=0 .
$$

The solution of Eq. 16 is:

$$
U(x, t)=f_{0}\left[T^{-1}(T(x)-t)\right]
$$


where

$$
f_{0}(x)=V(x) \rho_{0}(x)
$$

is the initial flux.

Notice that the water content Eq. 3 is solved in a similar way.

Boundary Value Problems: One dimensional cases. The boundary condition at $x=0$ is:

$$
c(0, t)=f(t)
$$

with $f(t)=0$, if $t \leq 0$.

Alternatively the flux is specified instead:

$$
U(0, t)=J(t)
$$

with $J(t)=0$, if $t \leq 0$.

Steady State Velocity: The solution of Eq. 4 is:

$$
C(x, t)=f[t-T(x)]
$$

with $x_{0}=0$ defining the travel time by Eq. 14. The corresponding solution of Eq. 2 is:

$$
\rho(x, t)=\frac{v(0)}{v(x)} f[t-T(x)]
$$

for $\rho(0, t)=f(t)$ instead. If the flux is specified by Eq. 19, the solution of Eq. 16 is:

$$
U(x, t)=J[t-T(x)]
$$


Higher Dimensional Solutions:

Position Independent Velocity: Assume that the velocity field depends only on time and initial conditions are:

$$
c(\vec{x}, 0)=c_{0}(\vec{x})=c_{0}\left(x_{1}, x_{2}, x_{3}\right)
$$

Then the solution of Eq. 4 is:

$$
c(\vec{x}, t)=c_{0}\left[\dot{x}-\int_{0}^{t} \vec{v}(\gamma) d \gamma\right]
$$

Travel Time Solution: Let the concentration be specified at the point $x_{0}$ :

$$
c\left(\vec{x}_{0}, t\right)=f(t)
$$

Consider solutions of the form:

$$
c(\vec{x}, t)=f[t-T(\vec{x})]
$$

Then $T(\vec{x})$ must satisfy

$$
\vec{V} \cdot \nabla T(\vec{x})=1
$$

Suppose now that $\vec{v}$ satisfies the curl condition:

$$
\nabla X\left(\frac{\vec{V}}{1 \vec{v} 1^{2}}\right)=0 .
$$

Then

$$
T(\vec{x})=T\left(\vec{x}_{0}\right)+\int_{\vec{x}_{0}}^{\vec{x}} \frac{\vec{v}}{1 \vec{v} 1^{2}} \cdot d \vec{x}
$$


defines a potential function with the integral being independent of path. That is, the field $\vec{V} /\left.1 \vec{V}\right|^{2}$ is conservative. Thus

$$
\nabla T(\vec{x})=\frac{\vec{v}}{1 \vec{v} 1^{2}}
$$

satisfies Eq. 25, and Eq. 24 with Eq. 26 is a solution of Eq. 4. Letting $T\left(x_{0}\right)=0$ makes the solutions take the specified value. Now

$$
f\left[t-T\left(\vec{x} ; \vec{x}_{0}\right) ; \vec{x}_{0}\right] \Delta \vec{x}_{0}
$$

with $f$ depending explicitly on $x_{0}$ is a solution also. Let $S$ be a domain of points $\vec{x}_{0}$ with finite volume. Then

$$
c(\vec{x}, t)=\int f\left[t-T\left(\vec{x}_{S} x_{0}\right) ; \vec{x}_{0}\right] d \vec{x}_{0}
$$

by superposition, is a solution if the integeral exists. In particular, a constant velocity field yields solutions Eq. 27.

A Stratified System: Consider a flow system with layers parallel the $x$-axis, and $\vec{V}=(V(x), 0)$ indicating no flow between the layers. Eq. 2 reduces to Eq. 16 for the $x$-component of flux. Then

$$
U(x, y, t)=J[t-T(x, y) ; y]
$$

is a solution for the flux with

$$
T(x, y)=\int_{x_{0}(y)}^{x} d z / V(z),
$$

where $x_{0}(y)$ is a curve for $0 \leq y \leq b$. Along the curve $x_{0}(y)$ the flux is specified as a boundary value: 


$$
U\left[x_{0}(y), y, t\right]=J(t ; y)
$$

Note that Eq. 29 is the travel time between the curve and point $(x, y)$ on a layer.

\section{B. ENSEMBLE EXAMPLES}

Some examples of velocity ensembles and expectations are now presented. It will be seen that even apparently simple convective transport systems manefest a vast variety of transport descriptions when the velocity is stochastic at each location and time. Initial and boundary conditions are deterministic in the calculation of expectations.

The most simple ensemble is for a one-dimensional system with constant velocity. Although simple in mathematical detail, it is far from being a trivial case, in as much as most of the physical behavior expected in two and three dimensional systems appears.

\section{Constant Velocity Ensembles}

The ensemble is characterized by a velocity probability density function (p.d.f.) which depends on location $x$ and time $t: p(v ; x, t)$. Its properties are $p(v ; x, t) \geq 0$ and

$$
\int_{-\infty}^{\infty} p(V ; x, t) d V=1
$$

Expectations are calculated as follows. A bar over a stochastic quantity will denote an expectation that is observable.

Initial Value Formulation: The average concentration is defined as

$$
\bar{c}(x, t)=\langle c\rangle \equiv \int c(x, t ; v) p(v ; x, t) d v
$$

where $c(x, t ; v)$ is a solution of the convection equation:

$$
\frac{\partial C}{\partial t}+v \frac{\partial C}{\partial x}=0
$$


For an initial value problem given by Equations 10 and 11 , the expectation is:

$$
\begin{aligned}
\bar{c}(x, t) & =\int_{-\infty}^{\infty} c_{0}(x-v t) p(v ; x, t) d v \\
& =\int_{-\infty}^{\infty} c_{0}(y) G(x, t ; y) d y
\end{aligned}
$$

where

$$
\begin{aligned}
G(x, t ; y)= & \int \delta(x-v t-y) p(v ; x, t) d v . \\
& =p[(x-y) / t ; x, t] / t
\end{aligned}
$$

The function of Eq. 33 represents a stochastic Green's function (Adomian 1970). It represents the kernel of an inverse integral operator for an as yet unknown resultant transport equation. An important problem is to determine under what conditions the expected concentration; Eq. 30, represents a solution of the classical convective-dispersion equation for some velocity and dispersion coefficient. An equivalent convective-dispersion system exsts for the ensemble if Eq. 30 satisfies

$$
\frac{\partial \bar{C}}{\partial x}+\frac{\partial}{\partial x}(u \bar{c})=\frac{\partial}{\partial x}\left(D \frac{\partial \bar{c}}{\partial x}\right)
$$

for some velocity $u(x, t)$, which may not necesarily equal the expected velocity, and non-negative dispersion coefficient $D(x, t)$. (Water content $\phi$ is assumed constant.) If the velocity u equals the ensemble expected velocity,

$$
\bar{V}(x, t)=\int_{-\infty}^{\infty} V p(V ; x, t) d V,
$$

then $D$ is an effective dispersion coefficient. These conditions establish precisely when the stochastic convective transport describes dispersion 
according to Fick's law. However, an equivalent convective-dispersive system as represented by a proper pair of functions $u$ and $D$ will not necessarily be uniquely determined; and moreover, the particular pair satisfying Eq. 34 will in general depend on the initial concentration distribution as well as on the stochastic Green's function. For example, suppose that a particular concentration eq. 32 satisfies eq. 34 , and let $u^{\star}$ and $D^{\star}$ be selected such that

$$
u^{\star} \bar{C}-D^{\star} \frac{\partial \bar{C}}{\partial x}=0
$$

Then $\bar{c}$ also satisfies the convective dispersion equation for the pair of functions $\bar{u}=u^{+} u^{\star}$ and $\bar{D}=D+D^{\star}$. It is required that $\bar{D}$ is non-negative and $\bar{u}$ flows in the correct direction so that this pair of functions constitutes a physically realistic system. The corresponding two equivalent convective-dispersive systems would in general determine two distinct modes for propagation of an initial concentration distribution different than the original $C_{0}$. The resultant non-uniqueness appears unavoidable unless the velocity u can be uniquely established, possibly as being the ensemble mean eq. 35. Now in view of the expectation's representation (Eq. 32), in order that it satisfy Eq. 34 for all initial concentration distributions $C_{0}, E q .33$ must satisfy Eq. 34. Only when $D(x, t)$ can be determined independently of initial values does it constitute a truely effective dispersion coefficient representing inherent stochastic flow properties of a system. That stronger condition, however, will not be required in the definition. An effective dispersion coefficient is allowed to change with different initial or boundary values. Thus when the stochastic Green's function Eq. 33 is a solution of some convective-dispersion Eq. 34 and $u$ is identical to the expected velocity Eq. 35, then an effective dispersion coefficient, which is independent of initial concentration, exists for the ensemble. For the expectation Eq. 32 to satisfy the same inital condition, Eq. 33 must be the proper Green's function for $\mathrm{Eq} .34$.

Two important examples of constant velocity ensembles are now considered. Observe that if the velocity p.d.f. is independent of position then 


$$
p^{\star}(x ; t)=p(x / t ; t) / t
$$

determines a probability density function for $x$. In that case, the stochastic Greens function Eq. 33 used in Eq. 32 is given as $p^{\star}(x-y ; t)$ in terms of Eq. 36. The definition Eq. 36 provides a direct link between the stochastic parameters of the velocity ensemble and the $u$ and $D$ of possible equivalent convective-dispersive systems. Indeed, such a system exists if Eq. 36 itse if is a solution of Eq. 34. (Refar to Appendix A for details.) Now suppose that the velocity p.d.f. is Gaussian:

$$
p(v ; t)=\frac{1}{\sqrt{2 \pi} \mu} e^{-(V-\bar{V})^{2} / 2 \mu^{2}}
$$




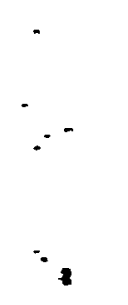


Suppose that the mean $\bar{v}$ and varlance $\mu^{2}$ are independent of time so that Eq. 37 is a stationary Gaussian distribution. Then Eq. 36 is given by

$$
p *(x ; t)=\frac{1}{\sqrt{2 \pi} \sigma} e^{-(x-\bar{V} t) 2 / 2 \sigma^{2}}
$$

with $\sigma=\mu t$. And the effective dispersion coefficient is

$$
D=\mu^{2} t
$$

Thus the effective dispersion increases linearly with time for a stationary Gaussian velocity ensemble. Now suppose instead that Eq. 38 is the classical solution of the convective - diffusion equation for constant velocity $\bar{V}$ and dispersion coefficient $D$. Then $\sigma^{2}=2 D t$, and the variance in Eq. 37 is:

$$
\mu^{2}=2 D / t
$$

Thus the variance of a Gaussian velocity ensemble decreases inversely with time if the effective dispersion coefficient is constant (see Appendix $A$ for proof details).

The previous examples should not be interpreted to mean that most transport systems have effective dispersion coefficients. In fact, such systems are not common for a megascopic scale.

Boundary Value Formulation: It is assumed in this discussion that the velocity p.d.f is defined for $V \geq 0$ so that solute released at the origin moves only into the region $x \geq 0$. However, this restriction is not required in general. To simplify the discussion, water content is taken constant and equal to unity so that $c$ equals $p$, and the stochastic convective flux is:

$$
U(x, t ; v)=V C(x, t ; v)
$$


The two boundary condition cases are:

$$
\begin{aligned}
& \text { (i) } c(0, t ; v)=f(t) \\
& \text { (ii) } U(0, t ; v)=f(t) \text {. }
\end{aligned}
$$

Case (i) is for a deterministic concentration, and case (ii) is for a deterministric flux. In each case the other quantity associated according to Eq. 41 is stochastic. Observe that concentration and flux satisfy the same convection Equations 16 and 31 . The respective solutions are:

$$
\begin{aligned}
& \text { (i) } c(x, t ; v)=f(t-x / v) \\
& \text { (ii) } c(x, t ; v)=f(t-x / v) / v \text {. }
\end{aligned}
$$

The expectations, defined by $\mathrm{Eq} .30$, are given by

$$
\bar{c}(x, t)=\int_{0}^{\infty} f(\gamma) G(x, t ; \gamma) d \gamma
$$

where

$$
\text { (i) } \begin{aligned}
G(x, t ; \gamma) & =\left\langle\delta_{+}(\gamma-t+x / v)\right\rangle \\
& =x p[x /(t-\gamma) ; x, t] /(t-\gamma)^{2} \\
\text { (ii) } G(x, t ; \gamma) & =\left\langle\delta_{+}(\gamma-t+x / V) / V\right\rangle \\
& =p[x /(t-\gamma) ; x, t] /(t-\gamma) .
\end{aligned}
$$

$\delta_{+}(x)$ denotes the delta distribution for $x \geq 0$. It is required in Eq. 44 that $f(t)=0$ if $t<0$ and $p(v ; x, t)=0$ if $v<0$, so that the upper limit of integration is replaceable by $t$.

Again the stochastic Green's function Eq. 45 must be a solution to some convective-dispersion Eq. 34 in order that an equivalent convective-dispersion 
system, which is independent of boundary values $f$, exists for the particular velocity ensemble. Boundary conditions satisfied by a Green's function, however, can be different from those imposed on the original stochastic convective flow.

Because the convective-dispersion equation is known to provide a correct description of transport at the macroscopic scale, in systems such as soil columns, it is important to determine when such Fickian transport can be represented by a constant velocity ensemble. This prolem will be examined for Eq. 34 depending on a constant velocity and dispersion coefficient. A velocity ensemble expectation can be transformed into a travel time expectation by introducing into Eq. 30 the substitution $\gamma=x / V$ and by defining

$$
p^{\star}(r ; x, t)=x p(x / r ; x, t) / \gamma^{2}
$$

The function Eq. 46 defines a travel time p.d.f. for $r \geq 0$ if and only if $p(V ; x, t)$ is a constant velocity p.d.f. Stochastic Green's function and travel time p.d.f. are then related as follows:

$$
\begin{aligned}
& \text { (i) } G(x, t ; \gamma)=p^{\star}(t-\gamma ; x, t) \\
& \text { (ii) } G(x, t ; \gamma)=(t-\gamma) p^{\star}(t-\gamma ; x, t) / x .
\end{aligned}
$$

Then proof that a particular solution of the convective-dispersion Eq. 34 determines an equivalent constant velocity ensemble requires demonstration that it is expressed by Eq. 44 and that the Green's function defines a travel time p.d.f. by Eq. 47. It is well known that Green's functions for Eq. 34 are constructed from the fundamental Gaussian solution: 15

$$
g(x, t)=\frac{1}{\sqrt{2 \pi D t}} e^{-(x-u t)^{2} / 4 D t}
$$


An interesting property of Eq. 48 is that it defines a travel time p.d.f. by

$$
p^{*}(\gamma ; x)=u g(x, \gamma)
$$

and

$$
\bar{c}(x, t)=\int_{0}^{t} f(\gamma) p^{\star}(t-\gamma ; x) d \gamma
$$

is a solution of Eq. 34 satisfying initial condition

$$
\bar{c}(x, 0)=0
$$

for the travel time p.d.f. Eq. 49. This solution is defined for $x>0$ and the boundary value of concentration is given as an average of $f$ :

$$
\bar{c}(0, t)=\int_{0}^{\infty} f(t-\gamma) p^{\star}(\gamma ; 0) d \gamma(t>0) .
$$

Hence, Eq. 49 determines an equivalent ensemble, but the boundary conditions satisfied by the stochastic convective system for Eq. 42 (i) are not necessarily the same as those of the expectation Eq. 50. The velocity p.d.f. corresponding to Eq. 49 is:

$$
p(v ; x)=\frac{u}{\sqrt{\pi a}}{\frac{e}{v^{3 / 2}}}^{-(v-u)^{2} / a v}
$$

with $\mathrm{a}=40 / \mathrm{x}$. And the expected velocity $\mathrm{V}$ for Eq. 53 equals $u$. Note that

$$
\frac{2 e^{2 \beta}}{\sqrt{\pi}} \int_{0}^{\infty} e^{-x^{2}-\beta^{2} / x^{2}} d x=1
$$


is the fundamental integral used to prove that varlous functions derived from Eq. 48 by transformation are indeed probability densities. This example demonstrates that $D$ is an effective dispersion coefficient for the ensemble described by Eq. 53 .

Duhame 1 's theorem or Laplace transform methods can be applied to derive the solution of Eq. 34 subject to initial condition Eq. 51 and boundary condition Eq. 42 (i). The Green's function for Eq. 44 is:

$$
\begin{aligned}
G(x, t ; \gamma) & =\frac{x}{2 \sqrt{\pi D}} \frac{e^{-(x-u(t-r))^{2} / 4 D(t-r)}}{(t-\gamma)^{3 / 2}} \\
& =x g(x, t-\gamma) /(t-\gamma) .
\end{aligned}
$$

(See appendix $B$ for details.) Since the integral of Eq. 55 with respect to $r$ is singular (infinite) at $x=0$, spectal evaluation of an indeterminant form shows that $G(x, t ; r) \rightarrow \delta_{+}(r-t)$ as $x \rightarrow 0$ and Eq. 44 for Eq. 55 satisfies the required conditions. The travel time p.d.f. corresponding to Eq. 55 satisfies the required conditions. The travel time p.d.f. corresponding to Eq. 55 is:

$$
p^{\star}(r ; x)=\frac{x}{r} g(x, r),
$$

and the associated velocity p.d.f. Is:

$$
p(v ; x)=\frac{1}{\sqrt{\pi a}} \frac{e^{-(V-u)^{2} / a v}}{\sqrt{V}}
$$

with $a=4 D / x$. Expected velocity for Eq. 2.57 is:

$$
\nabla=u+2 D / x
$$

Therefore Eq. 57 represents an ensemble having an equivalent convectivedispersive system, but the expected velocity Eq. 57 is greater than $u$. This 
shows that $D$ is not a true effective dispersion coefficient. An effective dispersion coefficient $\bar{D}(x, t)$, however, can be established by equating the total Fickian flux given by

$$
\bar{F}(x, t)=u \bar{C}-D \frac{\partial \bar{C}}{\partial x}
$$

to that determined by $\vec{V}$ and $\bar{D}$ :

$$
\bar{F}(x, t)=\nabla \bar{C}-\bar{D} \frac{\partial \bar{C}}{\partial x}
$$

Then

$$
\bar{D}(x, t)=D+(2 D / x) \bar{C} / \frac{\partial \bar{C}}{\partial x}
$$

defines an effective dispersion coefficient for $x$ and $t$ such that $E q .60$ is non-negative. The definition Eq. 60, will clearly depend on the specific boundary values of concentration. This example demonstrates that an effective dispersion coefficient is not likely to represent a fundamental property of the medium, because it depends on initial and boundary conditions.

A special case for $u \equiv 0$ provides an example of particular interest, since then the Fickian flux Eq. 58 can flow in either direction, depending on the boundary concentration. But the expected convective $f l u x,\langle U(x, t ; v)\rangle$, flows only in the expected velocity's direction. This demonstrates that the expected convective flux may not be conservative, in as much as the Fickian flux Eq. 58 constitutes the observable conservative flux for this example.

Next consider Eq. 42 (ii), the case when flux is specified at the boundary. Such a case is perhaps most relevant to transport problems involving an assumed solute release rate into a groundwater system. Again the objective is to determine solutions of the convective-dispersion equation 
which have equivalent ensemble representations. The flux boundary conditions for the deterministic Fickian transport are divided into the following two cases:

$$
\begin{aligned}
\left(u \bar{c}-D \frac{\partial \bar{c}}{\partial x}\right)_{x=0} & =u f(t) \\
& =f(t)
\end{aligned}
$$

The transport domain is infinite, $x \geq 0$, and $c\left(x=+_{\infty}, t\right)=0$ for all $t$ is assumed. A special transformation property of Eq. 34 can be applied to utilize the previous results. For $u$ and $D$ independent of $t$, the flux (Eq. 58) also satisfies the convective-dispersion equation:

$$
\frac{\partial \bar{F}}{\partial t}+u \frac{\partial \bar{F}}{\partial x}=D \frac{\partial^{2} \bar{F}}{\partial x^{2}}
$$

The boundary conditions Eq. 61 for flux are similar to those for concentration specified at $x=0,{ }^{\circ}$ and, therefore

$$
\bar{F}(s, t)=\int_{0}^{t} f(t-\gamma) \frac{x}{\gamma} g(x, \gamma) d \gamma
$$

for case (ii) of Eq. 61. Flux for case (i) is simply multiplied by $u$. Applying the definition Equations 58 to 44 gives that the Green's function for the solution satisfies:

$$
u G(x, t ; \gamma)-D \frac{\partial G}{\partial x}(x, t ; \gamma)=\frac{x}{t-\gamma} g(x, t-\gamma)
$$

Imposing the condition $G(x, t ; \gamma) \rightarrow 0$ as $x \rightarrow \infty$, the solution of Eq. 64 is found to be

$$
G(x, t ; \gamma)=\frac{e^{u x / D}}{D(t-\gamma)} \int_{x}^{\infty} e^{-u y / D} y g(y, t-\gamma) d y
$$


or

$$
=2 g(x, t-\gamma)-\frac{u}{D} \int_{0}^{\infty} e^{-u y / D} g(x+y, t-\gamma) d y .
$$

An equivalent ensemble exists for each case Eq. 61, if the Green's function Eq. 65 determines a travel time p.d.f. for the stochastic convective system with boundary conditions Eq. 42 .

The required travel time p.d.f. must be given by

$$
\begin{aligned}
& \text { (i) } p^{\star}(\gamma ; x)=2 p_{0}^{\star}(\gamma ; x)-\frac{u}{D} \int_{0}^{\infty} e^{-y u / 0} p_{0}^{\star}(\gamma ; x+y) d y \\
& \text { (ii) } p^{\star}(\gamma ; x)=2 p_{0}^{\star}(\gamma ; x)-\frac{u}{D} \int_{0}^{\infty} e^{-y u / 0} \frac{x}{x+y} p_{0}^{\star}(\gamma ; x+y) d y
\end{aligned}
$$

for eacin case, where

$$
\begin{aligned}
& \text { (i) } p_{0}^{\star}(\gamma ; x)=u g(x, \gamma) \\
& \text { (ii) } p_{0}^{\star}(\gamma ; x)=\frac{x}{\gamma} g(x, \gamma)
\end{aligned}
$$

Direct integration shows that Eq. 66 determines a p.d.f for (i) but Eq. 67 does not for ( $i i)$. However, case ( $i i)$ is represented by Eq. 66 as an expectation of another stochastic concentration, $f(t-x / v) u$. Thus it has been demonstrated that both flux and concentration for the boundary value problem Eq. 61 are represented as ensemble expectations. The ensembles for each quantity, however, are different. Indeed, flux is given by

$$
\bar{F}(x, t)=\int_{0}^{\infty} F(x, t ; \gamma) p_{0}^{\star}(\gamma ; x) d \gamma
$$


for each case Eq. 67 where the stochastic flux is given by

$$
\text { (i) } \begin{aligned}
F(x, t ; \gamma) & =\frac{x}{\gamma} f(t-\gamma) \\
\text { (ii) } F\left(x, t^{\prime} \gamma\right) & =f(t-\gamma)
\end{aligned}
$$

and satisfies

$$
U(x, t ; V)=F(x, t ; x / V)
$$

But the respective concentration ensembles are represented by Eq. 66 . Hence this example demonstrates that the observable concentration and flux may not be representable by the same ensemble. On the other hand, the expected convective flux derived from the ensemble for the observable expected concentration may not be a conservative flux. Specifically, $\langle U\rangle(x, t)$ for travel time p.d.f. Eq. 66 may not equal the conservative Fickian flux Eq. 69.

It can be shown, furthermore, that the observable conservative flux may not be representable by any fixed ensemble expectation of the stochastic convective flux Eq. 41. For example, returning to the previous case with concentration specified at $x=0$, calculation of flux Eq. 58 for Eq. 56 yields

$$
F(x, t)=\int_{0}^{\infty} F(x, t ; \gamma) p^{*}(\gamma ; x) d \gamma
$$

where the stochastic flux is:

$$
F(x, t ; y)=f(t-\gamma)\left(\frac{u+x / y}{2}-\frac{D}{x}\right)
$$

Now this flux does not determine a conservative, stochastic convective flux according to Eq. 71. Moreover, a travel time p.d.f. such that

$$
F(x, t)=\int_{0}^{\infty} f(t-\gamma) p_{0}^{\star}(\gamma ; x) d \gamma
$$


holds for all $f(t) \geq 0$ does not exist, in as much as Eq. 72 can be negative but the expected convective flux is non-negative. Notice that dependence on $D$ is the cause of negative flux. Apparently, the observed flux F can always be expressed as an expectation Eq. 72 for some stochastic flux $F(s, t ; \gamma)$.

Conservative Convective Flux: The foregoing ensemble examples indicate that observable values (denoted by a bar) and expectations of convective transport qualities are not necessarily identical. Observable values of the conjugate transpot quantities, concentration and flux, must satisfy the conservation equation:

$$
\frac{\partial \bar{C}}{\partial t}+\frac{\partial \bar{F}}{\partial x}=0
$$

Here the flux need not be Fickian as in Eq. 58. When the observed concentration equals its ensemble expectations Eq. 30 , then the conservative observed flux is given by

$$
\bar{F}(x, t)=\int F(x, t ; V) p(V ; x, t) d V
$$

for some possibly non-conservative stochastic flux $F(x, t ; V)$. Expected convective flux, however, equals the expectation of the conservative stochastic flux Eq. 41, and it need not satisfy Eq. 74. (Note that fluxes satisfying Eq. 74 can differ by an arbitrary additive function of t.) The expected convective flux, which is

$$
\langle U\rangle(x, t)=\int V c(x, t ; V) p(V ; x, t) d V,
$$

is conservative only if the stochastic flux $F$ equals Eq. 41 . On the other hand, if Eq. 76 constitutes an observed flux for a particular system, then the expected concentration may not be the conjugate observed value satisfying Eq. 74 . 
Conditions on the ensemble velocity p.d.f. such that Eq. 76 is conservative are established by substituting Eq. 76 into Eq. 74 for Eq. 75:

$$
\int c(x, t ; V) \quad\left[\frac{\partial p}{\partial t}+V \frac{\partial p}{\partial x}\right] d V=0
$$

Then an ensemble is conservative for all initial or boundary value formulations if the p.d.f. satisfies

$$
\frac{\partial p}{\partial t}(v ; x, t)+v \frac{\partial}{\partial x} p(v ; x, t)=0
$$

Therefore every conservative ensemble is of the form:

$$
p(v ; x, t)=p_{0}(x-v t ; v),
$$

for some function $p_{0}(x ; V)$.

A relationship between ensembles for the case each observed quantity is given by an expectation can be pursued in a similar way. Suppose $p(v ; x, t)$ and $p_{0}(v ; x, t)$ represent ensembles for stochastic concentration and flux, respectively. For an initial value formulation, position independence is assumed, and a travel distance p.d.f. is defined by Eq. 36. The observed expectations are:

$$
\begin{aligned}
& \bar{c}(x, t)=\int_{-\infty}^{\infty} f(x-y) p^{\star}(y ; t) d y \\
& \bar{F}(x, t)=\int_{-\infty}^{\infty} \frac{y}{t} f(x-y) p_{0}^{\star}(y ; t) d y .
\end{aligned}
$$


Therefore conjugate conservative ensembles such that Eq. 74 holds for all initial distributions satisfy:

$$
\frac{\partial p^{\star}}{\partial t}(x ; t)+\frac{\partial}{\partial x}\left(\frac{x}{t} p_{0}^{\star}(x ; t)\right)=0
$$

Assuming time independence for the boundary value formulations Eq. 42, the observed expectations are given by

$$
\bar{c}(x, t)=\int_{0}^{\infty} c(x, t ; \gamma) p^{\star}(\gamma ; x) d \gamma
$$

where

$$
\begin{aligned}
& \text { (i) } \quad c(x, t ; \gamma)=f(t-\gamma) \\
& \text { (ii) } \quad c(x, t ; \gamma)=\frac{\gamma}{x} f(t-\gamma),
\end{aligned}
$$

and by Eq. 69 with Eq. 70 for the flux. Conjugate conservative ensembles must satisfy the following in each case:

$$
\begin{aligned}
& \text { (i) } \frac{\partial p^{\star}}{\partial t}(t ; x)+\frac{\partial}{\partial x}\left(\frac{x}{t} p_{0}^{\star}(t ; x)\right)=0 \\
& \text { (ii) } \frac{\partial}{\partial t}\left(\frac{t}{x} p^{\star}(t ; x)\right)+\frac{\partial}{\partial x} p_{0}^{\star}(t ; x)=0
\end{aligned}
$$

where $p^{\star}(0 ; x)=0$ for $x>0$ is assumed.

In summary, a number of general inferences can be deduced from the study of constant velocity ensembles. It appears that the ensemble approach encompasses Fickian transport described by the convective-dispersion equation in terms of a dispersion coefficient. An unproven conjecture is that every Fickian system with spatially dependent velocity and dispersion coefficient has an equivalent ensemble for stochastic convective transport. This is easily verified for an initial value formulation as follows. Let $g(x, t)$ 
denote the fundamental solution of Eq. 34 for an initial delta distribution. As a conservation equation, Eq. 34 assures that $g(x, t)$ defines a p.d.f. for $x$. The solution for any initial solute distribution is:

$$
\bar{c}(x, t)=\int_{-\infty}^{\infty} f(y) g(x-y, t) d y
$$

A constant velocity ensemble is then defined by

$$
p(v ; t)=\operatorname{tg}(v t, t)
$$

A general proof, however, would require consideration of all initial and boundary value cases. Fickian transport systems having an equivalent ensemble characterize the stochastic transport described by an effective dispersion coefficient. It was shown that only stochastic systems yielding a Greens function solution to the convective-dispersion equation can possibly be represented by an effective dispersion coefficient. The difficulties with the concept of an effective dispersion coefficient are that it may not exist or may not be uniquely determined. Moreover, the examples show that an effective dispersion coefficient may depend on time and boundary conditions. Therefore such a coefficient is not an inherent property of the porous medium alone, but depends on external conditions--a result contrary to the concept of local macroscopic dispersion.

Two concepts of observability have been thus far introduced. In the first meaning, observable refers to the direct measurability of the individual ensemble members (velocity fields). An observable ensemble is composed of directly measurable realizations of transport, such as the concentration within each layer of a stratified aquifer, for example. The ensemble's velocity p.d.f. represents the probability of observing certain sets of members. In the second meaning, an observed value of the transport quantities, concentration and flux, designates an ensemble predicted value. An ensemble expectation of a measurable quantity represents an observed value. 
It was shown that ensemble expectations and observed values of transport quantities may not coincide. Observed values of concentration and flux satisfy the conservation equation, whereas the expected values may not. For example, if the average concentration over a layered aquifer is in observable, then the average flux may not be the associated observable conservative flux.

\section{Spatially Variable Ensembles}

Perhaps the most common ground water transport problem involves a steady state system with a spatially varying water velocity. In such cases uncertainty is only a consequence of spatial effects and is independent of time. To reflect this condition the velocity field ensemble is also independent of time, or stationary. In the mathematical theory of ground water flow, the velocity is usually visualized as a continuously changing quantity, deterministically established by Darcy's law (Eq. 5 in Chapter 1) at each location. However, in practice velocity is approximated at some observation scale as being piece-wise constant over a regional subdivision. The constant velocity values at each location are always imperfectly known, and therefore are stochastic.

A more general concept of an ensemble expectation is explained in this section. The methodology is not meant to provide a practicable computational approach, but instead is required in order to fully clarify the foundations of a stochastic viewpoint. The discussion is restricted to the deterministic boundary value cases Eq. 42. Since the integrals for concentration Eq. 20 and flux Eq. 22 are identical in form, it is sufficient to consider only concentration. Thus the objective is to give a more detailed definition of the symbol Eq. 9, for one dimensional systems.

Symbolically the expected concentration is denoted by

$$
\bar{c}(x, t)=\int_{\varepsilon} f\left[t-\int_{0}^{x} d y / V(y)\right] d P[V ; x]
$$

where $\mathcal{E}$ is the stationary velocity ensemble, and the integral represents some method of averaging over $\varepsilon$. Clearly, there can be many ways of defining the 
average over a particular ensemble, and only experimental verification can determine if a method is physically correct. Some possible ensemble will now be defined.

Example 1. Let $0=x_{0}<x_{1}<x_{2}<\ldots<x_{n}=L$ be a fixed partition of the interval $x=0$ to $x=L$. The ensemble $\mathcal{E}$ is all step functions defined over the partition: $V(x)=V_{i}$ (a constant) for $x_{i-1<x \leq x_{i}}$, $i=1, \ldots, n$. Velocity is not defined for $x>L$. A joint probability density function $p_{n}\left(v_{1}, \ldots, v_{n}\right)$ is assumed to exist, and expectation is

$$
\bar{c}(x, t) \equiv \int_{R^{m}} f\left(t-\sum_{i=1}^{m} \Delta x_{i} / v_{i}\right) p_{m}\left(v_{1}, \ldots, v_{m}\right) d v_{1} \ldots d v_{m}
$$

where $\Delta x_{i}=x_{i}-x_{i-1}$ for $i<m$ and $\Delta x_{m}=x-x_{m-1}$ when $x_{m-1}<x_{\leq} x_{m}$ for $m \leq n$. $\quad P_{m}\left(v_{1}, \ldots, v_{m}\right)$ for $m<n$ is the marginal p.d.f. given by

$$
p_{m}\left(v_{1}, \ldots, v_{m}\right)=\int_{R^{n-m}} p_{n}\left(v_{1}, \ldots, v_{n}\right) d v_{m+1} \ldots d v_{n} .
$$

$\mathrm{R}^{\mathrm{n}}$ denotes multi-dimensional integration over $\mathrm{n}$ dimensional space, $-\infty<V_{j<+\infty}$ for $i=1, \ldots, n$. The expectation is then defined by Eq. 85 only if the integral exists for the particular boundary value $f$. Note that the conditional expected velocity is:

$$
\left\langle V(x) / V^{\prime}(y)\right\rangle=\int_{R} V_{m} p_{m}\left(V_{1}, \ldots, v_{m-1}^{\prime}, v_{m}\right) d v_{m}
$$

for $V(y)=V^{\prime}(y)$ when $y \leq x_{m-1}$ where $x_{m-1}<x \leq x_{m}$, and the expected value is:

$$
\langle V(x)\rangle=\int_{R^{m}} v_{m} P_{m}\left(v_{1}, \ldots, v_{m}\right) d v_{1} \ldots d v_{m} .
$$

A special case of this ensemble is obtained by taking $p_{m}$ equal to the independent product of $n$ constant velocity probability densities, 
$p_{1}\left(V_{1}\right) p_{2}\left(V_{2}\right) \ldots p_{n}\left(V_{n}\right)$. For example, $p_{i}\left(V_{i}\right)$ could be the p.d.f. associated with a mean velocity and dispersion coefficient for the region (interval) $x^{i-1<x \leq x_{j}}$. A larger ensemble can be constructed by taking a union over a finite collection of ensembles defined for different intervals $(0, L)$ and partitions. The new expectation could equal the mean of expectations for the ensemble collection.

Example 2. A modification of the first example is constructed by allowing discontinuous integrals for the convection equation solutions. The construction, however, has the physical constraint that flux is continuous across the interface of adjacent intervals. Then solute outflow from an interval provides the inflow to the next interval as follows. For each $i=2, \ldots, n$ define $f_{i}(t)$ such that

$$
v_{i} f_{i}(t)=v_{i-1} f_{i-1}\left(t-x_{i-1} / v_{i-1}\right)
$$

$m=1, \ldots, n$, with $x_{0}=0$. Notice that $f_{m}$ depends on the preceeding velocities $v_{1}, \ldots, v_{m-1}$ for $m \geq 2$. The expected concentration is then given by

$$
\bar{c}(x, t)=\int_{R^{m}} c\left(x, t: v_{1}, \ldots, v_{m}\right) p_{m}\left(v_{1}, \ldots, v_{m}\right) d v_{1} \ldots d v_{m} .
$$

Apparently, Eq. 89 constructs the solution to Eq. 21. This example demonstrates that the expectation will depend on the particular construction of stochastic concentration distributions.

Example 3. The previous examples represent ensembles based on a finite dimensional space of velocities. An infinite dimensional example is provided by wiener measure in the space of continuous functions.(16) The measure is constructed from an infinite product of Gaussian probability distributions, and integration of continuous, bounded functionals of velocity can be defined. Since this example is beyond the mathematical scope of this report, details are not given, and the review article by Gel'Fand and Yaglon (1960) may be consulted. 
Example 4. A rigid streamline model is constructed as follows. The velocity ensemble $E$ is described by a set of velocity streamlines $V(x ; a)$, which depend on a parameter $a$. The parameters a are assumed to be members of a measure space $\Omega$ with a probabllity measure $\omega(\alpha)$ defined on 1 t. Velocities $V(x ; \alpha)$ must be integrable with respect to $w(a)$. A particular example is all $(n-1)$ degree polynomials in $x$ with coefficients $\alpha=\left(\alpha_{1}, a_{2}, \ldots, a_{n}\right)$. The expectation of velocity Eq. 8 is:

$$
\nabla(x)=\int_{\Omega} V(x ; \alpha) d w(\alpha),
$$

and that of concentration Eq. 84 is:

$$
\bar{c}(x, t)=\int f[t-T(x ; \alpha)] d \omega(\alpha)
$$

where

$$
T(x ; \alpha)=\int_{0}^{x} d y / V(y ; \alpha)
$$

is the travel time along the path a. The most useful ensemble representation is obtained by defining a corresponding travel time p.d.f. by

$$
p^{\star}(r ; x)=\int_{\Omega} \delta[\gamma-T(x ; \alpha)] d \omega(\alpha) .
$$

Then in terms of Eq. 11,

$$
\bar{c}(x, t)=\int_{0}^{\infty} f(t-\gamma) p^{\star}(r ; x) d \gamma
$$

where travel time is assumed non-negative. 
3. Purely Random, Stationary, Gaussian Processes Ensembles

The previous ensemble examples are based directly on probability density functions for velocity, and expectation is expressed explicitly as an integration. However, the transport equation satisfied by average concentration is not obtained. It is not known either if the resulting transport deviates from Fickian behavior. In this example, a very special ensemble characterized by mean and variance of velocity is discussed. An extreme case for which velocity is uncorrelated between different times or locations and its variance is constant is described by a purely random, stationary Gaussian process.

A function $w(x)$ satisfing such a process is characterized by the following conditions (Fox 1972):

$$
\begin{aligned}
& \langle W(x)\rangle=0 \\
& \langle W(x) W(y)\rangle=2 \lambda \delta(x-y),
\end{aligned}
$$

and: a) expectations of odd numbered products are zero:

$$
\left\langle w\left(x_{1}\right) w\left(x_{2}\right) \ldots w\left(x_{2 n-1}\right)\right\rangle=0 \quad(n=1,2, \ldots)
$$

b) even products satisfy:

$$
\begin{aligned}
\left\langle w\left(x_{1}\right) \ldots w\left(x_{2 n}\right)\right\rangle & =\frac{1}{2^{n} n !} \sum_{\rho} \prod_{j=1}^{n}\left\langle w\left(x_{\rho}(2 j)\right) w\left(x_{\rho}(2 j-1)\right)\right\rangle \\
& =\frac{1}{2^{n} n !} \sum_{\rho} 2^{n} \lambda^{n} \prod_{j=1}^{n} \delta\left(x_{\rho}(2 j)^{-x_{\rho}}(2 j-1)\right)
\end{aligned}
$$

where $\rho$ denotes sum over the set of permutations of order $(2 n) !$. Fox proved the following theorem for $w(x)$ satisfying Eq. $96(a)$ and (b): 


$$
\left\langle\exp \left[\operatorname{lig}_{0} \int^{x} w(y) d y\right]\right\rangle=e^{-\lambda a^{2} x}
$$

where $i=\sqrt{-1}$. Although the paper by Fox discusses another subject, Eq. 97 is general and applies to the ensemble study.

By using the property that the expectation operation and Fourier transform with respect to time commute, Eq. 97 can be applied to compute expectation of the convection equation. The Fourier transformed convection equation is:

$$
\operatorname{la} \Psi+V(x) \frac{d \Psi}{d x}=0
$$

where

$$
\Psi(x, a)=\frac{1}{\sqrt{2 \pi}} \int_{-\infty}^{\infty} C(x, t) e^{-t a t} d t
$$

Assuming the boundary condition, $C(0, t)=f(t)$, the solution of Eq. 98 is:

$$
\Psi(x, \alpha)=h(\alpha) e^{-i \alpha} \int_{0}^{x} d y / V(y)
$$

where $h(a)$ is the Fourier transform of $f(t)$. The travel time is assumed to have the following statistical properties:

$$
T(x)=\int_{0}^{x} \frac{d y}{V(y)}=\int_{0}^{x} \frac{d y}{u(y)}+\Delta(x)
$$

where $d \Delta / d x=w(x)$ is a purely random, stationary Gaussian process, and $u(x)$ is a mean reciprocal velocity: $1 / u(x)=\langle 1 / V(x)\rangle$. Taylor's theorem can be used to clarify the behavior of $\Delta(x):\langle\Delta(x)\rangle=0$, and 


$$
\frac{d}{d x}\left\langle\Delta^{2}\right\rangle=2 \int_{0}^{x}\langle w(x) w(y)\rangle d y=2 \lambda
$$

where $\lambda$ is a constant. Therefore,

$$
\left\langle\Delta^{2}(x)\right\rangle=2 \lambda x
$$

Applying Eq. 97 to Eq. 99, using Eq. 100, and

$$
\Delta(x)=\int_{0}^{x} w(y) d y
$$

gives:

$$
\langle\Psi(x, \alpha)\rangle=h(\alpha) \exp \left(-i \alpha \int_{0}^{x} d y / u(y)-\lambda \alpha^{2} x\right) .
$$

Hence, the expectation satisfies the equation:

$$
\frac{d}{d x}\langle\Psi\rangle+\left(i \alpha / u(x)+\lambda \alpha^{2}\right)\langle\Psi\rangle=0
$$

Inverting the Fourier transform for Equations 102 and 103 yields, respectively:

$$
\langle C(x, t)\rangle=\int_{-\infty}^{\infty} f(r) G(x, t-\gamma) d_{\gamma}
$$

where

$$
G(x, t)=\frac{1}{\sqrt{2 \pi \sigma}} \exp \left[-\frac{(t-T)^{2}}{2 \sigma^{2}}\right]
$$


with

$$
\bar{T}(x)=\int_{0}^{x} d y / u(y), \sigma^{2}=2 \lambda x,
$$

and

$$
\frac{\partial}{\partial t}\langle c\rangle+u(x) \frac{\partial}{\partial x}\langle c\rangle=u(x) \lambda \frac{\partial^{2}}{\partial t^{2}}\langle c\rangle .
$$

In view of the transport equations form, Eq. 106, is clearly non-Fickian. Evidently the statistical assumptions for Eq. 100 imply that travel time is Gaussian distributed according to Eq. 105.

Now, consider, instead, the case that:

$$
V(x)=u(x)+w(x)
$$

The calculation of expectation is restricted to small variations of $w(x)$ so that:

$$
\frac{1}{V}=\frac{1}{u} \frac{1}{1+w / u}=\frac{1}{u}\left(1 \frac{w}{u}+\varepsilon\right)
$$

where $\varepsilon=O\left[(w / u)^{2}\right]$. Then

$$
T(x)=T(x)-A(x)
$$

where

$$
T(x)=\int_{0}^{x}(1+\varepsilon) d y / u(y)
$$


and

$$
A(x)=\int_{0}^{x} w(y) d y / u^{2}(y)
$$

It can be proven (Chandrasehar in Simpson 1979) that Eq. 110 is also Gaussian distributed with $\langle A(x)\rangle=0$, and

$$
\left\langle A^{2}(x)\right\rangle=2 \lambda \int_{0}^{x} d y / u^{4}(y)
$$

(See p. 23 of N. Wax 1954.) Then, the expectation $\langle e i a A(x)>$ is the characteristic function (moment generating function) given by:

$$
\left\langle e^{i a A(x)}>=e^{-a^{2}<A^{2}(x)>/ 2}\right.
$$

Applying Equations 108 and 112 to the solution (Eq. 99) yields:

$$
\langle\Psi(x, a)\rangle=h(\alpha) \exp \left(-i \alpha \bar{T}(x)-\frac{a^{2}}{2}\left\langle A^{2}(x)\right\rangle\right) \text {. }
$$

And the expected concentration is again given by Equations 104 and 105, with mean (Eq. 109) and variance, $\lambda^{2}=\left\langle A^{2}(x)\right\rangle$. The transport equation is similar to Eq. 106, and given by:

$$
(1+\varepsilon) \frac{\partial}{\partial t}\langle c\rangle+u(x) \frac{\partial}{\partial x}\langle c\rangle=\frac{\lambda}{u^{3}(x)} \frac{\partial^{2}}{\partial t^{2}}\langle c\rangle .
$$




\section{Fickian Transport:}

It can be demonstrated that Fickian transport is a consequence of velocity variations which are uncorrelated in time. Thus, suppose that

$$
v(t)=u(t)+w(t)
$$

where $w(t)$ represents a purely random, stationary, Gaussian process. Assuming the boundary condition $c( \pm \infty, t)=0$ and deterministic initial condition $c(x, 0)=c_{0}(x)$, Fourier transforming the convection equation with respect to $x$ gives

$$
\frac{d \Psi}{d t}+i u(t) y \Psi=0
$$

The solution of Eq.116 is

$$
\Psi(y, t)=\Psi_{0}(y) e^{-i y} \int_{0}^{t} V(\gamma) d \gamma
$$

where $\Psi_{0}(y)$ is the transform of the initial distribution. Applying theorem (Eq. 97), but with $t$ replacing $x$, the expectation of Eq. 117 is:

$$
\langle\Psi(y, t)\rangle=\Psi_{0}(y) \exp \left(-i \int_{0}^{t} u(\gamma) d \gamma-\lambda y^{2} t\right) .
$$

Then Eq. 118 satisfies

$$
\frac{d}{d t}\langle\Psi\rangle+\left(\text { iyu }(t)+\lambda y^{2}\right)\langle\Psi\rangle=0
$$


Inverting the Fourier transforms for Equations 118 and 119 while utilizing the Fourier convolution theorem yields

$$
\langle C(x, t)\rangle=\int_{-\infty}^{\infty} C_{0}(y) G(x-y, t) d y
$$

where now

$$
G(x, t)=\frac{1}{\sqrt{4 \pi \lambda t}} \exp \left[-\frac{x-\int_{0}^{t} u(\gamma), d \gamma^{2}}{4 \lambda t}\right],
$$

and the classical convective-diffusion equation is obtained as

$$
\frac{\partial}{\partial t}\langle c\rangle+u(t) \frac{\partial}{\partial x}\langle c\rangle=\lambda \frac{\partial^{2}}{\partial x^{2}}\langle c\rangle
$$

where $\operatorname{Var}[\mathrm{V}]=2 \lambda$.

\section{Ensemble Statistical Moments}

So far the discussion of ensembles has been concerned only with the calculation of expectations of transport quantities. The principal advantage of the method, however, is that complete statistical information is contained in the velocity ensemble's probability distribution. Indeed, the quantities concentration and flux obtained as solutions of the convection equation constitute transformations of a velocity ensemble $\varepsilon$. Thus, those quantities are also characterized by probability distributions. For example, the cumulative distribution function for concentration is represented formally by:

$$
q(C ; x, t)=\int_{\varepsilon} \theta[C-C(x, t ; \vec{v})] d P[\vec{v} ; x, t]
$$


where $c(x, t ; v)$ is an explicit integral of the convection equation which defines a functional on the velocity fields $v(x, t)$, and $\theta(x)$ denotes the characteristic function equal 1 if $x>0$, equal zero otherwise. The distribution function (Eq. 123) represents the probability that the concentration takes values less than $c$, and it equals the probability weight of the velocity set in $\mathcal{E}$ satisfying that condition. Statistical moments of $n$ 'th order are defined as $(n=1,2, \ldots)$

$$
\begin{aligned}
\left\langle c^{n}\right\rangle(x, t) & =\int_{0}^{\infty} c^{n} d q(c ; x, t) \\
& =\int_{e} c^{n}(x, t ; v) d P[\vec{v} ; x, t]
\end{aligned}
$$

A statistical fact is that the probability distribution (Eq. 123) is completely specified by the entire set of moments (Eq. 124). Therefore, a distribution (Eq. 123) for the concentration can be uniquely specified only if al1 moments can be measured or estimated. On the other hand, it can be shown for some ensemble examples that a completely specified distribution (Eq. 123) determines uniquely the velocity probability distribution. This result indicates an important aspect of the ensemble method: the velocity probability distribution cannot in general be uniquely inferred indirectly from an incomplete estimation of statistical moments of transport quantities. However, that aspect allows for adequate description of a transport system by different approximate velocity distributions, in as much as the statistics of transport quantities are usually not completely available anyway.

At this point in the ensemble discussion, it is important to emphasize two conceptual origins of a velocity ensemble. In the first, velocity variation is viewed as a result of macroscopic variations occurring within a megascopic measurement volume (or as microscopic variation within a macroscopie volume). From an experimentalist viewpoint, velocity variation within a megascopic sample is stochastic, and in principle it is possible to measure the actual velocity probability distribution. That is, the velocity variation is observable. It would be feasible to infere from a solute tracer 
experiment the velocity distribution by measuring concentration variation within a megascopic sample.2 Such measurement detail would serve to estimate the actual concentration distribution (Eq. 1.23). However, detailed macroscopic mesurement is usually impractical or impossible. But if only the mean and variance of concentration were required, for example, then many different velocity distributions could provide a sufficient description of transport. Then in applying the ensemble method with a particular velocity probability distribution there is no assurance that $\mathrm{Eq} .124$ represents the moments beyond the expectation in the actual system, unless the velocity probability distribution is obtained by direct measurement.

In the second conceptualization, the velocity ensemble represents the multitude of possible streamlines allowed by measurement uncertainty in the hydrologic flow properties. The expectation of concentration represents the mean of all possible descriptions of the unknown concentration in the actual system. There is no physical reason that this mean should coincide with the actual concentration. Deviation of the expected concentration from the various descriptions based on convective transport associated with ensemble members does not necessarily represent actual dispersion in the hydrologic system, either.

It represents only the uncertainty in identifying the actual concentration. In this case, the moments determine the statistical nature and magnitude of that uncertainty. Now if, however, the uncertainty in hydrologic properties (potential, conductivity, and porosity) reflects the actual spatial variability inherent at the macroscopic scale, then the ensemble may be able to describe the actual dispersion. This can be verified only by experiment, however.

As a final point, it must be emphasized that the concept of the three scales of measurement, which are micro-, macro-, and mega-scopic, is a synthetic construct. The essential concept is that uncertain variations of water velocity at a volume scale below the sample volume used to represent points in a hydrologic system are inevitably responsible for unexplained dispersion phenomena. In order to properly describe transport, an ensemble must include that uncertainty in water velocity. Indeed, although the mass 
balance of water flow in a steady-state system is adequately described by mean velocity, an advancing solute front will distringuish the velocity variation, which must be accounted for in a correct transport prediction.

Ensemb le Addition

An ensemble method based on the convection equation alone has been demonstrated. However, there is no fundamental mathematical restriction on including a diffusion or macroscopic dispersion coefficient. Alternatively, the convective-dispersion Equation 1 in Chapter 1 can be viewed as stochastic and used as the fundamental transport equation. The convection equation is used because it simplified the presentation, and constitutes a more fundamental basis. Indeed, when the macroscopic coefficient $D$ is representable by an equivalent ensemble the transport is still described by a stochastic convection equation, by adding that ensemble to the ensemble for megascopic velocity variation.

The concept of ensemble addition will be demonstrated first for the simple one-dimensional, constant velocity ensemble. Let the Gaussian velocity p.d.f (Eq. 37) with mean $\bar{V}_{1}$, and variation $\mu_{1}^{2}=2 \mathrm{D}_{1} /$ t represent the macroscopic dispersion characterized by $D_{1}$ (constant). Megascopic variations are represented by some p.d.f., $p_{2}\left(v_{2}: x, t\right)$. Adding the two ensembles amounts to defining the total stochastic velocity as $V=V_{1}+V_{2}$ and compounding the velocity probabilities. The complete velocity p.d.f. is given by

$$
\begin{aligned}
p(v ; x, t) & =\iint \delta\left(V_{1}+V_{2}-V\right) p_{1}\left(V_{1} ; t\right) p_{2}\left(V_{2} ; x, t\right) d V_{1} d V_{2} \\
& =\int_{-\infty}^{\infty} p_{1}\left(V-v_{2} ; t\right) p_{2}\left(V_{2} ; x, t\right) d V_{2} .
\end{aligned}
$$


The p.d.f. (Eq. 125) is a convolution integral. Consider now an initial value formulation (Eq. 32). A stochastic integral of the convective-dispersion Eq. 34 with $u=\bar{V}_{1}+v_{2}$ and $D=D_{2}$ is

$$
\begin{aligned}
\bar{C}\left(x, t ; v_{2}\right) & =\int_{-\infty}^{\infty} C_{0}(y) g(x-y, t) d y \\
& =\int_{-\infty}^{\infty} C_{0}\left[x-\left(v_{1}+v_{2}\right) t\right] p_{1}\left(v_{1} ; t\right) d v_{1}
\end{aligned}
$$

with $g(x, t)$ given by Eq. 48. Expectation of Eq. 125 is

$$
\begin{aligned}
\bar{C}(x, t) & =\int_{-\infty}^{\infty} \bar{C}\left(x, t ; v_{2}\right) p_{2}\left(V_{2} ; x, t\right) d V_{2} \\
& =\int_{-\infty}^{\infty} C_{0}(x-V t) p(V ; x, t) d V .
\end{aligned}
$$

Therefore, the expectations of the convection and convective-dispersion equation solutions are identical. In the special case that $p_{2}$ is also Gaussian with mean $\bar{V}_{2}$ and variance $\mu_{2}^{2}=2 \mathrm{D}_{2} / \mathrm{t}$, then Eq. 125 is again a Gaussian Eq. 37 with $\bar{V}=\bar{V}_{1}+\bar{V}_{2}$ and $D=D_{1}+D_{2}$.

The more general constant velocity case is considered next. Assume that Eq. 34 has an equivalent ensemble described by $p_{1}\left(v / u_{1} ; x, t\right)$, a velocity p.d.f. conditional on $u_{1}$. Then

$$
\bar{c}\left(x, t ; u_{1}\right)=\int_{-\infty}^{\infty} c(x, t ; v) p_{1}\left(v / u_{1} ; x, t\right) d v
$$


is a solution of Eq. 34 for some integral of the convection equation. The expectation of Eq. 128 for $u_{1}=\nabla_{1}+V_{2}$ is given by Eq. 30 where

$$
p(v ; x, t)=\int_{-\infty}^{\infty} P_{1}\left(v \mid \bar{v}_{1}+v_{2} ; x, t\right) p_{2}\left(V_{2} ; x, t\right) d V_{2}
$$

If $p_{1}$ satisfies the condition

$$
p_{1}\left(v \mid \bar{v}_{1}+v_{2} ; x, t\right)=p_{1}\left(v-v_{2} \mid \nabla_{1} ; x, t\right)
$$

then the complete p.d.f. is given by a convolution (Eq. 125), otherwise it may not be. Example ensembles (Eq. 37), (Eq. 53), and (Eq. 57) satisfy Eq. 130, but Eq. 66 does not.

In the still more general situation for which velocity depends on location, the problem of constructing the complete velocity distribution is unsolved. A velocity probability distribution can at present be expressed only formally, since existence will depend on the specific representation of the velocity ensemble. To clarify the difficulties, a formal development is presented. The stochastic concentration integrals $\vec{c}(x, t ; u)$, which are functionals of the velocity $u(x)$, satisfy Eq. 34 . It is sufficient also to be concerned with only the case that $D$ is constant, in view of experimental information on macroscopic dispersion. For an initlal value formulation the solutions are

$$
\tau(x, t ; u)=\int c_{0}(y) g(x-y, t ; u) d y
$$

where $g(x, t ; u)$ is the fundamental solution of Eq. 34 with $g(x, 0: u)=\delta(x)$, which by solute conservation determines a p.d.f. in $x$. A constant velocity p.d.f. yielding an equivalent ensemble is given by

$$
p_{1}\left(v / u_{1} ; t\right)=\operatorname{tg}\left(v t, t ; u_{1}\right)
$$


Letting $\mathrm{P}_{2}[\mathrm{u} ; \mathrm{x}]$ represent a stationary probability distribution for the ensemble $\varepsilon_{\text {of }} u(x)$, the formal complete velocity p.d.f. expression is:

$$
p(V ; x, t)=\int_{\varepsilon} p_{1}(V \mid u ; t) d P_{2}[u ; x] .
$$

Clearly the difficulties are: the explicit behavior and domain of the functional $p_{1}(v / u ; t)$ of $u$ must be known, existence of the expectation (133) depends on the definitions of and $p_{2}[u ; x]$, and Eq. 132 must be defined on some averageable (measurable) subset of $\boldsymbol{E}$. Difficulty with the case of a boundary value formulation is even more severe because it is not known for which $u(x)$ the Green's function for solutions of Eq. 34 defines a p.d.f. in travel time as in Eq. 45. These difficulties remain as open research questions.

\section{TRAVEL TIME ENSEMBLES}

Definition

A particularly useful yet mathematically simple ensemble is obtained from a travel time formulation, because solutions (integrals) of the convection equation can be expressed explicitly as functions of the solute travel time between the release and observation points. These ensembles provide a precise description of independent flow tube models of transport, such as occurs in an idealized layered system. But they are not restricted to such models, and generally encompass transport problems with spatially varying velocity. Development of a travel time ensemble was suggested in the previous discussions. Here the approach is developed in more detail with applications into consideration.

Let $\varepsilon$ denote an ensemble of stochastic velocities $V(x)$ with a probability distribution $P[V ; x]$, representing a stationary system. (Note that the general 
case including a time dependence is not excluded by the method.) The travel time between $x_{0}$ and $x$ for each velocity is:

$$
T\left(x ; x_{0}\right)=\int_{x_{0}}^{x} d y / V(y)
$$

And Eq. 134 determines a new stochastic variable. It is required that the ensemble determines a well-defined travel time P.d.f. given by

$$
p\left(t ; x, x_{0}\right)=\int_{e^{0}} \delta\left(t-T\left(x ; x_{0}\right)\right) d P[V ; x]
$$

where the integral

$$
\int_{0}^{t} p\left(r ; x, x_{0}\right) d r
$$

equals the probability of travel times less than $t$ when observed at $x$. For each averageable function of travel time (expectation is defined) $f\left(T\left(x ; x_{0}\right)\right)$, the expectation is given by

$$
\begin{aligned}
\left\langle f\left(T\left(x ; x_{0}\right)\right)\right\rangle & =\int_{e} f\left(T\left(x ; x_{0}\right)\right) d P[V ; x] \\
& =\int_{0}^{\infty} f(t) p\left(t ; x, x_{0}\right) d t .
\end{aligned}
$$

Notice that generally there is no reason to exclude negative travel times in Eq. 136 . 
In the cases that the boundary values of concentration in solution or solute flux Eq. 7 are deterministic and specified by Eq. 42, then the observed expectations are given by the same expression:

$$
\begin{aligned}
& \text { (i) } \bar{C}\left(x, t ; x_{0}\right)=\int_{0}^{\infty} f(t-\gamma) P\left(\gamma ; x_{2} x_{0}\right) d \gamma \\
& \text { (i i) } U\left(x, t ; x_{0}\right)=
\end{aligned}
$$

Expectation (Eq. 137) is obtained by applying Eq. 136 to the solutions (Eq. 20) and (Eq. 22), respectively. Of course, the units of $f$ differ for each case. As before, $f(t)=0$ if $t<0$, and $f$ may also depend on $x_{0}$. To simplify notation, $x_{0}$ equals zero when it does not appear explicitly.

Existence of an equivalent convective-dispersion system has an immediate characterization in terms of the travel time p.d.f. An equivalent convective-dispersive system with time independent velocity $u(x)$ and dispersion coefficient $D(x)$ exists if the expectations (Eq. 137) satisfy

$$
\begin{aligned}
& \text { (i) } \frac{\partial \bar{C}}{\partial t}+u \frac{\partial \bar{C}}{\partial x}=\frac{1}{\bar{\phi}} \frac{\partial}{\partial x}\left(\bar{\phi} D \frac{\partial C}{\partial x}\right) \\
& \text { (ii) } \bar{U}=u_{p}-D \frac{\partial \bar{\rho}}{\partial x}
\end{aligned}
$$

for some $\rho$ such that

$$
\frac{\partial \bar{\rho}}{\partial t}+\frac{\partial \bar{U}}{\partial x}=0
$$


in each case. Note that the definition of $D$ is different in each case, and $\bar{\phi}$ is the observed porosity satisfying

$$
\frac{\partial \bar{\phi}}{\partial t}+\frac{\partial}{\partial x}(\bar{\phi} u)=0
$$

The constraint (Eq. 140) is required for Eq. 138 (i) if the flow system is to be conservative. In Eq. 138 ( $i i$ ) the observed flux must then satisfy a convective-dispersion type equation given by

$$
\frac{\partial \bar{U}}{\partial t}+u \frac{\partial \bar{U}}{\partial x}=D \frac{\partial^{2} \bar{U}}{\partial x^{2}}
$$

Therefore, necessary conditions for the existence of an equivalent convective-dispersive system holding for all boundary values $f$ are that the travel time p.d.f. (135) for each case satisfy

$$
\begin{aligned}
& \text { (i) } \frac{\partial p}{\partial t}+u \frac{\partial p}{\partial x}=\frac{1}{\bar{\phi}} \frac{\partial}{\partial x} \quad\left(\bar{\phi} D \frac{\partial p}{\partial x}\right) \\
& \text { (ii) } \frac{\partial p}{\partial t}+u \frac{\partial p}{\partial x}=D \frac{\partial^{2} p}{\partial x^{2}}
\end{aligned}
$$

for $x>x_{0}$. The condition $p(0+; x)=0$ for $x>x_{0}$ was used in (142). Requiring in additon the property

$$
P\left(t ; x_{0}, x_{0}\right)=\delta(t),
$$


Eq. 142 ( $i)$ is also a sufficient condition. To obtain sufficient conditions for Eq. 138 ( $i i)$, let $G(x, t)$ (depending on $x_{0}$ ) determine a solution of the convective-dispersion equation for some $u(x)$ and $D(x)$ by

$$
\bar{\rho}\left(x, t ; x_{0}\right)=\int_{0}^{\infty} f(\gamma) G(x, t-\gamma) d_{\gamma}
$$

such that $G(x, 0)=0\left(x>x_{0}\right)$ and

$$
\left(u G(x, t)-D \frac{\partial G}{\partial x}(x, t)\right) \quad x=x_{0}=\delta(t) \text {. }
$$

Suppose that

$$
p\left(\gamma: x, x_{0}\right)=u(x) G(x, \gamma)-D(x) \frac{\partial G}{\partial x}(x, \gamma)
$$

defines a p.d.f. in $\gamma>0$. Then Eq. 144 determines an equivalent system satisfying Eq. 137 (ii).

In the general case, irrespective of an equivalent convective-dispersive system, an observed (average) concentration $\bar{f}$ conjugate to the observed, expected flux under conservation must satisfy Eq. 139. Indeed, notice the $\overline{0}$ need not equal the expectation defined by

$$
\langle n\rangle(x, t)=\int_{e}^{f\left(t-T\left(x ; x_{0}\right)\right)} \frac{f(x)}{V(x[V ; x]} \text {. }
$$

Conversely, if the observed concentration $\bar{\rho}$ is its expectation (Eq. 147), then the observed flux which satisfies Eq. 139 may not equal the expectation (Eq. 137) (ii). Deterministic boundary conditions similar to Eq. 137 (i) could have been imposed on $\bar{\rho}$ also instead of $C$; and the same relationship 
between observed values would be required. Thus, in applications of the travel time ensemble a distinction between expected and observed values must usually be made.

In view of the constant velocity ensemble discussion, an interesting result becomes evident: every ensemble represented by a travel time p.d.f. has an equivalent constant velocity ensemble. The transformation $\gamma=x / V$ for constant $V>0$ establishes the relationship in the expectations (Eq. 137), so that the constant velocity p.d.f. is

$$
p *(v ; x)=x p(x / v ; x) / v^{2} \quad(x>0)
$$

The result remains true even if the travel time p.d.f depends explicitly on $t$. This result clarifys the concept that a constant velocity system with location and time dependent velocity pd.f. actually represents a spatially dependent velocity ensemble. Moreover, the conclusion remains valid for an initial value formulation as well. In particular, the constant velocity ensembles previously discussed yield examples of travel time ensembles. 'The p.d.f. given by

$$
p(\gamma ; x)=\frac{1}{\sqrt{\pi a}} \frac{e^{-(\gamma-x / u)^{2} / a \gamma}}{\sqrt{\gamma}} \quad(\gamma>0)
$$

with $\mathrm{a}=40 / \mathrm{u}^{2}$ for constant $\mathrm{u}$ and $\mathrm{D}$ provides an example described by an equivalent convective-dispersive system. The corresponding constant velocity p.d.f. is given by Eq. 53 .

Moment Generating Functions

The ensemble expectation (Eq. 137) for the boundary value formulation can be found explicitly in terms of statistical cumulants (semi-invariants) 
associated with a travel time probability density. The moment generating function for a particular p.d.f. is defined by

$$
\begin{aligned}
M(s ; x) & =\int_{0}^{\infty} e^{s \gamma} p(r ; x) d \gamma \\
& =\sum_{n=0}^{\infty}\left\langle T^{n}(x)>\frac{s^{n}}{n !} .\right.
\end{aligned}
$$

Expressed in terms of the statistical cumulants of travel time to location $x$ denoted by $\beta_{k}(x)$, the moment generating function is given by

$$
M(s ; x)=\exp \left(\sum_{k=0}^{\infty} \beta_{k}(x) \frac{s^{k}}{k !}\right) .
$$

For example, $\beta_{0}=0, \beta_{1}=E[T(x)]$, and $\beta_{2}=\operatorname{Var}[T(x)]$ are the first three cumulants. A Gaussian distribution is characterized as having all higher order $(k>2)$ cumulants equal to zero.

Now letting $\psi(x, s)$ denote the Laplace transform with respect to $t$ of either the stochastic concentration or flux, for a boundary value formulation the transform is

$$
\psi(x, s)=h(s) e^{-s T(x)}
$$

where $h(s)$ is the transform of $f(t)$. Then for a time independent p.d.f. Eq. 135, the expectation of Eq. 152 is the Laplace transform of the expectation Eq. 137, which is given by

$$
\begin{aligned}
\bar{\psi}(x, s) & \equiv h(s) \int_{\varepsilon} e^{-s T(x)} d P[V ; x] \\
& =h(s) M(-s ; x) .
\end{aligned}
$$


Equation 153 indicates that the statistical behavior of the travel time p.d.f. is derived from the time dependence behavior of the expectations. In particular, the cumulants are obtained from the generating function as follows:

$$
\beta_{k}=\left.(-1)^{k} \frac{d^{k}}{d s^{k}} \ln M(-s ; x)\right|_{s=0}
$$

Thus, the travel time p.d.f. can be deduced directly from a tracer experiment if the expectation of a transport quantity is assumed equal to its observed value.

If negative travel times are allowed by including negative velocities in an ensemble, the case with an approximate Gaussian travel time distribution can be considered. The moment generating function in this case is

$$
M(s ; x)=\exp \left\{\bar{T}(x) s+\sigma^{2}(x) \frac{s^{2}}{2}\right\}
$$

where

$$
T(x)=\int_{0}^{x} d y / u(y) \quad \text { (positive) }
$$

and

$$
\sigma^{2}(x)=\left\langle\Delta^{2}(x)\right\rangle
$$

for a travel time variation $\Delta(x)$ defined by

$$
T(x)=\bar{T}(x)+\Delta(x)
$$

If $\mathrm{d} \Delta / \mathrm{dx}$ is a purely random, stationary, Gaussian process (Eq. 96), then $\sigma^{2}$ $=2 \lambda x$, and the travel time p.d.f. is given by Eq. 105 for this special case. 
When the variation $\Delta$ is sufficiently small relative to the mean $T$, negative travel times enter only with arbitarily small probability.

It can be demonstrated that the covariance behavior of $V(x)$ is included in the travel time variance as follows. A difference is defined by

$$
w(x)=\frac{1}{v(x)}-\frac{1}{u(x)}
$$

so that

$$
\Delta(x)=\int_{0}^{x} w(y) d y
$$

with

$$
\langle\Delta(x)>=0
$$

Then

$$
\sigma^{2}(x)=\int_{0}^{x} \int_{0}^{x}\langle w(y) w(z)\rangle d y d z
$$

where

$$
\langle w(y) w(z)\rangle=\operatorname{Cov}[1 / V(y), 1 / V(z)]
$$

If the difference $w(x)$ is a stationary process, then an autocovariance function exists defined by

$$
A(y)=\langle w(x) w(x+y)\rangle
$$


independent of $x$. The variance (Eq. 157) is then

$$
\sigma^{2}(x)=2 \int_{0}^{x}(x-y) A(y) d y \quad
$$

Small velocity variations case:

Suppose now that

$$
V(x)=u(x)+w(x)
$$

where the velocity variation $w$ is small relative to the mean $u$. Using

$$
\frac{1}{V}=\frac{1}{u}\left(1-\frac{W}{u}+c\right),
$$

the variance in travel time is:

$$
\sigma^{2}(x)=\int_{0}^{x} \int_{0}^{x} \frac{\langle w(y) w(z)\rangle}{(u(y) u(z))^{2}} d y d z .
$$

Using a second order expansion,

$$
\frac{1}{v}=\frac{1}{u}\left(1-\frac{w}{u}+\frac{w^{2}}{u^{2}}+\varepsilon\right),
$$

the expectation of travel time is:

$$
\langle T(x)\rangle=T(x)+\int_{0}^{x} \frac{\left\langle w^{2}(y)\right\rangle}{u^{3}(y)} d y .
$$


Then by retaining terms only up to second order, an expansion of the stochastic concentration about $\lambda=t-\bar{T}(x)$ yields an expected concentration given by

$$
\bar{c}(x, t)=f(\lambda)-f^{\prime}(\lambda) \int_{0}^{x} \frac{\left\langle w^{2}(y)\right\rangle}{u^{3}(y)} d y+f^{\prime \prime}(\lambda) \frac{\sigma^{2}}{2}(x) .
$$

The same equation holds for flux (Eq. 137) ( $i i)$. This shows that expectations (Eq. 137) are essentially determined by a Gaussian travel time distribution, provided the velocity variation is sufficiently small.

Initial Condition Formulation

Although an initial conditions formulation of stochastic transport is perhaps not as relevant to a contaminant release analysis as the boundary condition case, a study of this case nevertheless provides some further insights into the velocity ensemble concept. The discussion will be restricted to just the stochastic concentration $c(x, t ; V)$ satisfying the convection equation:

$$
\frac{\partial C}{\partial t}+V(x) \frac{\partial C}{\partial x}=0
$$

Stochastic convective flux satisfies an identical equation (Eq. 16), and so the same formulation will apply to flux. Now provided that the inverse of the travel time function (Eq. 134) exists, as denoted by $T-1$, the stochastic concentration solutions of Eq. 165 are:

$$
c(x, t ; V)=c_{0}\left[T^{-1}\left(T\left(x ; x_{0}\right)-t\right)\right]
$$

for $x \geq x_{0}$ and $0 \leq t \leq T\left(x, x_{0}\right)$, with initial condition $c(x, 0 ; V)=c_{0}(x)$. If the ensemble consists of only non-negative continuous velocity functions $V(x)$, then Eq. 166 yields proper solutions, since the required travel time inverse exists. It is clear that Eq. 166 is not a simple fixed function of travel time, and the ensemble expectation cannot be expressed directly as an average 
over travel time as in the previous case (Eq. 137). Apparently a new stochastic function having units of length arises from this formulation, and it is given by

$$
L\left(x, t ; x_{0}\right)=T^{-1}\left(T\left(x ; x_{0}\right)-t\right)
$$

Now in view of the fact that an initial value formulation of convective-dispersive transport governed by Eq. 1 in Chapter 1 includes naturally the entire domain $-\infty<x<\infty$ and the possibility of flow in either direction as well, it is appropriate to extend the definition of the concentration solutions. By using either a Laplace or Fourier transform of Eq. 165 with respect to $t$, two alternative representations of solutions are obtained, which are, respectively,

$$
c(x, t ; V)=\int_{x_{0}}^{x} \frac{c_{0}(y)}{V(y)} \delta+(t-T(x ; y)) d y
$$

for $x \geq x_{0}$, and

$$
c(x, t ; V)=\int_{-\infty}^{\infty} \frac{C_{0}(y)}{V(y)} \delta(t-T(x ; y)) d y
$$

for $-\infty<x<\infty$. If a proper inverse travel time function exists, then Eq. 168 and Eq. 169 reduce to solutions of the form (Eq. 166). For a positive velocity, Eq. 169 includes also negative travel times (Eq. 134) when $x<x_{0}$, and velocities in either direction are allowed. Indeed, Eq. 169 represents an average over all possible initial locations. 
To contrast the different formulations, observe that the deterministic boundary value case has solutions given by

$$
\begin{aligned}
c(x, t ; V) & =f\left(t-T\left(x ; x_{0}\right)\right) \\
& =\int_{0}^{t} f(\gamma) \delta+\left(t-T\left(x: x_{0}\right)-\gamma\right) d \gamma .
\end{aligned}
$$

for $x \geq x_{0}$ and $t \geq T\left(x ; x_{0}\right)$.

A useful alternative characterization of an ensemble's statistical properties will now be described, which clarifies the origin of various probability density functions for the stochastic functions (Eq. 134) and (Eq. 167). It is required for a given ensemble $\mathcal{E}$ that there exist a positive, linear functinal $<$ defined on a certain collection of functionals of $V(x)$ in $\varepsilon$. Those functionals have their expected values defined and are called averageable. An indicator function $1 \mathcal{\&}$ defined on $\mathcal{E}$ for a subset $\mathbb{d}$ equals unity if $V(x)$ is in $\mathscr{L}$ and equals zero outside of $\mathscr{L}$. Moreover, it is required that

$$
\left\langle\mathrm{I}^{\circ}\right\rangle=1
$$

The collection of subsets $\mathbb{E}$ for which the expectation (average) of I exists are the measurable subsets having a defined probability given by

$$
P[\mathscr{d}]=\langle\underline{\mathscr{L}}\rangle
$$

Positivity of $\langle\cdot>$ insures that Eq. 171 satisfies the probability condition:

$$
0 \leq P[\mathscr{Q}] \leq 1
$$

If $\mathcal{L}$ and $\boldsymbol{D}$ are disjoint measurable subsets of $\mathbb{E}$ then

$$
I_{D U} \mathcal{L}=I_{D}+I_{\mathscr{L}}
$$


and the additivity property

$$
P[D \cup \mathcal{d}]=P[D]+P[\mathscr{d}]
$$

follows from linearity. (See F. Riesz and B. Sz.-Nagz. 1965. for a mathematically precise development of positive, linear functionals and integration.) We shall proceed here formally and assume the validity of interchange of mathematical operations and existence of integrals.

A travel time description of an ensemble (Eq. 135) then follows from the definition

$$
\left.p\left(r ; x, x_{0}\right)=\frac{d}{d r}<\theta\left(r-T\left(x ; x_{0}\right)\right)\right\rangle,
$$

by taking the expectation of Eq. 170. The expectation indicated in Eq. 172 is that for the set of all velocities in $E$ such that $T\left(x ; x_{0}\right)<\gamma$. And the probability of those sets for all $r \geq 0$ must be determined by the expectation. A probability density for the stochastic function (Eq. 167) is obtained in a similar way by calculating the expectation of Eq. 166. First consider the physical meaning of Eq. 167. Assuming a positive velocity, for $t \leq T\left(x ; x_{0}\right)$ there exists a $y$ between $x_{0}$ and $x$ such that $t=T(x ; y)$, or $T\left(y ; x_{0}\right)=$ $T\left(x ; x_{0}\right)-t$. Therefore, Eq. 167 equals this $y$, which is the initial location of concentration observed at location $x$ and time $t$, having traveled $a$ distance $x-y$ forward. Thus $x-L\left(x, t ; x_{0}\right)$ is identifiable as a stochastic travel distance. Now the solution (Eq. 166) can be expressed as

$$
c(x, t ; v)=\int_{-\infty}^{\infty} c_{0}(y) \delta\left(y-L\left(x, t ; x_{0}\right)\right) d y
$$

Then, letting

$$
p\left(y ; x, t, x_{0}\right)=\frac{d}{d y}\left\langle\theta\left(y-L\left(x, t ; x_{0}\right)\right)\right\rangle
$$


determines the expected concentration as given by

$$
\langle c\rangle(x, t)=\int_{-\infty}^{\infty} c_{0}(y) p\left(y ; x, t, x_{0}\right) d y .
$$

The p.d.f. (Eq. 174) can be obtained easily for the constant velocity ensemble case. For a constant velocity $V$,

$$
L\left(x, t ; x_{0}\right)=x-v t
$$

and

$$
p(y ; x, t)=\langle\delta[y-(x-V t)]\rangle \text {. }
$$

This is the result obtained in Eq. 32 .

Conditions for the existence of an equivalent convective-dispersive system are easily obtained for Eq. 175. Such a system exists if and only if Eq. 174 itself satisfies Eq. 138 (i) for all y. In the case that $u$ and $D$ are constants, a p.d.f. (Eq. 174) determining an equivalent ensemble is

$$
p(y ; x, t)=g(x-y, t)
$$

for Eq. 48 .

Connection between Travel Time and Distance:

The extended solution can be used also to define a travel distance p.d.f. given by

$$
\begin{aligned}
p(y ; x, t) & =\langle\delta(t-T(x ; y)) / V(y)\rangle \\
& =\frac{d}{d y} P(y ; x, t)
\end{aligned}
$$


where

$$
P(y ; x, t)=\langle\theta(t-T(x ; y))\rangle
$$

is the cumulative probability function. Expectation of concentration is given by Eq. 175, independent of $x_{0}$. In view of the definition (Eq. 172), the relationship between the probability density functions is:

$$
P(y ; x, t)=\int_{-\infty}^{t} p(r ; x, y) d \gamma
$$

or

$$
p(\gamma ; x, y)=\frac{d}{d \gamma} P(y ; x, \gamma)
$$

Apparently, Eq. 177 determines a cumulative probability function for both $\gamma$ and $y$.

A Fourier transformation of the concentration provides an important relationship between the expectation ( $\mathrm{Eq} .175)$ and the characteristic function of travel time, which is defined as

$$
M(r ; x, y)=\int_{-\infty}^{\infty} e^{i r \gamma} p(r ; x, y) d \gamma .
$$

Let

$$
\begin{aligned}
\boldsymbol{\psi}(x, r ; v) & =\int_{-\infty}^{\infty} d t e^{i r t} c(x, t ; v) \\
& =\int_{-\infty}^{\infty} \frac{c_{0}(y)}{V(y)} e^{i r T(x, y)} d y .
\end{aligned}
$$


Then

$$
\begin{aligned}
\langle\boldsymbol{\psi}\rangle(x, r) & =\int_{-\infty}^{\infty} \frac{C_{0}(y)}{V(y)} M(r ; x, y) d y \\
& =\int_{-\infty}^{\infty} \frac{M(r ; x, y)}{i r} d c_{0}(y),
\end{aligned}
$$

since

$$
\psi(x, r ; V)=\int_{-\infty}^{\infty} \frac{e^{i r T(x, y)}}{i r} d c_{0}(y)
$$

A statistical fact is that the characteristic function (Eq. 180) is given by

$$
M(r ; x, y)=\exp \left\{\sum_{k=1}^{\infty} \beta_{k}(x, y) \frac{(i r)^{k}}{k !}\right\}
$$

where $\beta_{k}$ are the semi-invariants of the travel time between $y$ and $x$.

By substituting Eq. 179 into Eq. 180, using Eq. 176, and inverting the Fourier transform, the travel distance p.d.f. is found to be related to the characteristic function as follows:

$$
p(y ; x, t)=\frac{i}{2 \pi} \int_{-\infty}^{\infty} d r \frac{e^{-i r t}}{r} \frac{\partial}{\partial y} M(r ; x, y)
$$

The characteristic function can be expressed also in terms of the statistical moments of travel time, and they could be used in Eq. 184 instead.

It is a simple observation based on the expectation's representation Eq. 175 with Eq. 176 that every initial value formulation has an equivalent 
constant velocity ensemble. Indeed, the substitution of $y=x-V t$ into $E q .175$ for constant $V$ and the definition

$$
p^{\star}(V ; x, t)=t p(x-V t ; x, t) \quad(t>0)
$$

yields the result, since then

$$
\langle c\rangle(x, t)=\int_{-\infty}^{\infty} c_{0}(x-V t) p *(V ; x, t) d V \quad
$$

Spatially Stationary Ensembles:

A common problem confronted in the realm of theoretical transport methods is the prediction of future solute movement from past measurements of the concentration's location. Clearly, the history of solute movement in a particular region will not predict reliably the behavior within other regions, unless the water velocity's statistical properties are repeated in some physical sense. In general, the stochastic, convective transport description has shown that a complete statistical information over the region between initial location and observation is required. If the travel distance p.d.f. is spatially stationary, that is, depends only on the difference $x-y$, then the transport history, however, does determine future movement. The spatial stationarity property will depend, of course, on the velocity ensembles repetition over every interval $y$ to $x$ when translated in the direction of flow.

A constant velocity ensemble example serves to clarify explicitly this concept. Thus, suppose the ensemble is characterized by $p *(V ; t)$, independent of location. Calculation of Eq. 176 gives

$$
\begin{aligned}
p(y ; x, t) & =\int_{-\infty}^{\infty} \frac{\delta}{V}(t-(x-y) / V) p^{\star}(V ; t) d V \\
& =p^{\star}((x-y) / t ; t) / t \quad(t>0) .
\end{aligned}
$$


Then the expectation is:

$$
\langle c\rangle(x, t)=\int_{-\infty}^{\infty} c_{0}(x-y) p^{\star}(y / t ; t) d y / t .
$$

Since Eq. 187 is a convolution, a Fourier transformation with respect to $x$ will determine the characteristic function of the velocity p.d.f. as follows:

$$
\langle\psi\rangle(r, t)=\psi_{0}(r) M^{*}(r t ; t)
$$

where

$$
M \star(r ; t)=\int_{-\infty}^{\infty} d y e^{i r y} p^{\star}(y ; t)
$$

and $\langle\psi\rangle, \psi_{0}$ are the transforms of $\langle c\rangle, c_{0}$, respectively. Now if $p^{*}$ is independent of $t, a$ measurement of $c_{0}$ at $t=0$ and $\langle c\rangle$ at time $t$ will establish $M^{\star}(r)$ for all subsequent times, according to Eq. 188.

In the more general situation, the cumulative probability function (Eq. 178) is given by

$$
P(x-y ; t)=\int_{-\infty}^{t} p(\gamma ; x-y) d \gamma,
$$

since the travel time p.d.f. is spatially stationary. Now because Eq. 190 is a joint cumulative probablity for $x-y$ and $t$, it has the form

$$
P(x ; t)=\int_{-\infty}^{x} \int_{-\infty}^{t} g(y, r) d y d \gamma
$$

where $g(x, t)$ is a joint probability density. Hence, Eq. 179 is a marginal distribution given by 


$$
p(\gamma ; x-y)=\int_{-\infty}^{x-y} g(z, \gamma) d z
$$

Using Eq. 190 and Eq. 192, rate of change of expected concentration satisfies:

$$
\frac{\partial}{\partial t}\langle c\rangle=-\int_{-\infty}^{\infty} c_{0}(x-y) g(y, t) d y
$$

Defining the characteristic function for the $x$ variable by

$$
M(r ; t)=\int_{-\infty}^{\infty} d x e^{i r x} g(x, t)
$$

results in the equation

$$
\frac{\partial}{\partial t}\langle\psi\rangle=-\psi_{0}(r) M(r ; t)
$$

which determines the characteristic function (Eq. 194). If the functional form of the semi-invariant time dependence is known, then only as many concentration spatial distribution measurements need be obtained at different times as will suffice to determine the unknown parameters of the characteristic function. On the other hand, when the statistical moments of travel distance as functions of travel time are assumed known, then the inverse transform of Eq. 195 yields an evolution equation with right-hand side given by a linear differential operator acting on the initial concentration distribution.

The special case for which

$$
p(r ; x)=\left(x / \gamma^{2}\right) p *(x / \gamma)
$$


for some single variable p.d.f, $p^{*}$, corresponds to the equivalent constant velocity ensemble result (Eq. 187), since then

$$
\frac{\partial P}{\partial y}(x-y ; t)=p \star((x-y) / t) / t
$$

is the travel distance p.d.f.

Without providing specific details, the expected concentration can be obtained for the weaker second order stationarity case given by $T(x ; y)=$ $\bar{T}(x ; y)+\gamma$ with the travel time error distributed as in Eq. 192. Then the some what physically unreasonable assumption of a stationary expected travel time can be discarded.

\section{A Stochastic Flow Tube Approach}

A specific application of the stochastic convective transport method to a single radioactive species with sorbtion behavior is discussed in this section. Transport is visualized as occurring in an essentially one-dimensional system, composed of independent transport pathways having negligible exchange of solute mass between them. The idealized stratified system (Eq. 28) and a flow tube model of a ground water system are examples. An appropriate ensemble is defined by identifying each layer or flow tube with the solute velocity $V(x)$ within each member. In such examples, the ensemble members are each observable. The convective transport equation for a radioactive decaying solute is:

$$
K \frac{\partial \rho}{\partial t}+\frac{\partial}{\partial x}\left(V_{w} \rho\right)=-\lambda K \rho
$$

where $\lambda$ is the decay rate constant and $K(x)$ is the sorbtion retardation factor, possibly depending on location $x, v_{w}(x)$ is the water velocity in each tube. Note that $K_{\rho}$ is the total solute concentration in the porous medium, whereas $\rho$ is that actually dissolved into solution. Only the 
calculation of expected stochastic convective flux will be considered for a deterministic boundary value formulation. The convective flux, $U=V_{w} p$, satisfies

$$
\frac{\partial U}{\partial t}+V \frac{\partial U}{\partial x}=-\lambda U
$$

where now

$$
V(x)=V_{w}(x) / K(x)
$$

is the retarded velocity of solute movement. Since each solution of Eq. 197 has the form

$$
e^{-\lambda t} U(x, t)
$$

where $U$ is a solution for $\lambda=0$, it is sufficient to consider the transport without decay. Decay effects can be re-included by multiplying expected values by the decay factor as in Eq. 199. Assuming a release rate $J\left(t ; x_{0}\right)$ at $x=x_{0}$, the expected convective flux is:

$$
U(x, t)=e^{-\lambda t} \int_{-\infty}^{\infty} J\left(t-\gamma ; x_{0}\right) p\left(\gamma ; x, x_{0}\right) d \gamma,
$$

including the radioactive decay, and where the travel time p.d.f. is for the retarded solute velocity (Eq. 198). In case the retardation factor is everywhere constant, the expectation can be taken directly over the water travel time as follows:

$$
U(x, t)=e^{-\lambda t} \int_{0}^{\infty} J\left(t-K_{r} ; x_{0}\right) p_{w}\left(r ; x, x_{0}\right) d r
$$


where

$$
p_{w}\left(r ; x, x_{0}\right)=K p\left(K_{r} ; x, x_{0}\right)
$$

relates the water and solute travel time probability distributions.

Typical release consequence studies involve a distributed escape of contaminants from a repository. It is easy to demonstrate that such a situation causes no conceptual difficulties for the ensemble approach. Let $w(y)$ define a normalized release density and $f(t)$ define a rate function so that

$$
\int_{0}^{x_{0}} w(y) d y=1 \text { and } \quad \int_{0}^{\infty} f(t) d t=1
$$

The total release inventory is denoted by $W$, in curies. Then assuming concurrent release from each point, the release rate per unit of release region length is:

$$
J(t ; y)=W w(y) \bar{f}(t)
$$

The total distributed convective flux at an observation point $x$ beyond $x_{0}$ is

$$
U(x, t ; V)=e^{-\lambda t} W \int_{0}^{x_{0}} d y w(y) f(t-T(x ; y)) .
$$

now

$$
\bar{p}\left(r ; x, x_{0}\right)=\int_{0}^{x_{0}} d y w(y) p(r ; x, y)
$$


for $x>x_{0}$ defines a new p.d.f. for the distributed sources, and the expectation of Eq. 204 is given by

$$
\bar{U}(x, t)=e^{-\lambda t} w \int_{0}^{\infty} f(t-r) \bar{p}\left(r ; x, x_{0}\right) d \gamma,
$$

the observed flux at $x$ as measured over a boundary receiving all flow tube contents. Thus, the distributed source problem reduces to a point release with an average travel time p.d.f. (Eq. 205).

It seems implicit in the flow tube model of transport that the path lines must be parallel to the $x$-axis. However, this condition is actually not required, and the path lines (as curves in the $x-y$ plane) need only be laminar, allowing a one-to-one correspondence between the distance along each path and the $x$-axis. A family of non-intersecting curves with spacing determined by points of intersection on the $x$-axis must define the possible receiving boundaries over which the net observed outflow is measured. For example, the streamlines and potential contours of a gound water system determine an ideal flow tube model where measurements are taken along the contours. In such a manner the flow tube model represents transport from a localized release to an extended receiving boundary such as a segment of river. A similar viewpoint, but without an ensemble foundation, has been discussed (Ross and Koplik,1979). Non-uniformly Distributed Release:

An assumption of equal fractions of inventory being released into flow tubes defining the ensemble was so far an inherent aspect of the transport model. However, that assumption can be removed too. Indeed, suppose that $w[V]$ is the weight (curies) released without decay into all flow tubes having velocity $V(x)$. $w$ is a functional of $V$. Total release into the ensemble of tubes is:

$$
W \equiv\langle W[V]\rangle \quad \text {. }
$$


Stochastic convective flux becomes

$$
U(x, t ; V)=e^{-\lambda t} w[V] f\left(t-T\left(x ; x_{0}\right)\right)
$$

where $w V$ is viewed as a constant independent of $x$. Now by defining

$$
\bar{p}\left(r ; x, x_{0}\right)=\left\langle w[V] \delta+\left(r-T\left(x_{\dot{\theta}} x\right)\right)\right\rangle / W,
$$

which is a p.d.f. for $\gamma>0$, the expected flux is again given by Eq. 206. In particular, suppose that the velocity ensemble is a set of indexed velocities $V(x ; \alpha)$ for $\alpha$ in $\Omega$, and $\omega(\alpha)$ is a probability measure on $\Omega$. Then $\omega(A)$ represents the weight released into a set $A$ of tubes. The travel time p.d.f. is given by

$$
\bar{p}\left(\gamma ; x, x_{0}\right)=\int_{\Omega} \delta+\left(\gamma-T\left(x ; x_{0} ; \alpha\right)\right) d \omega(\alpha)
$$

for the expectation (Eq. 206). Thus, the non-uniformly distributed release $w[V]$ is equivalent to an ensemble with uniform release for an average travel time p.d.f. (Eq. 208).

An advantage of the ensemble approach is now apparent. The distributed source average (Eq. 205) can be applied to the non-uniform release p.d.f. (Eq. 208) to yield a compound travel time p.d.f. for an equivalent uniform point release. Such compounding of probability distributions for all varing aspects of a transport problem is a general property of the ensemble approach. Indeed, the possibility of stochastic boundary conditions can easily be included by similar methods.

Deterministic Simulations:

The explicit representation of the expectation (Eq. 200) suggests a method by which the outflow can be simulated by a deterministic system with a 
properly constructed release rate. The method requires finding a deterministic velocity $u(x)$ and a release rate function $F(t)$ such that the convective flux

$$
U(x, t)=F(t-\bar{T}(x))
$$

reproduces (Eq. 200) for $\lambda=0$ at some specifled $x$. If $u(x)$ can be taken as the ensemble average velocity, then only the function $F$ must be determined. For a fixed $x$, the release rate must satisfy

$$
F(t)=\bar{U}(x, t+\bar{T}(x)),
$$

but in general Eq. 210 will not determine the proper flux at other locations. Next consider conditions when Eq. 211 holds for all x. In order for Eq. 200 (with $\lambda=0$ and $x_{0}=0$ ) to have the form (Eq. 210) and satisfy the convection equation, the travel time p.d.f. must have the form

$$
p(r ; x)=f(r-\bar{T}(x))
$$

since Eq. 200 is a convolution. Then

$$
F(t)=\int_{0}^{\infty} J(\gamma) f(t-\gamma) d \gamma
$$

determines an equivalent deterministic flux by Eq. 210 for all $x$. Flux corrected for radioactive decay is obtained by multiplying Eq. 210 by the decay factor.

Observation of a Travel Time p.d.f.:

Some simple examples demonstrate that a travel time p.d.f. can be deduced directly from outflow observations, thus suggesting a possible experimental method. The idea is based on measuring cumulative solute outflow for a simple injection rate of tracer. Solute is injected at $x_{0}=0$ and observed at $x$, in the direction of flow. A subtle detail must first be considered in 
applying Eq. 200: $J(t)=W f(t)$ is the release rate into solution--not necessarily the injection rate. This distinction is due to the sorption process described by $K(x)$, and must be accounted for in an actual tracer experiment. To simplify equations, the solute is assumed non-decaying.

Example 1. An instantaneous point release is used, so that

$$
f(t)=\delta\left(t-t_{0}\right)
$$

Then flux (Eq. 200) gives

$$
U(x, t)=W p\left(t-t_{0} ; x\right)
$$

Observed cumulative outflow is proportional to the cumulative probability distribution:

$$
\int_{0}^{t} \bar{U}(x, \gamma) d \gamma=W \int_{0}^{t} p\left(\gamma-t_{0} ; x\right) d \gamma .
$$

Example 2. A constant release rate for a period $\Delta t=t_{2}-t_{1}$ is used, so that

$$
f(t)=\left\{\begin{array}{ccc}
1 / \Delta t & \text { if } t_{1}<t \leq t_{2} \\
0 & \text { otherwise }
\end{array} .\right.
$$

note that

$$
\frac{d f}{d t}=\frac{1}{\Delta t}\left[\delta\left(t-t_{1}\right)-\delta\left(t-t_{2}\right)\right]
$$


describes the release rate function in this case. Integeration of Eq. 200 by parts yields:

$$
\bar{U}(x, t)=W \int_{0}^{\infty} f^{\prime}(t-\gamma) P(\gamma ; x) d \gamma
$$

where

$$
P(t ; x)=\int_{0}^{t} p(r ; x) d \gamma
$$

And Eq. 216 gives

$$
U(x, t)=\frac{W}{\Delta t}\left[P\left(t-t_{1} ; x\right)-P\left(t-t_{2} ; x\right)\right] .
$$

Observe that Eq. 214 is obtained in the 1 imit $t_{1}, t_{2} \rightarrow t_{0}$. The cumulative outflow is given by

$$
\int_{0}^{t} \bar{U}(x ; \gamma) d=\frac{w}{\Delta t} \int_{t_{a}}^{t}[P(\gamma ; x)-P(\gamma-\Delta t ; x)] d \gamma
$$

for $t>t_{a}$ where $t_{a}$ denotes the first arrival time.

Example 3. An instantaneous release from a distributed sources occurs, but the travel time is known exactly. This case is described by example 1 with

$$
p\left(r ; x, x_{0}\right)=\delta\left(\gamma-T\left(x ; x_{0}\right)\right)
$$


for a single velocity. The flux for the distributed source is given by Eq. 204 as

$$
\bar{U}(x, t)=W \int_{0}^{x_{0}} d y_{\omega}(y) \delta\left(t-t_{0}-T(x ; y)\right),
$$

for $x>x_{0}$. Now using

$$
T(x)=T(x ; y)+T(y),
$$

Eq. 221 becomes

$$
\bar{U}(x, t)=W \int_{0}^{x_{0}} d y_{\omega}(y) \delta\left(T(x)-t+t_{0}-T(y)\right)
$$

which is, as expected, an initial value formulation of flux. Therefore

$$
\bar{U}(x, t)=W f\left[T^{-1}\left(T(x)-t+t_{0}\right)\right]
$$

where $f(y) \equiv V(y) w(y)$ defines the initial flux distribution. Here the inverse of $T(y)$ in $0 \leq y \leq x_{0}$ is assumed to exist.

A Compound Travel Time p.d.f.:

Long travel times will usually make it unreasonable for an experimenter to determine the transport behavior of a geologic region by a single tracer test. However, by a subdivision into adjacent smaller regions, simultaneous 
tracer experiments performed with in each subregion might make it feasible to estimate a compound travel time probability distribution. For example, suppose $p_{i}\left(\gamma_{;} x_{i}, x_{i-1}\right)$ were determined for the intervals $\left(x_{i-1}, x_{i}\right)$, $i=1, \ldots, n$ by an experiment such as example 1 . Further suppose that the expected flux is observable in each interval for any release at $x_{0}$. The outflow from $\left(x_{0}, x_{1}\right)$ determines the deterministic input flux for $\left(x_{1}, x_{2}\right)$, and so on over the entire region. Thus

$$
\begin{aligned}
J\left(x_{2}, t\right) & =\int_{0}^{\infty} \int_{0}^{\infty} J\left(t-r_{1}-r_{2}\right) p_{1}\left(r_{1}\right) p_{2}\left(r_{2}\right) d r_{1} d r_{2} \\
& =\int_{0}^{\infty} J(t-r) \bar{p}_{2}(r) d_{\gamma}
\end{aligned}
$$

where the compound p.d.f. is given by a convolution as

$$
\bar{p}_{2}(r)=\int_{0}^{\infty} p_{1}\left(r_{1}\right) p_{2}\left(r-r_{1}\right) d r_{1} .
$$

Then the flux at $x_{i}$ is determined simflarly to Eq. 224 from the sequence of convolutions given by

$$
\bar{p}_{j}(\gamma)=\int_{0}^{\infty} \bar{p}_{1-1}\left(\gamma_{1}\right) p_{j}\left(\gamma-r_{1}\right) d_{\gamma_{1}}
$$

with $p_{1}=p_{1}$. This compound p.d.f. (Eq. 226) describes the independent sum of travel times for the intervals $\left(x_{0}, x_{1}\right.$ through $\left(x_{i-1}, x_{j}\right)$. Notice also that the construction Eq. 224 and Eq. 225 is valid only without decay. Another equation results when decay is included.

Principles of Indeterminacy:

When applying the stochastic travel time method, certain cautions must be observed. They are related essentially to a simple conceptual difficulty: the "true" ensemble p.d.f. may be indeterminate because the influence of measurement processes is unknown. 
A first caution concerns the validity of Eq. 226. A p.d.f. constructed by the method Eq. 226 depends on an implicit assumption of the independence of velocity ensembles associated with each interval (subregion). In order that Eq. 226 be valid, the outflow from each interval must be uniformly distributed over the velocity ensemble of the next downstream interval. That is, a uniform mixing of solute over flow tubes needs to occur upon entering each subregion. This means that ensemble members of adjacent intervals must become uncorrelated over the length scales established by the subregions used to compound the p.d.f.

A further indetermininacy occurs because the travel time p.d.f. deduced from a tracer experiment depends on the injection distribution. For example, a particular solute injection method might cause an undetected non-uniform distribution of tracer over ensemble members, and then the estimated p.d.f. will describe only an equivalent uniform travel time distribution (Eq. 208). Such a p.d.f. used in the convolution (Eq. 226) could perturb the overall est imate.

Another indeterminancy is the dependence of an estimated p.d.f. for water travel time on the specific tracer employed, even after being corrected for retardation effects according to Eq. 202. Because the retardation factor is also a spatially stochastic quantity, experiments performed with tracers chemically different than an actual contaminant may yield non-representative results. The disparity occcurs because distinct chemical species may selectively sample different (effective) flow velocity ensembles. Moreover, this difficulty can be unavoidable for an extremely large retardation factor, since the experimental time frame may eliminate use of the actual contaminant as a tracer. In addition, the very concept of a retardation factor representation of chemical sorbtion processes may be questionable. Thatindeterminacy, however, is not within the scope of this ensemble discussion. (See Rao et al. 1979, DeSnedt and Wierenga 1979 for an entry into an extensive literature on that subject.)

A final caution concerns the fundamentals assumption of superposition of solute concentration pulses composing a general release pattern. In order to utilize the experiments suggested for a simple pulse or steps release, the 
travel time distribution determined must remain applicable for an arbitrary sum of such releases occurring over different times. Evidently that assumption requires the velocity ensemble to remain unaffected by solute concentration. Often that assumption is invoked by requiring solute to have only trace presents. However, such a dilute conditions requirement may not be within control; and moreover, the assumption itself may be invalid under some conditions. Perhaps, only direct experiments with the specific contaminant can decide this indeterminacy. In regards to this, it would be necessary to demonstrate that statistical moment generating functions are independent of the release function (see Eq. 215).

In conclusion, it is found that the same aspects which make the ensemble concept a flexible mathematical tool result in various indeterminacys that can not be removed without further information on the fundamental physical phenomena involved in transport processes. 


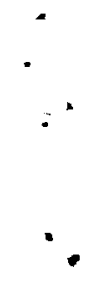

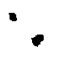

:

. 


\section{II . DEVELOPMENT OF DISPERSION EQUATIONS}

GENERAL RESULTS

The previous discussions have been concerned with direct calculation of an ensemble expected concentration or flux, and the evolution or transport equation for expected quantities was not emphasized. In this section, existence of a variety of dispersion equations for transport depending on particular initial or boundary conditions and flow velocity statistics is demonstrated.

The stochastic convective transport method is summarized by one equation:

$$
\langle c\rangle(x, t)=\iint s(y, \gamma)\langle G\rangle(x, t ; y, \gamma) d y d \gamma
$$

where $\langle G\rangle$ is the stochastic Green's function, which is an expectation of random Green's functions for the ensemble of convection equations given by

$$
L C \equiv \frac{\partial c}{\partial t}+V(x) \frac{\partial c}{\partial x}=s(x, t)
$$

That is, the integral operator defined by

$$
G s(x, t)=\iint s(y, \gamma) G(x, t ; y, \gamma) d y d \gamma
$$

is the inverse of $L$ defined in $E q .2: G=L-1$. Here $s(x, t)$ is a generalized source or sink function that describes deterministic initial or boundary conditions in the special cases by

$$
s(x, t)=f(x) \delta_{-}(t)
$$

and

$$
s(x, t)=\delta_{-}(x) f(t) \text {, }
$$


respectively. Random Green's functions must satisfy

$$
L G(x, t ; y, \gamma)=\delta(y-x) \delta(\gamma-t)
$$

for each velocity $V(x)$ in the ensemble. An initial value and boundary value formulation, Eq. 4 and 5 , are characterized by

$$
G(x, t ; y, \gamma)=\frac{1}{V(y)} \delta(t-\gamma-T(x ; y)) \theta(t-\gamma)
$$

(See Appendix C for details.)

A similar formulation for the stochastic convective flux $U$ is obtained as follows:

$$
L U \equiv \frac{1}{V(x)} \frac{\partial U}{\partial t}+\frac{\partial U}{\partial x}=s(x, t)
$$

with source given by Eq. 5. The Green's function for Eq. 8 is:

$$
G(x, t ; y, \gamma)=\theta(x-y) \delta(t-\gamma-T(x ; y))
$$

Expected flux is obtained by the same formula (Eq. 1).

A general transport equation is obtained by again inverting the integral operator defined in Eq. 1:

$$
\langle G\rangle^{-1}\langle C\rangle(x, t)=s(x, t)
$$

or

$$
\left\langle L^{-1}\right\rangle^{-1}\langle c\rangle(x, t)=s(x, t)
$$


Clearly, the expectation operation $\langle\bullet\rangle$ is required to yield some meaningful definition for the operator in Eq. 11. The operator given by

$$
\left\langle L^{-1}>-1-\langle L>\right.
$$

is then a generalized dispersion for the expected concentration (Eq. 1). Indeed, dispersive transport occurs when Eq. 12 is not a null operator, that is, if $\langle L-1\rangle-1 \neq\langle L\rangle$. Moreover, Ficklan transport occurs when Eq. 12 has the form

$$
-\frac{\partial}{\partial x}\left(D(x, t) \frac{\partial}{\partial x}\right)
$$

Unfortunately, the very generality of Eq. 11 renders it essentially useless as a tool for predicting transport because the detailed statistical representation of an ensemble expectation is not likely obtainable for a complicated groundwater system. What is needed is a method for constructing an approximation to Eq. 11 based entirely on only the statistical information obtainable from actual field measurements. A perturbation method by Keller (1964) fulfills the requirements.23

\section{A Perturbation Method}

Perturbation methods are based on the concept of obtaining corrections to solutions of Eq. 2 for small deviations of $V(x)$ from the mean velocity $u(x)$. The degree of smallness is described by the parameter $\varepsilon$ where

$$
V(x)=u(x)+\varepsilon W(x)
$$

with

$$
\langle w(x)\rangle=0 \quad \text {. }
$$


An $n$-th order corrected estimate of the expected value (Eq. 1) is given by

$$
\langle c\rangle(x, t)=c_{0}(x, t)+\sum_{k=1}^{n} \frac{e^{k}}{k !} \iint s(y, \gamma) G_{k}(x, t ; y, \gamma) \cdot d y d \gamma
$$

where

$$
G_{k}=\frac{\partial^{k}}{\partial \varepsilon^{k}}\left\langle G>\left.\right|_{\varepsilon=0}\right.
$$

and

$$
c_{0}(x, t)=\iint s(y, \gamma) G_{0}(x, t ; y, \gamma) d y d \gamma
$$

is the solution for mean velocity. The objective is to estimate the variations (Eq. 15) in the stochastic Green's function in terms of statistical moments of $w(x)$. Keller's method proceeds as follows. Let

$$
L=L_{0}+\varepsilon L_{1}
$$

where

$$
L_{0}=\frac{\partial}{\partial t}+u(x) \frac{\partial}{\partial x} \text { and } L_{1}=w(x) \frac{\partial}{\partial x} .
$$

$\mathrm{L}_{1}$ represents a stochastic perturbation. An approximate solution of Eq. 2 is then obtained by successive substitutions:

$$
\begin{aligned}
& c=L_{0}^{-1} S-\varepsilon L_{0}^{-1} L_{1} c \\
& c=L_{0}^{-1} S-\varepsilon\left(L_{0}^{-1} L_{1}\right) L_{0}^{-1} S+\varepsilon^{2}\left(L_{0}^{-1} L_{1}\right)^{2} c \\
& c=L_{0}^{-1} S-\varepsilon\left(L_{0}^{-1} L_{1}\right) L_{0}^{-1} S+\varepsilon^{2}\left(L_{0}^{-1} L_{1}\right)^{2} L_{0}^{-1} S-\varepsilon^{3}\left(L_{0}^{-1} L_{1}\right)^{3} c
\end{aligned}
$$


and so on to any order of $\varepsilon$.

Now using the properties

$$
\begin{array}{ll}
\left\langle\mathrm{L}_{0}^{-1} \mathrm{~g}\right\rangle=\mathrm{L}_{0}^{-1}\langle\mathrm{~g}\rangle & \text { (stochastic } \mathrm{g}) \\
\left\langle\mathrm{L}_{1} \mathrm{~g}\right\rangle=\left\langle\mathrm{L}_{1}\right\rangle \mathrm{g} & \text { (deterministic } \mathrm{g})
\end{array}
$$

and

$$
\left\langle L_{1}\right\rangle=0
$$

taking expectation of Eq. 19 gives

$$
\langle C\rangle=L_{0}^{-1} S+\varepsilon^{2} L_{0}^{-1}\left\langle L_{1} L_{0}^{-1} L_{1}>L_{0}^{-1} s+0\left(\varepsilon^{3}\right)\right.
$$

Resubstituting for $\mathrm{L}_{0}^{-1} \mathrm{~s}$ into Eq. 20 and rearranging terms yields Keller's equation:

$$
\left\{L_{0}-\varepsilon^{2}<L_{1} L_{0}^{-1} L_{1}>\left\langle C>=s+0\left(\varepsilon^{3}\right) .\right.\right.
$$

This method produces a transport equation correct for second order variations in velocity. Equation 21, however, actually represents a family of possible transport descriptions, depending on domains of the operators (Eq. 18) and the definition of expectation. Subsequent examples will demonstrate that behavior.

The expansion (Eq. 20) identifies the first two variations (Eq. 15) defined as integral operators. Since $c_{0}=L_{0} 1 \mathrm{~s}$, we have

$$
\begin{aligned}
& \mathrm{G}_{1} \equiv 0 \\
& \mathrm{G}_{2}=2 \mathrm{~L}_{0}^{-1}<\mathrm{L}_{1} \mathrm{~L}_{0}^{-1} \mathrm{~L}_{1}>\mathrm{L}_{0}^{-1} .
\end{aligned}
$$


And in general,

$$
G_{k}=(-1)^{k} k !<\left(L_{0}^{-1} L_{1}\right)^{k}>L_{0}^{-1}
$$

for $k=0,1,2, \ldots$. Evidently, the series (Eq. 14) converges to a solution if

$$
\varepsilon \quad\left\|<\left(L_{0}^{-1} L_{1}\right)^{k}>\right\|^{1 / k} \leq r<1
$$

for some definition of operator norm.

\section{Constant Mean Velocity Cases}

Evaluation of the expected operator triple product in Eq. 21 for constant mean velocity serves to exemplify most aspects of Keller's method. Thus suppose $\langle V(x)\rangle$ equals a constant $u$. The initial and boundary value formulations are considered as separate cases.

Initial conditions case:

The source term is Eq. 4 and the Green's function for $L_{0}$ is:

$$
G_{0}(x, t ; y, \gamma)=\theta(t-\gamma) \delta(x-y-u(t-\gamma))
$$

Evaluation of the triple product yields:

$$
\left\langle\mathrm{L}_{1} \mathrm{~L}_{0}^{-1} \mathrm{~L}_{1}>c=\int_{0}^{t}\left\{\left\langlew(x) w^{\prime}(y)>c_{x}(y, \gamma)+\left\langle w(x) w(y)>c_{x x}(y, \gamma)\right\} d \gamma\right.\right.\right.
$$

with $y=x-u(t-r)$.

The domain of Eq. 25 is restricted to functions such that $c(x, t)=0$ if $t<0$. Spatial velocity correlation is defined by

$$
R(x, y)=\langle w(x) w(y)\rangle
$$


and satisfies a symmetry condition:

$$
R(x, y)=R(y, x)
$$

In deriving Eq. 25, it is necessary to decide on the order of performing the expectation and differentiation operations on the product $w(x) w(y)$. Evidently the velocity ensemble requires an additional independent condition for evaluation of $\left\langle w(x) w^{\prime}(y)\right\rangle$. A natural condition might be imposed by requiring

$$
<w(x) w^{\prime}(y)>=\frac{\partial R}{\partial y}(x, y) \quad \text {. }
$$

However, such a requirement is not in general necessary. A particular ensemble could have the same correlation (Eq. 26) yet have a vanishing correlation between velocity and its derivative, that is

$$
\left\langle w(x) w^{\prime}(y)>\equiv 0\right.
$$

Both situations have distinct physical consequences for solute transport.

If the velocity variation is stationary, then an autocorrelation function $A(x)$ exists so that

$$
R(x, y)=\mu^{2} A(x-y)
$$

where $\mu^{2}$ denotes the variance in velocity. In that case the dispersion operator (Eq. 25) becomes

$$
<L_{1} L_{0}^{-1} L_{1}>c=\frac{\mu^{2}}{u} \int_{0}^{u t}\left\{-A^{\prime}(y) c_{x}(x-y, t-y / u)+A(y) c_{x x}(x-y, t-y / u)\right\} d y .
$$

The dispersive transport equation for each case is:

$$
\frac{\partial}{\partial t}\langle c\rangle+u \frac{\partial}{\partial x}\langle c\rangle=\left\langle L_{1} L_{0}^{-1} L_{1}\right\rangle\langle c\rangle
$$

for $\mathrm{t}>0$ and $\varepsilon=1$. 
Two extreme ensembles are of particular interest. Suppose that the correlation (Eq. 26) equals a constant variance $\mu^{2}$ and (Eq. 27) holds, then the dispersion term of Eq. 31 is:

$$
\left\langle L_{1} L_{0}^{-1} L_{1}\right\rangle\langle C\rangle=\mu^{2} \int_{0}^{t} \frac{\partial^{2}}{\partial x^{2}}\langle C\rangle(x-u(t-\gamma), \gamma) d \gamma .
$$

For the other extreme, suppose that

$$
R(x, y)=\mu^{2} \delta(x-y) \quad .
$$

The condition (Eq. 27) when used in Eq. 25 results in an undefined (unbound) operator, however. Therefore, the condition (Eq. 28) is imposed instead. Together, Eq. 33 and 28 result in Fickian dispersion given by

$$
\left\langle L_{1} L_{0}^{-1} L_{1}\right\rangle\langle c\rangle=\frac{\mu^{2}}{2 u} \frac{\partial^{2}}{\partial x^{2}}\langle c\rangle
$$

Notice that the integral

$$
\frac{\mu^{2}}{u} \int_{0}^{u t} \delta(y-u t) d y=\frac{\mu^{2}}{2 u}
$$

used to obtain Eq. 34 has units of a dispersion coefficient, (length $2 / t$ ime).

The general transport equation (Eq. 31) appears to allow the possibility of a greater variety of solutions different from that determined in the original expansion Eq. 14 or Eq. 20. Since Eq. 31 usually results in a second order partial differential equation, whereas the original stochastic convection equation is first order, additional conditions would seem to be required in order to establish a unique expected concentration. This difficulty requires restricting the dispersion operator's domain to 
concentration functions allowed by Eq. 2. For example, consider the transport described by Eq. 32. The exact solution for the original problem is

$$
\langle c\rangle(x, t)=\sum_{k=0}^{\infty} f^{(k)}(x-u t) \frac{(-\varepsilon t)^{k}}{k !}\left\langle w^{k}\right\rangle
$$

for $t \geq 0$. Direct calculation of the terms in Eq. 20 leads to results consistant with the expansion (Eq. 35):

$$
\begin{aligned}
c_{0}(x, t) & =L_{0}^{-1} s=f(x-u t) \\
L_{0}^{-1}\left\langle L_{1} L_{0}^{-1}\right\rangle c_{0} & =\frac{\mu^{2}}{2} t^{2} f^{(2)}(x-u t) .
\end{aligned}
$$

The second order expansion (Eq. 20), however, can be shown to satisfy the transport equation given by Eq. 31 with

$$
\left\langle L_{1} L_{0}^{-1} L_{1}\right\rangle\langle C\rangle=t_{\mu}{ }^{2} \frac{\partial^{2}}{\partial x^{2}}\langle C\rangle+0\left(\varepsilon^{4}\right) .
$$

The Fickian dispersion ( $E q .36$ ) amounts to assuming a restricted domain for Eq. 32 with functions of form (Eq. 16). Notice that Eq. 36 is associated with a Gaussian constant velocity ensemble, and determines the exact transport equation.

Boundary Conditions Case:

The stochastic transport is now described by

$$
L_{0} C=\frac{1}{u} \frac{\partial C}{\partial t}+\frac{\partial C}{\partial x}, L_{1} C=\frac{w}{u}(x) \frac{\partial C}{\partial x},
$$

and

$$
s(x, t)=\delta(x) f(t) / u \text {. }
$$


The Green's function for a boundary condition formulation is:

$$
G_{0}(x, t ; y, \gamma)=\theta(x-y) \delta_{-}(t-\gamma-(x-y) / u)
$$

and it corresponds to solutions given by

$$
c_{0}(x, t)=\theta(x) f(t-x / u) / u
$$

for $t-x / u \geq 0$. Evaluation of the dispersion operator (divided by $u$ ) yields

$$
\left\langle L_{1} L_{0}^{-1} L_{1}\right\rangle c=\frac{R(x, x)}{u^{2}} \frac{\partial C}{\partial x}-\int_{0}^{x} \int^{\frac{R(x, y)}{2}} \frac{\partial C}{\partial y}(y, \gamma) \cdot \frac{\partial \delta}{\partial y}(t-\gamma-(x-y) / u) d \gamma d y
$$

or using integration by parts gives

$$
\begin{aligned}
\left\langle L_{1} L_{0}^{-1} L_{1}>c\right. & =\frac{R}{u^{2}}(0, x) c_{x}(0, t-x / u) \\
& +\frac{1}{u^{2}} \int_{0}^{x}\left\{R_{y}(x, y) c_{x}(y, \lambda)+R(x, y) c_{x x}(y, \lambda)\right\} d y
\end{aligned}
$$

with $\lambda=t-(x-y) / u$. A constant correlation equal to $\mu^{2}$ used in Eq. 42 yields

$$
\left\langle L_{1} L_{0}^{-1} L_{1}\right\rangle\langle C\rangle=\frac{\mu^{2}}{u^{2}}\left[\langle C\rangle_{x}(0, t-x / u)+\int_{0}^{x}\langle C\rangle_{x x}(y, \lambda) d y\right] .
$$

The delta correlation (Eq. 33) and the condition (Eq. 28) again yield Fickian dispersion given by

$$
\left\langle L_{1} L_{0}^{-1} L_{1}\right\rangle\langle C\rangle=\frac{\mu^{2}}{2 u^{2}} \frac{\partial^{2}}{\partial x^{2}}\langle C\rangle \quad(x>0)
$$


Notice that in these cases the transport equation (Eq. 31) has dispersion operator equal to Eq. 41 multiplied by $u$.

Variance Estimation

Keller's perturbation method provides a simple formula for estimating variance in concentration distributions. The variance defined by

$$
\operatorname{Var}[c] \equiv\left\langle(c-\langle c\rangle)^{2}\right\rangle
$$

depends on position $x$ and time $t$ and represents the deviation of concentration ensemble members from the expected value. Squaring the difference between Equationss 19 and 20 and taking the expectation as in Eq. 45 yields a second order estimate of variance:

$$
\left.\operatorname{Var}[c]=\varepsilon^{2}<\left(L_{0}^{-1} L_{1} C_{0}\right)^{2}\right\rangle+0\left(\varepsilon^{3}\right)
$$

with $c_{0}=L_{0}^{-1} s$. Using the expectation (Eq. 20) as an estimate of $c_{0}$ in Eq. 46 gives

$$
\operatorname{Var}[C]=\varepsilon^{2}\left\langle\left(L_{0}^{-1} L_{1}\langle C\rangle\right)^{2}\right\rangle+0\left(\varepsilon^{3}\right)
$$

Apparently, the operator $\mathrm{eL}_{0}^{-1} \mathrm{~L}_{1}$ propagates deviations from the expectation for each velocity ensemble member.

A few simple examples serve to demonstrate Eq. 47 . Consider first the constant velocity and correlation case (Eq. 35) with deterministic initial conditions. We have

$$
L_{0}^{-1} L_{1}\langle c\rangle=w \int_{0}^{t}\langle c\rangle_{x}(x-u(t-\gamma), \gamma) d \gamma,
$$


and

$$
L_{0}^{-1} L_{1} C_{0}=w t \quad f^{(1)}(x-u t)
$$

gives

$$
\operatorname{Var}[c]=\left(t f^{(1)}(x-u t)\right)^{2} \operatorname{Var}[v]
$$

The expansion (Eq. 19) gives another indirect method of calculating Eq. 46 based on

$$
G_{1} S=-\left(L_{0}^{-1} L_{1}\right) L_{0}^{-1} S
$$

with

$$
G_{1}=\left.\frac{\partial G}{\partial \varepsilon}\right|_{\varepsilon=0}
$$

defining an operator. Putting this into Eq. 46 gives

$$
\operatorname{Var}[c]=\left\langle\left(\varepsilon G_{1} s\right)^{2}\right\rangle
$$

In view of Eq. 35, Eq. 50 again yields Eq. 48. The more general situation with constant $u$ but any autocorrelation follows. We have

$$
L_{0}^{-1} L_{1}\langle c\rangle=\int_{0}^{u t} w(x-u t+y)\langle c\rangle_{x}(x-u t+y, y / u) d y / u
$$


and

$$
L_{0}^{-1} L_{1} c_{0}=\frac{f}{u}^{(1)}(x-u t) \int_{0}^{u t} w(x-u t+y) d y
$$

making use of Taylor's theorem gives

$$
\left\langle\left(\int_{0}^{z} w(x-z+y) d y\right)^{2}\right\rangle=2 \mu^{2} \int_{0}^{z} d y \int_{0}^{y} A\left(y^{\prime}\right) d y^{\prime}
$$

where $z=u t$, which when applied in Eq. 46 yields:

$$
\operatorname{Var}[c]=\left(\frac{\varepsilon \mu}{u} f^{(1)}(x-u t)\right)^{2} 2 \int_{0}^{u t} d y \int_{0}^{y} A\left(y^{\prime}\right) d y^{\prime} .
$$

A delta correlation (Eq. 33) gives the special result

$$
\operatorname{Var}[c]=\frac{t}{u}\left(f^{(1)}(x-u t)\right)^{2} \operatorname{Var}[V]
$$

by letting $\operatorname{Var}[V]=(\varepsilon \mu)^{2}$. Notice that $E q .48$ is directly proportional to $t^{2}$, whereas Eq. 52 increases 1 inearly. Moreover, Eq. 52 is reduced by increasing $u$. Provided that the derivative of an initial concentration vanishes, both special cases show that the variance vanishes after sufficiently large times, corresponding to the passage of solute observed at $x$.

In actual numerical applications which produce expected concentration as a solution of Keller's equation expressed as a system of finite difference equations, the variance estimate (Eq. 47) is most appropriate. Oster et. al. (1980) and Gibbs (1980) have discussed mathematical details for that approach. Since it may be difficult to identify terms of various $\varepsilon$ orders in 
a solution of $\mathrm{Eq} .21$ it seems important to distinguish the differences between such a solution and the actual expected concentration, which is given by

$$
\langle C\rangle=\sum_{k=0}^{\infty}(-\varepsilon)^{k}<\left(L_{0}^{-1} L_{1}\right)^{k}>c_{0} .
$$

Applying the same perturbation expansion to Eq. 21, however, yields solutions

$$
\langle c\rangle=\sum_{k=0}^{\infty} e^{2 k}\left(\left\langle\left(L_{0}^{-1} L_{1}\right)^{2}>\right)^{k} c_{0} .\right.
$$

As required Equations 53 and 54 are identical through second order terms, but appear to differ on higher order terms, as expected. This disparity may yield different estimates of Equations 46 and 47 , depending on the behavior of the operator $L_{0}^{-1} L_{1}$.

\section{Stochastic Convective Flux}

A stochastic transport problem of particular relevancy to consequence predictions is described by Eq. 2 with

$$
s(x, t)=V(x) \delta_{-}(x) f(t)
$$

for a boundary value formulation given by Eq. 7. This represents stochastic transport described by concentrations given by

$$
c(x, t)=\theta(x) f(t-T(x)),
$$

which have a deterministic boundary value specified. By dividing out velocity in Eq. 2, the problem is exactly the same as Eq. 8 with source (Eq. 5) (assuming $V(x)>0$ of course). The source (Eq. 55) represents a stochastic input flux, whereas in contrast Eq. 5 represents the deterministic case. This latter case can be solved by defining Equations 37 and 38 with mean velocity 
depending on $x$. Evaluation of the dispersion operator again yields Eq. 42, but with $\bar{T}(x ; y)$ replacing $(x-y) / u$ in this more general situation. To solve the problem for Eq. 55 define

$$
L_{0}=\frac{1}{u(x)} \frac{\partial}{\partial t}+\frac{\partial}{\partial x} \text { and } L_{1}=w_{1}(x) \frac{\partial}{\partial t}
$$

where

$$
1 / u(x)=\langle 1 / V(x)\rangle \text { and } w_{1}=1 / V-1 / u \text {. }
$$

Now a second order estimate of expected concentration was prevfously obtained by using stochastic travel time and is given by Eq. 4.31. The method amounted to expanding Eq. 56 in $\varepsilon$ for velocity given as Eq. 13, or using Eq. 14. Applying Eq. 50 the variance estimate is:

$$
\operatorname{Var}[c]=\left(e^{(1)}(t-T(x))^{2} \int_{0}^{x} \int_{0}^{x} \frac{R(y, z)}{u^{2}(y) u^{2}(z)} d y d z .\right.
$$

Equation 46 gives

$$
\operatorname{Var}[c]=\left(c^{(1)}(t-\mathcal{T}(x))^{2} \int_{0}^{x} \int_{0}^{x} R_{1}(y, z) d y d z\right.
$$

with correlation $R_{1}$ defined by Eq. 26 for $w_{1}$. The first order expansion

$$
\frac{1}{v}-\frac{1}{u}=-\varepsilon \frac{w}{u^{2}}=\varepsilon w_{1}
$$

reduces Eq. 59 to Eq. 58, however. For fluctuations about a harmonic mean u described by Eq. 57 the dispersive transport equation is given by

$$
\frac{\partial}{\partial t}\langle c\rangle+u(x) \frac{\partial}{\partial x}\langle c\rangle=\varepsilon^{2} u(x)\left\langle L_{1} L_{0}^{-1} L_{1}\right\rangle\langle c\rangle+\bar{s}
$$


where

$$
\bar{s}(x, t)=u(x) \delta(x) f(t),
$$

and the dispersion operator (divided by $u$ ) is

$$
\left\langle L_{1} L_{0}^{-1} L_{1}>c=\frac{\partial^{2}}{\partial t^{2}} \int_{0}^{x} R_{1}(x, y) c(y, t-\bar{T}(x ; y)) d y .\right.
$$

If the correlation function $R_{1}$ is given by Eq. 33, then Eq. 61 becomes

$$
<L_{1} L_{0}^{-1} L_{1}>c=\frac{\mu^{2} \partial^{2} c}{2} \frac{\partial t^{2}}{.}
$$

On the other hand, if the first order approximation

$$
R_{1}(x, y) \doteq \frac{R(x, y)}{u^{2}(x) u^{2}(y)}
$$

holds and $\mathrm{R}$ is given by Eq. 33 instead, then Eq. 61 becomes

$$
\left\langle L_{1} L_{0}^{-1} L_{1}>c=\frac{\mu^{2}}{2 u^{4}(x)} \frac{\partial^{2} c}{\partial t^{2}} .\right.
$$

Transport equations defined by Equations 62 and 63 correspond exactly to the case of purely random, stationary, Gaussian processes previously discussed. The second order solution (Eq. 20) is determined by

$$
c_{0}(x, t)=f(t-\bar{T}(x))
$$


and

$$
L_{0}^{-1}<L_{1} L_{0}^{-1} L_{1}>c_{0}=f^{(2)}(t-\bar{T}(x)) \frac{1}{2} \int_{0}^{x} \int_{0}^{x} R_{1}(y, z) d y d z
$$

for $x \geq 0$. Notice that this result differs from that for second order fluctuations about an arithmatic mean velocity, which gave Eq. 4.31. In particular, second order fluctuation about the mean velocity is described by

$$
L_{1} C=\left(\frac{-w(x)}{u^{2}(x)}+\varepsilon \frac{w^{2}(x)}{u^{3}(x)}\right) \frac{\partial C}{\partial t}
$$

for Eq. 13, and the expectation of Eq. 64 does not vanish as required in the derivation of Keller's equation (Eq. 21). By retaining the first order term in the expectation of Eq. 19, the expansion becomes

$$
\langle C\rangle=C_{0}-\varepsilon L_{0}^{-1}\left\langle L_{1}\right\rangle C_{0}+\varepsilon^{2} L_{0}^{-1}\left\langle L_{1} L_{0}^{-1} L_{1}>C_{0}+0\left(\varepsilon^{3}\right) .\right.
$$

Thus an additional second order term from Eq. 64 will appear in Eq. 65. Evaluating the terms in Eq. 65 gives

$$
L_{0}^{-1}\left\langle L_{1}\right\rangle c_{0}=\varepsilon \int_{0}^{x} \frac{\left\langle w^{2}(y)\right\rangle}{u^{3}(y)} d y f^{(1)}(t-\bar{T}(x))
$$

and

$$
L_{0}^{-1}<L_{1} L_{0}^{-1} L_{1}>c_{0}=\frac{1}{2} \int_{0}^{x} \int_{0}^{x} \frac{R(y, z)}{u^{2}(y) u^{2}(z)} d y d z f^{(2)}(t-\bar{T}(x))+0(\varepsilon) \text {. }
$$


Terms of order $\varepsilon$ in Eq. 67 then contribute to third order terms in Eq. 65 and are neglected. Indeed, Keller (1964) has shown that the expectation satisfies

$$
\left\{L_{0}+\varepsilon^{2}\left(\left\langle L_{2}\right\rangle-\left\langle L_{1} L_{0}^{-1} L_{1}\right\rangle\right)\right\}\langle C\rangle=s
$$

for a stochastic equation given by

$$
\left(L_{0}+\varepsilon L_{1}+\varepsilon^{2} L_{2}\right) c=s
$$

with $\left\langle L_{1}\right\rangle=0$. For this application (Eq. 57), Eq. 64 with $\varepsilon=0$ and

$$
L_{2}=\frac{w^{2}(x)}{u^{3}(x)} \frac{\partial}{\partial t}
$$

define the operators, while the source is Eq. 5.

An alternative approach to solving the stochastic transport problem for Eq. 8 with Eq. 5 is to use Eq. 2 directly with Eq. 55 as a stochastic source. The problem Decomes

$$
\left(L_{0}+\varepsilon L_{1}\right) C=S+E S_{1}
$$

with

$$
L_{0}=\frac{1}{u(x)} \frac{\partial}{\partial t}+\frac{\partial}{\partial x} \text { and } L_{1}=\frac{w(x)}{u(x)} \frac{\partial}{\partial x} \text {, }
$$

for a stochastic source

$$
s_{1}(x, t)=\frac{w(x)}{u(x)} \delta_{-}(x) f(t) .
$$


Gibbs (1980) has shown that the expected solution to Eq. 71 is then

$$
\langle c\rangle=c_{0}+\varepsilon^{2} L_{0}^{-1}\left\langle L_{1} L_{0}^{-1} L_{1}\right\rangle c_{0}-\varepsilon^{2} L_{0}^{-1}\left\langle L_{1} L_{0}^{-1} s_{1}\right\rangle+0\left(\varepsilon^{3}\right)
$$

and Keller's equation becomes

$$
\left\{L_{0}-\varepsilon^{2}\left\langle L_{1} L_{0}^{-1} L_{1}\right\rangle\right\}\langle C\rangle=s-\varepsilon^{2}\left\langle L_{1} L_{0}^{-1} s_{1}\right\rangle+O\left(\varepsilon^{3}\right)
$$

assuming

$$
\left\langle L_{1}\right\rangle=\left\langle s_{1}\right\rangle=0
$$

An important aspect of stochastic convective transport is the multitude of disguised representations possible for the dispersion operator. In particular, by applying repeated integration by parts and converting time derivatives to spatial derivatives, Eq. 61 becomes

$$
\begin{aligned}
\left\langle L_{1} L_{0}^{-1} L_{1}\right\rangle c & =\frac{\left\langle w_{0}^{2}\right\rangle}{u} \frac{\partial c}{\partial t}-\left\langle w_{0} \frac{\partial}{\partial x} w_{0}\right\rangle c \\
& +\frac{1}{u} \iint_{-\infty}^{x} \delta(t-\gamma-T(x ; y)) D_{y}(x) c(y, \gamma) d y d \gamma
\end{aligned}
$$

where

$$
D_{y}(x) c=<\frac{\partial}{\partial y}\left(u(y) w_{0}(x) \frac{\partial}{\partial y} w_{0}(y)\right)>c
$$

and

$$
w_{0}(x) \equiv w_{1}(x) u(x)=\frac{u(x)}{V(x)}-1 .
$$


The variation $w_{1}$ is relative to a harmonic mean velocity. For a second order variation about the arithmatic mean, the transport is described by Eq. 68 with Eq. 70, and the dispersion operator (divided by $u$ ) is given by

$$
\left\langle L_{1} L_{0}^{-1} L_{1}\right\rangle-\left\langle L_{2}\right\rangle
$$

for Eq. 76 with

$$
w_{0}(x)=-w(x) / u(x)
$$

Then Eq. 79 equals Eq. 76 with the first term removed. In either case, it is apparent that the dispersion operator can assume many different forms that depend on the explicit character of the velocity ensemble as reflected by expectations, $\left\langle w_{0}(x) w_{0}(n)(y)\right\rangle(n=0,1,2)$, involving both the variation and its derivatives. Now because the source function was arbitrary, the possible transport equations based on Eq. 76 have a generality exceeding the present problem defined by Eq. 55. Indeed, the dispersion operators can have domains restricted to all $c_{0}$ for sources (Eq. 55) without altering second order approximate solutions for mean concentration. That is, a restricted dispersion operator is determined by

$$
\begin{aligned}
\left\langle L_{1} L_{0}^{-1} L_{1}>c_{0}\right. & =L_{0}\left\{\frac{1}{2} \int_{0}^{x} \int_{0}^{x} R_{1}(y, z) d y d z f^{(2)}(t-\bar{T}(x))\right\} \\
& =\int_{0}^{x} R_{1}(x, y) d y f^{(2)}(t-\bar{T}(x)) \\
& =\int_{0}^{x} R_{1}(x, y) d y u(x) \frac{\partial}{\partial x}\left(u(x) \frac{\partial}{\partial x}\right) c_{0} .
\end{aligned}
$$


and the transport satisfies Eq. 60 with

$$
\left.u(x)<L_{1} L_{0}^{-1} L_{1}\right\rangle=\int_{0}^{x} R_{1}(x, y) d y u^{2} \frac{\partial}{\partial x}\left(u \frac{\partial}{\partial x}\right)
$$

determining the dispersion operator. Similarly, by using Eq. 68 with Equations 66 and 67 the restricted dispersion operator is given by

$$
\begin{aligned}
u\left\{\left\langle L_{1} L_{0}^{-1} L_{1}\right\rangle-\left\langle L_{2}\right\rangle\right\} & =-u(x) \int_{0}^{x} \frac{\partial R_{0}}{\partial x}(x, y) d y \frac{\partial}{\partial x} \\
& +\frac{\partial}{\partial x}\left(u(x) \int_{0}^{x} R_{0}(x, y) d y \frac{\partial}{\partial x}\right)
\end{aligned}
$$

where

$$
R_{0}(x, y)=R(x, y) / u^{2}(y) \text {. }
$$

Transport is still given by Eq. 60 but with Eq. 82 replacing Eq. 81 in this second order velocity variation case. As a consequence of the restricted domains, Equations 81 and 82 describe Fickian convective-dispersive transport with an altered convective velocity term. That is, for example, the first order derivative term in Eq. 82 adds to mean velocity in the transport equation (Eq. 60). The effect of this term depends on the particular statistical interpretation of $R_{x}(x, y)$ as previously mentioned. It must be emphasized that these results are based on the special source (Eq. 55) and a second order approximation of expected concentration. An interesting aspect of Eq. 82 is that it suggests the well known experimental observation of a dispersion coefficient directly proportional to local flow velocity. But the derivation indicates that such behavior depends on special boundary conditions. Indeed, the failure of the classical convective-dispersion equation to properly predict transport under certain conditions seems clarified by the changing character of the dispersion operator when restricted to different domains. 
-

.

3 


\section{CONCLUSIONS AND RECOMMENDATIONS}

The classical convective-dispersion equation as a theory of mass transport fails at the megascopic scale of a geologic system as a consequence of uncertain flow velocity variations occurring at the macroscopic scale. Uncertainty in the velocity is an unavoidable aspect associated mainly with the inherent spatial variability of hydraulic conductivity in a hydrologic system. Measurement accuracy and sample volume size (scale) fundamentally determine the magnitude of uncertainty, as represented by a statistical distribution of velocity. As a result, modeling of solute transport in a groundwater system as a diffusion process based on laboratory or field determined dispersivity coefficients will prove unreliable. Attempts to retain a diffusion description by introducing effective dispersivity coefficients are generally not successful because such coefficients may only describe dispersion within the specific region and period of their estimation.

The overall consequence of not accounting for uncertainty in some situations appears to be a serious under estimation of escaping contaminant mass. In particular, if the inherent velocity variation associated with an actual megascopic dispersion has a relative preponderance of high velocity paths so that the travel time probability is skewed, then the arrival time of contaminant as measured at some detection level may be less than that predicted by a mean velocity and laboratory determined dispersion coefficient alone. Indeed, if the travel time's variance is large relative to the mean then the detailed nature of its distribution will invariably be important in predicting contaminant outflow. Since the travel time variance reflects the overall effect of the velocity's spatial covariance, it therefore is a fundamentally necessary statistical property of a proper transport theory.

A stochastic convective flow method is found to constitute a more fundamental approach for characterizing dispersive mass transport in a hydrologic system. The method constitutes an extension, since the classical Fickian diffusion viewpoint is included as a special case. The approach requires averaging of the convective flow equation over an ensemble of realizable velocity fields representing possible flow states of a system. It 
is thus indicated that a statistical description of hydraulic conductivity, porosity, and potential contours is essential. Transport is described by expected values of concentration obtained with a particular averaging method. In addition, the approach is general enough to encompass problems of estimating prediction uncertainity associated with measurement errors in hydrologic properties. Thus an ensemble may represent either (1) the multitude of component velocity paths traveled by solute comprising the expected transport, or (2) the variety of possible descriptions associated with measurement uncertainty. Individual ensemble numbers (realizations) in the first case may not necessarily be actually observable, whereas the expected transport in the second case may not be observalbe. That is, the expectation in case (2) need not represent a realizable result, and may have only statistical significance.

An unavoidable difficulty imposed by the stochastic nature of transport is the result that a single governing partial differential equation no longer describes solute movement under all situations. Instead, a number of computational algorithms may be required for different conditions. In particular, if velocity variation or uncertainty is small relative to its mean, then perturbation methods utilizing only the mean and spatial covariance are applicable. On the other hand, extreme variation may demand application of an idealized random motion model utilizing an unestablished level of statistical information. Such a model would require extension of random walk methods. In certain circumstances, a direct averaging approach using Monte Carlo methods may be called for.

The major conclusion is that present computer models of transport in a groundwater system will require considerable revision in order to provide realistic release consequence predictions. Furthermore, there is no substitute for extensive site specific field measurements, when attempting a serious transport modeling effort, and the statistical behavior cannot be neglected. 


\section{REFERENCES}

1. Bear, J. 1972. Dynamics of Fluids in Porous Media. New York, Elsevier.

2. Puckens, J. F., J. A. Cherry, N. W. Gillham, and W. F. Merrit. 1978. "Field Studies of Dispersion in a Shallow Sandy Aquifer." In Invitational Wel1-Testing Sympostum. Proceedings October 19-21, 1977, Berkeley, Calffornta. LBL-7027.

3. Frled, J. J. 1975. Ground Water Pollution. Elsevier, New York.

4. Biggar, J. W., and D. R. Nielsen. 1976. "Spatial Variability of the Leaching Characteristics of a Field Soil." Water Resources Res. $12(1): 78-84$.

5. Simpson, E. S., ed. 1979. "Progress and Problems in the Analysis and Prediction of Sub-Surface Mass Transport." In A Topical Report to the Nuclear Regulatory Commission. NRC-04-78-275. Dept. of Hydrology and Water Resources, Univ. of Arizona, Tucson, Arizona.

6. Sposito, G., V. K. Gupta, and R. N. Bhattacharya. 1979. "Foundation Theories of Solute Transport in Porous Media: A Critical Review." Advances in Water Resources 2:59-68.

7. Naff, R. L. 1978. "A Continuum Approach to the Study and Determination of Field Longitudinal Disperson Coefficients." Ph.D. dissertation. New Mexico Institute of Mining and Technology, Socouro, New Mexico. $176 \mathrm{pp}$.

8. Gelhar, Q. W., A. L. Gutjaher, and R. L. Naff. 1979. "Stochastic Analysis of Macrodisperson in a Stratified Aquifer." Water Resources Res. 15(6):1387-1397.

9. Tang, D. H., and G. F. Pinder. 1979. "Analysis of Mass Transport with Uncertain Physical Parameters." Water Resources Res. $15(5): 1147-1155$.

10. Delhomne, J. P. 1979. "Spatial Varfability and Uncertainty in Groundwater Flow Parameters: A Geostatistical Approach." Water Resources Res. 15(2): 269-280.

11. Gibbs, J. W. 1902. Elementary Principles in Statistical Mechanics. Dover Pub. Inc., New York, C. 1960.

12. Scheidegger, A. E. 1954. "Statistical Hydrodynamics in Porous Media." J. Applied Physics 25(8)994-1001.

13. Oster, C. A., A. G. Gibbs, and D. H. Tang. 1979. Analysis of a Numerical Solution to the One-Dimensional Stochastic Convection Equation. PNL-SA-8059, Pacific Northwest Laboratory, Richland, Washington 99352. Submitted to J. Advance in Water Resources. 
14. Adomian, G. 1970. "Random Operation Equations in Mathematical Physics. I. J. Math. Phys. 11(3):1069-1084.

15. Carslaw, H. S., and J. C. Jaegar. 1963. Operational Methods in Applied Mathematics, Dover, New York.

16. Gel'Fand, I. M., and A. M. Yaglom. 1960. "Intergration in Functional Space and its Applications in Quantum Physics." J. Math. Phys. $1(1): 48-69$.

17. Fox, R. F. 1972. "Contributions to the Thoery of Stochastic Processes. J. Math. Phys. 13:1196-1207.

18. Wax, N. 1954. Selected Papers on No1se and Stochastic Processes. Dover Pub., New York.

19. Riesiz, F., and B. Sy.-Nagy. 1965. Functional Analysis. Frederich Ungar Pub., New York.

20. Ross, B., and C. M. Kopl 1k. 1979. "A New Numerical Method for Solving the Solute Transport Equation." Water Resources Res. 15(4):949-955.

21. Rao, P. S. C., J. M. Davidson, R. E. Jessup, and H. M. Selim. 1979. "Evaluation of Conceptual Modals for Describing Nonequilibrium Adsorption-Desorption of Pesticides During Steady-Flow in Solls." Soll Sci. Soc. Am. J. 43:22-28.

22. DeSnedt, F., and P. J. Wierenga. 1979. "A Generalized Solution for Solute Flow in Soils with Mobile and Immobile Water." Water Resources Res. 15(5):1137-1141.

23. Keller, J. B. 1964. "Stochastic Equations and Wave Propagation in Random Media" in Stochastic Processes in Mathematical Physics and Engineering. Proceedings of Symp. In Appl. Math. 16, Amer. Math. Soc. Providence, pp. $145-170$.

24. Gibbs, A. G. 1980. A Perturbation Method for Stochastic Operator Difference Equations. PNL-SA-8409. To be submitted for publication.

25. Smith, L. and F. W. Schwartz. 1980. Mass Transport I. A Stochastic Analysis of Macroscopic Dispersion. Water Res. Research. $16(2): 303-313$.

26. Matheron, G. and G. DeMarsily. 1980. Is Transport in Porous Media Always Diffusive? A Counterexample. Water Res. Research. 16(5):901-917. 


\section{APPENDIX A}

TWO EXAMPLES OF A GAUSSIAN CONSTANT VELOCITY ENSEMBLE

FOR AN INITIAL VALUE FORMULATION

The velocity P.d.f. is assumed independent of $x$. An effective dispersion function $D(x, t)$ required to be independent of initial concentration values satisfies

$$
\frac{\partial G}{\partial t}+u(t) \frac{\partial G}{\partial x}=\frac{\partial}{\partial x}\left(D(x, t) \frac{\partial G}{\partial x}\right)
$$

in $-\infty<x<\infty$ for $u(t)=\bar{V}(t)$ where

$$
G(x, t ; y)=P \star(x-y ; t)=P((x-y) / t ; t) / t \quad .
$$

(1) implies that $D$ must in general depend on $y$, and the $D(x, t ; y)$ that satisfies (1) (assuming existence) represents the effective $D$ for an initial delta-distribution of solute at $y$. Now, suppose $D^{\star}(x, t)$ satisfys

$$
\frac{\partial P^{\star}}{\partial t}+u(t) \frac{\partial P^{\star}}{\partial x}=\frac{\partial}{\partial x}\left(D^{\star} \frac{\partial P^{\star}}{\partial x}\right)
$$

Then

$$
D(x, t ; y)=D^{\star}(x-y, t)
$$

$D^{\star}$ is obtained by direct integration of (2):

$$
D^{*}(x, t)=\frac{\int_{-\infty}^{x} a(y, t) d y+m(t)}{b(x, t)}
$$

A. 1 
where $m(t)$ is arbitrary and

$$
\begin{aligned}
& t^{2} a(x, t)=-P(V ; t)-V P_{V}(V ; t)+u(t) P_{V}(V ; t)+t P_{t}(V: t) \\
& t^{2} b(x, t)=P_{V}(V ; t)
\end{aligned}
$$

with $V=x /$ t. Using

$$
\int_{-\infty}^{x} a(y, t) d y=\int_{-\infty}^{x / t} a(t v, t) d(t V)
$$

and integration by parts gives

$$
D^{*}(x, t)=\left[-t(V-u(t)) P(V ; t)+t^{2} \int_{-\infty}^{V} P_{t}(V ; t) d V+m(t)\right] / P(V ; t)
$$

with $V=x / t$.

Eq. 4 is an effective dispersion function only if it is positive when $u(t)$ is the mean $\bar{V}(t)$. 
Now when Eq. 4 is independent of $x$ and positive, the effective dispersion defined by Eq. 3 is also independent of $y$. Suppose that the velocity P.d.f. is independent of $t$, and let $m=0$. Then

$$
D *(V t, t)=-t(V-u(t)) P(V) / P^{\prime}(V)
$$

with $P^{\prime}=d P / d V$.

Eq. 5 defines an effective dispersion function for Eq. 1 independent of $y$ if it is positive and independent of V. The Gaussian velocity P.d.f.

$$
P(V)=\frac{1}{\sqrt{2 \pi \mu}} e^{-(V-u)^{2} / 2 \mu^{2}}
$$

with constant $u$ and $\mu$ satisfys the required conditions, and $D=\mu^{2} t$ follows. The p.d.f. satisfying Eq. 2 is then a Gaussian

$$
P *(x, t)=\frac{1}{\sqrt{2 \pi} \sigma} e^{-(x-u t)^{2} / 2 \sigma^{2}}
$$

with $\sigma=\mu t$.

The general relationship between an equivalent convective-dispersive system and the velocity P.d.f. is obtained by substitution of $x$-ut into (2) or by differentiation of Eq. 4:

$$
\frac{\partial P}{\partial t}+\frac{\partial}{\partial V}\left[\frac{(u(t)-V) P}{t}\right]=\frac{\partial}{\partial V}\left[\frac{D \star(V t, t)}{t^{2}} \frac{\partial P}{\partial V}\right] .
$$

Next suppose Eq. 7 is the solution of Eq. 2 with $\sigma^{2}=2 D t$ for constant $u$ and $D$. Then

$$
P(V ; t)=t P \star(V t, t)
$$


gives Eq. 6 with $\mu^{2}=2 D / t$.

Another demonstration using Fourler transforms is instructive. The Fourier transformed convection and convective-dispersion equations are

$$
\frac{d \Psi}{d t}+i V y \Psi=0
$$

and

$$
\frac{d \bar{\Psi}}{d t}+\left(i u y+D y^{2}\right) \bar{\Psi}=0
$$

respectively, where

$$
\Psi(y, t ; V)=\frac{1}{\sqrt{2 \pi}} \int_{-\infty}^{\infty} C(x, t ; V) e^{-i y x} d x
$$

and similarly for $\bar{\Psi}(y, t)$. Because the velocity p.d.f. is assumed independent of $x$, it must satisfy

$$
\bar{\Psi}(y, t)=\int \Psi(y, t ; V) P(V ; t) d V
$$

Solving Equations 9 and 10 and substituting in Eq. 11 gives

$$
e^{-\left(i u y+D y^{2}\right) t}=\int e^{-i V t y} P(V ; t) d V,
$$

since transformed initial concentrations are identifical. Equation 12 shows that the characteristic function of the velocity p.d.f. is that of a Gaussian (Eq. 6) with mean $u$ and variance $\mu^{2}=20 / t$. That is, inverting the Fourier transform in Eq. 12 gives 


$$
P(V ; t)=\int_{-\infty}^{\infty} \frac{e^{-\left(i u y t+\mu^{2}(y t)^{2} / 2\right)}}{2 \pi} e^{i V y t} d(y t),
$$

which, by using

$$
\int_{-\infty}^{\infty} e^{-a y^{2}} \frac{e^{i y x}}{\sqrt{2 \pi}} d y=\frac{e^{-x^{2} / 4 a}}{\sqrt{2 a}}, \quad(a>0)
$$

gives Eq. 6 .

NON-EXISTENCE OF AN EFFECTIVE DISPERSION

Consider a uniform velocity distribution:

$$
\begin{aligned}
P(V) & =\frac{1}{2 a}[\theta(V-\bar{V}+a)-\theta(V-\bar{V}-a)] \\
& = \begin{cases}1 / 2 \text { a when }-a<V-\bar{V}<a, \quad(a>0) \\
0 & \text { otherwise }\end{cases}
\end{aligned}
$$

The expected concentration is:

$$
\bar{c}(x, t)=\int_{\bar{V}-a}^{\bar{V}+a} c_{0}(x-V t) \frac{d V}{2 a} .
$$

Suppose

$$
C_{0}(x)=\delta(x) \text {. }
$$

Then

$$
\begin{aligned}
\tau(x, t) & =P(x / t) / t \\
& =\frac{1}{2 a t}[\theta(x / t-\bar{V}+a)-\theta(x / t-\bar{V}-a)]
\end{aligned}
$$


Calculating derivatives using $\theta^{\prime}(x)=\delta(x)$ gives

$$
\begin{aligned}
& \frac{\partial \bar{C}}{\partial x}=\frac{1}{2 a t^{2}}[\delta(x / t-\bar{V}+a)-\delta(x / t-\bar{V}-a)] \\
& \frac{\partial \bar{C}}{\partial t}=\frac{-\bar{C}(x, t)}{t}-(x / t) \frac{\partial \bar{C}}{\partial x} .
\end{aligned}
$$

Thus

$$
\frac{\partial \vec{C}}{\partial t}+\frac{\partial}{\partial x}\left(\frac{x}{t} \bar{C}\right)=0
$$

Equation 13 determines an equivalent system with $u=x / t$ and $D=0 . u$ does not equal $\bar{V}$. An effective $D$ must satisfy

$$
\overline{V C}-D \frac{\partial \bar{C}}{\partial x}=\frac{x}{t} \bar{C}
$$

or

$$
D=(\bar{V}-x / t) \bar{C} / \partial \bar{C} / \partial x
$$

But $\partial \bar{C} / \partial x=0$ for all $x$ except $x / t=\bar{V} \pm a$. Therefore (14) does not define an effective dispersion coefficient. 


\section{APPENDIX B \\ EQUIVALENT CONSTANT VELOCITY ENSEMBLES FOR \\ BOUNDARY VALUE FORMULATIONS}

Some equivalent constant velocity ensembles corresponding to constant $u$ and $D$ are obtained here. A boundary value formulation requires the initial condition

$$
\bar{C}(x, 0)=0 \quad(x>0) \quad
$$

Laplace transforming the convective-dispersion equation gives

$$
-D \frac{d^{2} \bar{\Psi}}{d x^{2}}+u \frac{d \bar{\Psi}}{d x}+s \bar{\Psi}=0
$$

where

$$
\bar{\Psi}(x, s)=\int_{0}^{\infty} \bar{C}(x, t) e^{-s t} d t \quad .
$$

Solution of Eq. 2 is:

$$
\bar{\psi}(x, s)=A_{1} e^{r_{1}(s) x}+A_{2} e^{r_{2}(s) x}
$$

with the distinct roots

$$
r(s)=u / 2 D \pm \sqrt{(u / 2 D)^{2}+s / D} \text {. }
$$


Now $\bar{C}(x, t) \rightarrow 0$ as $x \rightarrow \infty$ as requires $\bar{\Psi}(x, t) \rightarrow 0$ as $x \rightarrow \infty$. And the solution (3) becomes

$$
\bar{\Psi}(x, s)=A(s) e^{r(s) x}
$$

with

$$
r(s)=u / s D-\sqrt{(u / 2 D)^{2}+s / D}
$$

Case 1. Concentration is specified:

$$
\bar{C}(0, t)=f(t) \quad(t>0)
$$

Define

$$
h(s)=\int_{0}^{\infty} f(t) e^{-s t} d t
$$

Then

$$
A(s)=h(s)
$$

$\mathfrak{f}^{-1}$ denotes the inverse Laplace transform. Let

$$
P \star(t ; x)=\mathcal{E}^{-1}\left[e^{r(s) x}\right]
$$

Using

$$
f^{-1}\left[e^{-a \sqrt{s}}\right]=\frac{a}{2 \sqrt{\pi t^{3}}} e^{-a^{2} / 4 t} \quad(a>0)
$$


gives

$$
\begin{aligned}
& p *(t ; x)=\frac{x}{2 \sqrt{\pi D t^{3}}} e^{-(x-u t)^{2} / 4 D t} \\
= & \frac{x}{t} g(x, t) .
\end{aligned}
$$

By the Convolution theorem, the solution is given by

$$
\bar{C}(x, t)=\int_{0}^{t} f(t-\gamma) p *(\gamma ; x) d \gamma .
$$

Direct integration verifies that Eq. 6 is a travel time p.d.f.:

$$
\int_{0}^{\infty} P^{*}(\gamma ; x) d \gamma=\int_{0}^{\infty} P(V ; x) d V=\int_{0}^{\infty} q(u ; x) d u=1
$$

where

$$
q(u ; x)=\frac{2 e^{2 b}}{\sqrt{\pi}} e^{-u^{2}}-b^{2} / u^{2}
$$

and

$$
P(V ; x)=\frac{1}{\sqrt{\pi \mathrm{a} V}} e^{-(V-u)^{2} / \mathrm{a} V}
$$

with $a=4 D / x$ and $b=u / a$.

The representation of Eq. 7 with Eq. 6 given by

$$
\bar{c}(x, t)=\int_{0}^{\infty} f\left(t-x^{2} / 4 D u^{2}\right) q(u ; x) d u
$$


shows that the boundary condition is fulfilled, since $q(u ; 0)$ is a non-vanishing p.d.f. in $u$.

The moment generating function for (6) is:

$$
\begin{aligned}
M(s ; x) & =\int_{0}^{\infty} e^{-s \gamma} p \star(\gamma ; x) d \gamma \\
& =e^{r(s) x} .
\end{aligned}
$$

Another travel time p.d.f. is

$$
\begin{aligned}
P \star(t ; x) & =\frac{1}{\sqrt{\pi b t}} e^{-(t-x / u)^{2} / b t} \\
& =u g(x, t)
\end{aligned}
$$

with $b=4 D / u^{2}$, since it has the form of (8).

And the associated velcotiy p.d.f. is

$$
P(V ; x)=\frac{u}{\sqrt{\pi a V^{3}}} e^{-(V-u)^{2} / a V}
$$

with $a=4 D / x$.

Fickian flux is:

$$
\bar{F}(x, t)=u \bar{C}(x, t)-D \frac{\partial \bar{C}}{\partial x}(x, t) .
$$


Direct calculation of Eq. 1 for Eq. 6 ylelds

$$
\begin{aligned}
F(x, t) & =\int_{0}^{\infty} f(t-r)\left[u \frac{x}{r} g(x, r)-D \frac{\partial}{\partial x}\left(\frac{x}{r} g(x, r)\right)\right] d \gamma \\
& =\int_{0}^{\infty} f(t-r)\left[\frac{u+x / \gamma}{2}-\frac{D}{x}\right] g(x, r) \frac{x}{r} d r
\end{aligned}
$$

Therefore

$$
\bar{F}(x, t)=\int_{0}^{\infty} F(x, t ; \gamma) P *(r ; x) d r
$$

where the stochastic flux for this case is

$$
F(x, t ; \gamma)=f(t-\gamma)\left[\frac{u+x / \gamma}{2}-\frac{D}{x}\right] .
$$

But Eq. 13 does not equal the convective stochastic flux $U(x, t ; x / r)$ defined by

$$
U(x, t ; V)=V f(t-x / V)
$$

Now let

$$
\bar{F}(x, t)=\int_{0}^{\infty} U(x, t ; x / r) G(x, y) d y
$$

with

$$
G(x, y)=\left[\frac{u+x / y}{2}-\frac{D}{x}\right] g(x, y)
$$

B.5 
Now

$$
\int_{0}^{\infty} G(x, r) d r=1-D / u x
$$

shows that Eq. 15 cannot define a p.d.f. in $\gamma$. Therefore, the flux does not have an equivalent ensemble for Eq. 14.

Case 2. The flux is specified by:

$$
F(0, t)=J(t) \quad(t>0)
$$

in one of two ways,

$$
\begin{aligned}
\text { (i) } J(t) & =u f(t) \\
\text { (ii) } J(t) & =f(t) .
\end{aligned}
$$

The Fickian flux (time independent $u$ and $D$ ) satisfies

$$
\frac{\partial \bar{F}}{\partial t}+u \frac{\partial \bar{F}}{\partial x}=D \frac{\partial^{2}}{\partial x^{2}} \bar{F}
$$

because it satisfies the conservation equations:

$$
\frac{\partial \bar{C}}{\partial t}+\frac{\partial \bar{F}}{\partial x}=0
$$

Therefore the solution of Eq. 17 subject to Eq. 16 and $\bar{F}(x, 0)=0$ ix

$$
\bar{F}(x, t)=\int_{0}^{t} J(t-r) p \star(r ; x) d r
$$

with the travel time p.d.f. (Eq. 6). 
Laplace transforming the flux (Eq. 11) and the boundary condition (Eq. 16) determines Eq. 4 with

$$
A(s)=h(s) /(u-\operatorname{Dr}(s))
$$

where $h$ is the transform of $J$. Concentration is then given by

$$
\bar{C}(x, t)=\int_{0}^{t} J(t-\gamma) G(x, \gamma) d \gamma
$$

where

$$
G(x, t)=\Sigma^{-1} \quad\left[e^{r(s) x} /(u-\operatorname{Dr}(s)]\right.
$$

Inverting this Laplace transform by using tables gives

$$
G(x, t)=\frac{1}{\sqrt{\pi D t}} \exp \left(-\frac{x^{2}}{4 D t}-t D a^{2}+a x\right)-a e^{2 a x} \operatorname{erfc}\left(a \sqrt{D t}+\frac{x}{2 \sqrt{D t}}\right)
$$

with $\mathrm{a}=u / 2 \mathrm{D}$. It is found that

$$
G(x, t)=2 g(x, t)-\frac{u}{D} \int_{0}^{\infty} e^{-y u / D} g(x+y, t) d y
$$

or alternatively,

$$
G(x, t)=\frac{e^{x u / D}}{D t} \int_{x}^{\infty} e^{-y u / D} y g(y, t) d y
$$


Equation 23 can be obtained also by assuming Eq. 21 and solving for $G$ satisfying

$$
u G(x, t)-D \frac{\partial G}{\partial x}(x, t)=\frac{x}{t} g(x, t),
$$

in view of Eq. 11 and Eq. 19. Properties of Eq. 23 are:

$$
G(x, t) \geq 0
$$

for $u>0$ and $x, t \geq 0$, and

$$
\int_{0}^{\infty} G(x, \gamma) d \gamma=1 / u
$$

Equivalent ensembles for the concentration must satisfy

$$
\tau(x, t)=\int_{0}^{\infty} C(x, t ; x y) P *(r, x) d \gamma
$$

for

$$
\begin{aligned}
& \text { (i) } \quad c(x, t ; V)=f(t-x / V) \\
& \text { (ii) } \quad c(x, t ; V)=f(t-x / V) / V .
\end{aligned}
$$

Then

$$
\begin{aligned}
& \text { (i) } \quad p *(\gamma ; x)=u G(x, \gamma) \\
& \text { (ii) } \quad p *(\gamma ; x)=\frac{x}{\gamma} G(x, \gamma)
\end{aligned}
$$


define the travel time distributions. (i) determines a p.d.f. given by

$$
P^{*}(r ; x)=2 P_{0}^{*}(r ; x)-\frac{u}{D} \int_{0}^{\infty} e^{-y u / D} P_{0}^{*}(r ; x+y) d y
$$

where

$$
P_{0}(r ; x)=u g(x, r)
$$

(ii) gives

$$
P^{*}(r ; x)=2 P_{0}^{*}(r ; x)-\frac{U}{D} \int_{0}^{\infty} e^{-y u / D} \frac{x}{x+y} P_{0}^{*}(r ; x+y) d y
$$

where

$$
P_{0}^{*}(r ; x)=\frac{x}{r} g(x, r)
$$

Integrating Eq. 28 gives

$$
\int_{0}^{\infty} P *(r ; x) d y=2-\frac{u}{D} \int_{0}^{\infty} e^{-y u / D} \frac{x}{x+y} d y>1
$$

since

$$
2-\frac{u}{D} \int_{0}^{\infty} e^{-y u / D} d y=1
$$

and $x /(x+y)<1$.

B.9 
Therefore Eq. 25 (ii) does not define a p.d.f. for $r>0$. Equation 26 has an interesting property: $p^{*}=p_{0}^{*}$ for $x=u t$. Note that for Eq. 21 with (ii), we have that

$$
\bar{C}(x, t)=\int_{0}^{\infty} \frac{f(t-r)_{p \star}(r ; x) d r}{u}
$$

with p.d.f. Eq. 26 defines an ensemble expectation of a stochastic concentration $f(t-x / V) / u$ not satisfying the same boundary condition as in Eq. 24 (ii).

Equivalent ensembles for the Fickian flux must satisfy

$$
F(x, t)=\int_{0}^{\infty} U(x, t ; x / y) P_{0}^{*}(r ; x) d y
$$

for

$$
\begin{aligned}
& U(x, t ; V)=V f(t-x / V) \\
& U(x, t ; V)=f(t-x / V) .
\end{aligned}
$$

Then the travel time P.d.f. given by (27) for (i) and (29) for (i1) determine the equivalent ensembles. Thus, it is shown that the equivalent ensembles for concentration and flux may not have identical P.d.f. for the travel time. The moment generating function of (26) is:

$$
M(S ; x)=\frac{u e^{r(s) x}}{u-\operatorname{Dr}(s)}
$$




\section{APPENDIX C}

\section{ELEMENTARY SOLUTIONS IN ONE-DIMENSION}

Some solutions are found for the convection equation:

$$
\frac{\partial C}{\partial t}+V(x, t) \frac{\partial C}{\partial x}=0
$$

Position independent case, $V(t)$ :

let:

$$
C(x, t)=Z(x, y)
$$

where:

$$
y=\int_{0}^{t} V(\gamma) d \gamma
$$

(1) is transformed by substitution of (2) into:

$$
\frac{\partial Z}{\partial x}+\frac{\partial Z}{\partial y}=0
$$

Time independent case, $V(x)$ :

let:

$$
c(x, t)=z(t, \gamma)
$$

where:

$$
\gamma=\int_{0}^{x} \mathrm{~d} \xi / V(\xi)
$$


(1) is transformed by substitution of (4) into:

$$
\frac{\partial Z}{\partial t}+\frac{\partial Z}{\partial Y}=0
$$

We need only solve an equation of the form (3) or (5).

The characteristic equations for (3) are:

$$
\frac{d x}{d \xi}=1, \frac{d y}{d \xi}=1, \frac{d z}{d \xi}=0
$$

(6) are solved for Cauchy-type boundary conditions (initial conditions at $\xi=0$ ) given by $x=x_{0}(\zeta), z=z_{0}(\zeta), p=p_{0}(\zeta), q=q_{0}(\zeta)$ with $(p=\partial z / \partial x$ and $q=\partial z / a y)$

$$
p_{0}+q_{0}=0 \text { and } \frac{d z_{0}}{d \xi}=p_{0} \frac{d x_{0}}{d \xi}+q_{0} \frac{d y_{0}}{d \xi}
$$

to yield a solution of $(3)$ :

$$
x=\xi+x_{0}(\zeta), y=\xi+y_{0}(\zeta), z=z_{0}(\zeta)
$$

(7) determines w such that:

$$
x-y=x_{0}(\zeta)-y_{0}(\zeta)
$$

and:

$$
w(x-y)=\zeta
$$

then:

$$
z=z_{0}[w(x-y)]
$$

is the particular solution. Note that: 


$$
\begin{aligned}
& \left.\frac{\partial Z}{\partial x}\right)_{\xi=0}=Z_{0}^{\prime}(\zeta) w^{\prime}\left[x_{0}(\zeta)-y_{0}(\zeta)\right]=P_{0}(\zeta) \\
& \left.\frac{\partial z}{\partial x}\right)_{\xi=0}=Z_{0}^{\prime}(5) w^{\prime}\left[x_{0}(\zeta)-y_{0}(5)\right]=q_{0}(5)
\end{aligned}
$$

is requitred.

(9) demonstrates that all solutions of (1) for either position or time independent velocity are of the form:

$$
C(x, t)=f\left[x-\int_{0}^{t} V(y) d Y\right]
$$

or

$$
C(x, t)=f\left[t-\int_{0}^{x} d \xi / V(\xi)\right]
$$

respectively, where $f$ is determined by the specific initial and boundary conditions.

Initial Value Formulation

$$
C(x, 0)=C_{0}(x)
$$

is specified at $t=0$. Solutions are:

$$
C(x, t)=C_{0}\left[x-\int_{0}^{t} V(Y) d Y\right]
$$

and

$$
C(x, t)=C_{0}\left[T^{-1}[T(x)-t]\right]
$$


where:

$$
T(x)=\int_{0}^{x} d \xi / V(\xi)
$$

Boundary Value Formulation

$$
C(0, t)=f(t)
$$

is specified at $x=0$.

Solutions are:

$$
C(x, t)=f\left[L^{-1}\left[L^{\prime}(t)-x\right]\right]
$$

where:

$$
L(t)=\int_{0}^{t} V(\gamma) d \gamma
$$

and:

$$
C(x, t)=f\left[t-\int_{0}^{x} d \xi / V(\xi)\right]
$$

Boundary conditions for (13) and (14) are:

$$
C(x= \pm+\infty, t)=0,(t \geq 0) .
$$

Initial conditions for (16) and (17) are: -

$$
c(0, x)=0, \quad(x \geq 0)
$$


Solutions of Conservation Equation

$$
\frac{\partial \rho}{\partial t}+\frac{\partial}{\partial x}\left(V_{\rho}\right)=0
$$

Define convective flux:

$$
U(x, t)=V(x) \rho(x, t)
$$

Time independent velocity is assumed. Then $U$ satisfies:

$$
\frac{\partial U}{\partial t}+V(x) \frac{\partial U}{\partial x}=0
$$

Solutions of (20) are like those of (1).

Initial Value Formulation

$$
U(x, 0)=U_{0}(x) \equiv V(x) \rho_{0}(x)
$$

Solution for (21) is:

$$
\rho(x, t)=V\left[T^{-1}[T(x)-t]\right] \rho_{0}\left[T^{-1}[T(x)-t]\right] / V(x)
$$

Boundary Value Formulation

$$
U(0, t)=J(t)=V_{0} f(t)
$$

Solution for (23) is:

$$
\rho(x, t)=\frac{V_{0}}{V(x)} f[t-T(x)]
$$

where $T(x)$ is the travel time defined in (14). 


\section{An Integral Transform Method}

Define the Laplace transform with respect to time:

$$
\Psi(x, s)=\int_{0}^{\infty} d t e^{-s t} c(x, t)
$$

Velocity $V(x)$ is assumed time independent. Suppose that initial and boundary conditions are specified by:

$$
\begin{aligned}
& C(x, t=0)=C_{0}(x) \\
& C\left(x_{0}, t\right)=f(t)
\end{aligned}
$$

where $C_{0} \equiv 0$ if $x<x_{0}$ and $f(t) \equiv 0$ if $t<0$. Moreover require:

$$
C_{0} \geq 0 \text { and } \int_{-\infty}^{\infty} C_{0}(x) d x<\infty
$$

The Laplace transformed convection Eq. 1 is:

$$
V(x) \frac{d \psi}{d x}(x, s)+s \psi(x, s)=C_{0}(x)
$$

Solution of (27) is:

$$
\psi(x, s)=e^{-s T(x)}\left\{\int_{x_{0}}^{x} \frac{C_{0}(\xi)}{V(\xi)} e^{s T(\xi)} d \xi+g(s)\right\}
$$

where:

$$
g(s)=\Sigma[f ; s]
$$

and: 


$$
T(x)=T\left(x_{;} ; x_{0}\right)=\int_{x_{0}}^{x} d \xi / V(\xi)
$$

Define $\delta_{+}$such that:

$$
\int_{0}^{\infty} \delta_{+}(t-a) e^{-s t} d t=\left\{\begin{array}{lll}
e^{-a s} & \text { if } & a>0 \\
0 & \text { if } & a<0
\end{array}\right.
$$

Taking the inverse Laplace transform of (28) and using the convolution theorem gives:

$$
\begin{aligned}
C(x, t) & =\int_{x_{0}}^{x} \frac{C_{0(\xi)}}{V(\xi)} s_{+}[t-T(x ; \xi)] d \xi \\
& +\int_{0}^{t} f(t-\gamma) \delta_{+}[\gamma-T(x)] d \gamma
\end{aligned}
$$

now:

$$
\begin{aligned}
\int_{0}^{t} f(t-\gamma) \delta_{+}[\gamma-T(x)] d \gamma & =\theta[T(x)] \theta[t-T(x)] f[t-T(x)] \\
& =f[t-T(x)]
\end{aligned}
$$

by requiring $V(x) \geq 0$. Assuming the inverse $T^{-1}$ exists, which is true for $v>0$ : 


$$
\begin{aligned}
\int_{x_{0}}^{x} \frac{C_{0}(\xi)}{V(\xi)} \delta_{+}[t-T(x ; \xi)] d \xi & =\int_{x_{0}}^{x} C_{0}(\xi) \delta_{+}[T(\xi)-T(x)+t] d T(\xi) \\
& =\int_{0}^{T(x)} C_{0}\left[T^{-1}(\gamma)\right] \delta_{+}[\gamma-T(x)+t] d \gamma \\
& =\theta[T(x)-t] C_{0}\left[T^{-1}[T(x)-t]\right]
\end{aligned}
$$

The solution to (29) is:

$$
C(x, t)=\theta[T(x)-t] C_{0}\left[T^{-1}[T(x)-t]\right]+f[t-T(x)]
$$

for:

$$
x \geq x_{0}, t \geq 0
$$

The initial condition formulation of Equation 30 has $f \equiv 0$, and the boundary condition formulation has $C_{0} \equiv 0$. Notice that the concentration solution domain for the initial condition formulation is $x_{2} x_{0}$ and $0 \leq t \leq T(x)$.

Another extended solution is obtainable by using a Fourier transform. Let

$$
\psi(x, a)=\frac{1}{\sqrt{2 \pi}} \int_{-\infty}^{\infty} C(x, t) e^{-l a t} d t .
$$

The Fourier transformed Equation 1 is:

$$
\operatorname{ta} \psi(x, \alpha)+v(x) \frac{d \psi}{d x}=0
$$

by requiring $C(x, t=+\infty)=0$. And the solution is:

$$
\psi(x, \alpha)=h(\alpha) e^{-i a t(x)},
$$


where $h(a)$ is the Fourier transform of $f(t)$. Inverting the transform of Equation 32 gives:

$$
\begin{aligned}
C(x, t) & =\int_{-\infty}^{\infty} f(t-y) \delta[y-T(x)] d y \\
& =f[t-T(x)]
\end{aligned}
$$

No restrictions on velocity direction are required here. An extended solution for the initial value formulation is given by

$$
\begin{aligned}
C(x, t) & =\int_{-\infty}^{\infty} \frac{C_{0}(\xi)}{V(\xi)} \delta[t-T(x ; \xi)] d \xi \\
& =\int_{-\infty}^{\infty} C_{0}(\xi) \delta[T(\xi)-T(x)+t] d T(\xi),
\end{aligned}
$$

which yields Equation 14 when the required inverse $T^{-1}$ exists. 


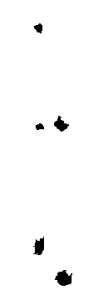

.

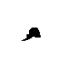

$?$

$\forall$

$\forall$

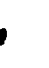

. 


\section{DISTRIBUTION}

No. of

Copies

OFFSITE

\section{A. A. Churm}

DOE Chicago Patent Group

9800 South Cass Avenue

Argonne, IL 60439

27 DOE Technical Information Center

2 Argonne National Laboratory Reference Library 9800 South Cass Avenue Argonne, IL 60439

20 Battelle Memorial Institute Office of Nuclear Waste Isolation

Attn: Beverly Rawles

505 King Avenue

Columbus, $\mathrm{OH} 43201$

Albin Brandstetter

Office of Nuclear Waste Isolation

505 King Avenue

Columbus, $\mathrm{OH} 43201$

John Bredehoeft

U.S. Geological Survey

Reston, VA 22092

John Bird

Geology Department

Corne 11 University

Ithaca, NY 14853

Harry C. Burkholder

Office of Nuclear Waste Isolation

505 King Avenue

Columbus, $\mathrm{OH} 43201$
No. of

Copies

2 Brookhaven National Laboratory

Reference Section

Information Division

Upton, Long Is land, NY 11973

Wayne A. Carbiener

Office of Nuclear Waste

Isolation

Battelle Memorial Institute

$505 \mathrm{King}$ Avenue

Columbus, $\mathrm{OH} 43201$

Neville G. W. Cook

Dept of Materials Science and Mineral Engineering

Hearst Mining Building

University of California

Berkeley, CA 94720

Carl R. Cooley

DOE Office of Nuclear Waste Management

Washington, DC 20545

Mike Cullingford

Probablistic Analys is Staff

Nuclear Regulatory Commission

Mail Stop 3103

Washington, DC 20555

Dan Egan

Environmental Protection Agency

Office of Radiation Programs

Technology Assessment Division

M/S AW559

Washington, DC 20460

Robert M. Garrells

Dept of Geologic Sciences

Northwestern University

Evanston, IL 60201 
No. of

Copies

Lynn W. Gelhar

New Mexico Institute of Mining and Technology

Socorro, New Mexico 89801

Mark A. Harwe 11

P.0. Box 667

Cannon Beach, OR 97110

Colin A. Heath

DOE Office of Nuclear Waste Management

Washington, DC 20545

William M. Hewitt

Office of Nuclear Waste Isolation

Battelle Memorial Institute

505 King Avenue

Columbus, $\mathrm{OH} \quad 43201$

Peter L. Hofmann

Office of Nuclear Waste Isolation

Battelle Memorial Institute

505 King Avenue

Columbus, $\mathrm{OH} 43201$

Muzaffer Kehnemuyi

Office of Nuclear Waste I solation

Battelle Memorial Institute

505 King Avenue

Columbus, $\mathrm{OH} 43201$

John F. Kircher

Office of Nuclear Waste Isolation

Battelle Memorial Institute

505 King Avenue

Columbus, $\mathrm{OH} 43201$

Ron B. Lantz

INTERA Environmental

Consultants, Inc.

11511 Katy Freeway, Suite 630

Houston, TX 77079
No. of

Copies

2 Lawrence Berkeley Laboratory

Reference Library

University of California

Berkeley, CA 94720

Stan E. Logan

Los Alamos Technical

Associates, Inc.

P.0. Box 410, 1650 Trinity Drive

Los Alamos, NM 87544

2 Lawrence Livermore Laboratory

Reference Library

P.0. Box 808

Livermore, CA 94550

2 Los Alamos Scientific Laboratory

Reference Library

P.0. Box 1663

Los Alamos, NM 87544

J. B. Martin

Asst. Director for Radioactive Waste Management Branch

NRC Division of Materials and Fuel Cycle Facility Licensing

Washington, DC 20555

John T. McGinnis

Office of Nuclear Waste Isolation

Battelle Memorial Institute

505 King Avenue

Columbus, $\mathrm{OH} 43201$

James W. Mercer

Geotrans, Inc.

201 Elden St.

Herndon, Virginia 22070

2 Barry N. Naft

NUS Corporation

4 Research Place

Rockville, MD 20805 
No. of

Copies

2 J. 0. Neff

Department of Energy

Columbus Program Office

505 King Avenue

Columbus, $\mathrm{OH} 43201$

Shlomo $P$. Neuman

Dept. Hydrology \& Water Res.

Univ. of Arizona

Tucson, Arizona 85721

Donald R. Nielsen

Land, Air, Water Resources Dept.

113 Veihmeyer

Univ. of California, Davis

Davis, California 99353

2 Neil A. Norman

Environmental Sciences

Department

Bechtel National Inc.

P.0. Box 3965

San Francisco, CA 94105

2 Oak Ridge National Laboratory

Central Research Library

Document Reference Section

Oak Ridge, TN 37830

Frank L. Parker

Dept. of Environmental Eng.

Vanderbilt University

Nashville, TN 37235

George F. Pinder

Dept. of Civil Engineering

Princeton University

Princeton, NJ 08540

H. L. Ragsdale

Department of Biology

Emory University

Atlanta, GA 30322

2 Savannah River Laboratory

Reference Library

Alken, SC 29801
No. of

Copies

L. E. Scriver

Dept. of Chemtcal Engineering

421 Washington Avenue S. E.

Untv. of Minnesota

Minneapoles, Minnesota 55455

Arthur J. Soinski

Energy Resources Conservation and Development Commission

1111 Howe Avenue

Sacramento, CA 95825

Garrison Sposito

Dept. of Soil and Environmental Sciences

Univ. of Calif. Riverside

Riverside, California 92521

Howard P. Stephens

Sandia Laboratories

P.0. Box 5800

Albuquerque, NM 87115

David B. Stewart

U.S. Geological Survey

National Center 959

Reston, VA 22092

Der-Hua Edward Tang

Intera

11999 Katy Freeway Suite 610

Houston, Texas 77079

Robert Williams

Electric Power Research Inst. 3412 Hillview Avenue

P.0. Box 10412

Palo Alto, CA 94304

Paul A. Witherspoon

Earth Sciences Division

Lawrence Berkeley Laboratory

Building 90

Berkeley, CA 94720 
5 DOE Richland Operations Office

0. J. Elgert

R. B. Goranson

H. E. Ransom

J. J. Schretber

F. R. Standerfer

8 Rockwell Hanford Operations
R. G. Baca
R. L. Bielefeld
D. J. Carre 11
R. A. Deju
F. A. Deluca
R. E. Gephart
L. S. Leonhart
Rockwe 11 Document Control

89 Paciffc Northwest Laboratory

G. L. Benson

C. R. Cole

W. J. Deutsch

F. H. Dove (20)

J. W. Lindberg

M. R. Kreiter

R. W. Nelson

G. M. Petrie

A. M. Platt

R. J. Serne

D. J. Silveria

J. K. Soldat

C. S. Simmons (20)

G. W. Gee

D. Webber (20)

Technical Information Library (5)

Publishing Coordination (2)

Water and Land Resources Dept. Library (10) 